\title{
A COMPARISON OF PALEY-WIENER THEOREMS FOR REAL REDUCTIVE LIE GROUPS
}

\author{
E. P. VAN DEN BAN, S. SOUAIFI
}

\section{Contents}

Introduction

1. Notation and preliminaries

2. Holomorphic families of representations and their derivatives

2.1. The derivation process

2.2. Holomorphic families of continuous representations

2.3. Holomorphic families of admissible $(\mathfrak{g}, K)$-modules

2.4. Parabolic induction

3. The Arthur-Campoli relations

3.1. The Arthur-Campoli relations

3.2. Reformulation of the Arthur-Campoli relations

4. Delorme's interwining conditions

4.1. Successive derivatives

4.2. The intertwining conditions

4.3. A simplification of the intertwining conditions

4.4. Reduction to a single minimal parabolic subgroup

4.5. Reformulation in our setting

5. Conditions in terms of the Hecke algebra

5.1. Some general facts on approximately unital $A$-modules

5.2. A double commutant theorem

5.3. Application to Harish-Chandra modules

5.4. Proof of the main theorem

5.5. Another useful characterization of the Paley-Wiener space

Appendix. Some topological results

References

\section{INTRODUCTION}

In this paper we make a detailed comparison between the Paley-Wiener theorem of J. Arthur [1], and the one recently established by P. Delorme [5].

Let $G$ be a real reductive Lie group of the Harish-Chandra class and let $K$ be a maximal compact subgroup. Let $C_{c}^{\infty}(G, K)$ denote the space of smooth compactly supported functions on $G$ which behave finitely under both left and right translation by $K$. The Paley-Wiener theorem of each of the above mentioned authors describes the image of $C_{c}^{\infty}(G, K)$ under Fourier transformation, in terms of a so called Paley-Wiener space. In this paper we will show that the two Paley-Wiener spaces are equal, without using the 
proof or the validity of any of the associated Paley-Wiener theorems. It thus follows that the two theorems are equivalent from an a priori point of view.

In order to be able to be more specific about the contents of this paper, we shall first give a more detailed description of the two Paley-Wiener theorems mentioned above.

Let $\mathfrak{g}=\mathfrak{k} \oplus \mathfrak{p}$ be a Cartan decomposition associated with the maximal compact subgroup $K$. Here and in the following we use the convention to denote Lie groups by Roman capitals, and their Lie algebras by the corresponding lower case German letters. Let $\mathfrak{a}$ be a maximal abelian subspace of $\mathfrak{p}$, and let $A=\exp \mathfrak{a}$ be the associated vectorial closed subgroup of $G$. We denote by $\mathcal{P}(A)$ the (finite) set of cuspidal parabolic subgroups of $G$ containing $A$. Each parabolic subgroup $P \in \mathcal{P}(A)$ has a Langlands decomposition of the form $P=M_{P} A_{P} N_{P}$, with $A_{P} \subset A$. Let $M_{P, d s}^{\wedge}$ denote the set of (equivalence classes of ) discrete series representations of $M_{P}$. For $(\xi, \mathcal{H}) \in M_{P, d s}^{\wedge}$ and $\lambda \in \mathfrak{a}_{P \mathbb{C}}^{*}$, we denote by $\pi_{\xi, \lambda}=\pi_{P, \xi, \lambda}$ the representation of $G$ induced from the representation $\xi \otimes\left(\lambda+\rho_{P}\right) \otimes 1$ of $P$. The associated module of smooth vectors for $\pi_{\xi, \lambda}$ has a realization on the space $C^{\infty}(K: \xi)$ consisting of smooth functions $\psi: K \rightarrow \mathcal{H}_{\xi}$, transforming according to the rule:

$$
\psi(m k)=\xi(m) f(k), \quad \text { for all } \quad k \in K, m \in K \cap M_{P} .
$$

Accordingly, each function $f \in C_{c}^{\infty}(G)$ has an operator valued Fourier transform

$$
\hat{f}(P, \xi, \lambda):=\pi_{\xi, \lambda}(f)=\int_{G} f(x) \pi_{\xi, \lambda}(x) d x \in \operatorname{End}\left(C^{\infty}(K: \xi)\right) .
$$

Moreover, the endomorphism $\hat{f}(P, \xi, \lambda)$ depends holomorphically on the variable $\lambda \in \mathfrak{a}_{P \mathbb{C}}^{*}$. If $f$ is bi-K-finite, then $\hat{f}(P, \xi, \lambda)$ belongs to the space $\mathcal{S}(P: \xi)$ of bi-K-finite elements of $\operatorname{End}\left(C^{\infty}(K: \xi)\right)$. Moreover, the holomorphic maps $\lambda \mapsto \hat{f}(P, \xi, \lambda)$ are non-zero for only finitely many of the pairs $(P, \xi)$. It follows that $\hat{f}(P)$ may be viewed as an element of the algebraic direct sum

$$
\oplus_{\xi} \quad \mathcal{O}\left(\mathfrak{a}_{P \mathbb{C}}^{*}\right) \otimes \mathcal{S}(P: \xi) .
$$

We define the pre-Paley-Wiener space $\mathrm{PW}_{P}^{\mathrm{pre}}(G, K)$ as the space of elements $\left(\varphi(P, \xi) \mid \xi \in M_{P, d s}^{\wedge}\right)$ in (0.1) for which there exists a constant $R>0$ and for every $n>0$ a constant $C_{n}>0$ such that

$$
\|\varphi(P, \xi, \lambda)\| \leq C_{n}(1+|\lambda|)^{-n} e^{R|\operatorname{Re} \lambda|}
$$

for all $\xi, \lambda$.

We can now describe the Paley-Wiener space involved in Arthur's theorem in [1]. Let $P_{0}$ be a fixed minimal parabolic subgroup in $\mathcal{P}(A)$. Its Langlands decomposition is of the form $P_{0}=M A N_{0}$, where $M$ is the centralizer of $A$ in $K$. The Arthur Paley-Wiener space

$$
\mathrm{PW}^{A}(G, K)
$$

is defined as the space of $\varphi \in \mathrm{PW}_{P_{0}}^{\mathrm{pre}}(G, K)$ satisfying all finite linear relations of the form

$$
\sum_{i}\left\langle\varphi\left(P_{0}, \xi_{i}, \lambda_{i} ; u_{i}\right), \psi_{i}\right\rangle=0
$$


with $\xi_{i} \in M^{\wedge}, \psi_{i} \in \mathcal{S}\left(P_{0}: \xi_{i}\right)_{K \times K}^{*}, \lambda_{i} \in \mathfrak{a}_{\mathbb{C}}^{*}$ and with $u_{i} \in S\left(\mathfrak{a}^{*}\right)$ acting as differential operators in the $\lambda$-variable (see Section 2.1 for notation), as soon as these relations are satisfied by all families of functions $\left(\lambda \mapsto \pi_{P_{0}, \xi, \lambda}(x) \mid\right.$ $\xi \in M^{\wedge}$ ), for $x \in G$. These are the so-called Arthur-Campoli relations. In [1, Arthur defines a similar Paley-Wiener space involving all minimal parabolic subgroups from $\mathcal{P}(A)$. In [2] this space is shown to be isomorphic to the one defined in (0.3).

Next, let us describe the Paley-Wiener space introduced by Delorme [5]. The definition of this Paley-Wiener space involves the operation of taking successive derivatives of a family $\pi_{\lambda}$ of representations, depending holomorphically on a parameter $\lambda \in \mathfrak{a}_{P \mathbb{C}}^{*}$, for some $P \in \mathcal{P}(A)$. Such an operation is encoded by a sequence $\eta=\left(\eta_{1}, \ldots, \eta_{N}\right)$ in $\mathfrak{a}_{P \mathbb{C}}^{*}$, listing the directions in which the derivatives should be taken successively. The associated family $\pi_{\lambda}^{(\eta)}$ is again a holomorphic family of representations. The operation of derivation also applies to a holomorphic family $\lambda \mapsto \varphi_{\lambda}$ of continuous endomorphisms of $\pi_{\lambda}$ and then gives a holomorphic family $\varphi^{(\eta)}$ of endomorphisms of $\pi_{\lambda}^{(\eta)}$. Let $\mathcal{D}$ be the set of all 4-tuples $(P, \xi, \lambda, \eta)$ with $P \in \mathcal{P}(A)$, $\xi \in M_{P, d s}^{\wedge}, \lambda \in \mathfrak{a}_{P \mathbb{C}}^{*}$ and $\eta$ a finite sequence of linear functionals from $\mathfrak{a}_{P \mathbb{C}}^{*}$ as above. Given a datum $\delta=(P, \xi, \lambda, \eta)$ we define $\pi_{\delta}:=\pi_{P, \xi, \lambda}^{(\eta)}$. Moreover, given $\varphi \in \mathrm{PW}_{P_{0}}^{\mathrm{pre}}(G, K)$ we define $\varphi_{\delta}$ in a similar fashion. Finally, given a sequence $\delta=\left(\delta_{1}, \ldots, \delta_{N}\right)$ of data from $\mathcal{D}$, we write $\pi_{\delta}:=\pi_{\delta_{1}} \oplus \cdots \oplus \pi_{\delta_{N}}$, and $\varphi_{\delta}:=\varphi_{\delta_{1}} \oplus \cdots \oplus \varphi_{\delta_{N}}$.

Delorme's Paley-Wiener space is defined as the space $\mathrm{PW}^{D}(G, K)$ of functions $\varphi \in \oplus_{P \in \mathcal{P}(A)} \mathrm{PW}_{P}^{\mathrm{pre}}(G, K)$ such that

(a) for each finite sequence $\delta \in \mathcal{D}^{N}$ the function $\varphi_{\delta}$ preserves all invariant subspaces of $\pi_{\delta}$

(b) for any two finite sequences $\delta_{1} \in \mathcal{D}^{N_{1}}$ and $\delta_{2} \in \mathcal{D}^{N_{2}}$, and any two sequences of closed invariant subspaces $U_{j} \subset V_{j}$ for $\pi_{\delta_{j}},(j=1,2)$, the induced maps $\bar{\varphi}_{\delta_{j}} \in \operatorname{End}\left(V_{j} / U_{j}\right)$ are intertwined by all $G$-equivariant operators $T: V_{1} / U_{1} \rightarrow V_{2} / U_{2}$.

There is a natural map $\mathrm{PW}^{D}(G, K) \rightarrow \mathrm{PW}_{P_{0}}^{\text {pre }}(G, K)$, given by

$$
\left(\varphi(P, \xi) \mid P \in \mathcal{P}(A), \xi \in \widehat{M}_{P, d s}\right) \mapsto\left(\varphi\left(P_{0}, \xi\right) \mid \xi \in \widehat{M}_{P_{0}}\right) .
$$

In this paper we show that the map (0.5) is a linear isomorphism from $\mathrm{PW}^{D}(G, K)$ onto $\mathrm{PW}^{A}(G, K)$, see Theorem 5.11.

To understand better the conditions involving the derivatives in the definition of the Paley-Wiener spaces, we start, in Section 2, with the study of holomorphic families and their derivatives. Instead of focusing on first order derivatives, we replace a holomorphic family by the associated holomorphic section in a suitable jet bundle. This idea also occurs in W. Casselman's paper [4, §9]. We reformulate it slightly, by using a suitable trivialization of the jet bundle. Our construction starts with fixing a finite dimensional module $E$ for the ring $\mathcal{O}_{0}$ of germs of holomorphic functions (of $\lambda$ ) at zero. It then gives, for $\pi_{\lambda}$ a holomorphic family of representations in a fixed complete space $V$ a new holomorphic family $\pi_{\lambda}^{(E)}$ in the space $E \otimes V$. The differentiation procedure of Delorme turns out to be a special case of this procedure, with $E$ a suitable module of dimension 2 . In the same Section 2 we study 
how the functor $\pi_{\lambda} \mapsto \pi_{\lambda}^{(E)}$ behaves with respect to analytic families of intertwining operators and with respect to induction.

In the next section, 3, we give an equivalent definition of Arthur's PaleyWiener space by invoking the functors $\pi \mapsto \pi^{(E)}$, instead of the derivations given by elements of $S\left(\mathfrak{a}^{*}\right)$ in the Arthur-Campoli relations. These relations may then be reformulated as linear relations on differentiated families of representations.

In Section 4 we simplify the definition of $\mathrm{PW}^{D}(G, K)$. First of all, due to the intertwining relations in the definition, the space can be defined in terms of just the minimal parabolic subgroup $P_{0}$. Next, the intertwining conditions (b) turn out to be a consequence of the invariant subspace conditions (a).

In the final Section 5, we bring into play the Hecke algebra $\mathbb{H}(G, K)$ consisting of all bi-K-finite distributions on $G$ supported by $K$. The importance of this algebra for representation theory is based on the fact that the category of Harish-Chandra modules is isomorphic to the category of finitely generated admissible modules for this algebra.

A key lemma in this section is the following. For $(\pi, V)$ a Harish-Chandra module, let $\operatorname{End}(\pi)^{\#}$ denote the space of $K \times K$-finite endomorphisms $\varphi$ of $V$ with the property that for every positive integer $n$ the Cartesian power $\varphi^{\times n}$ preserves all invariant subspaces of $V^{\times n}$. The mentioned key lemma asserts that

$$
\operatorname{End}(\pi)^{\#}=\operatorname{image}(\mathbb{H}(G, K)) \subset \operatorname{End}(V) .
$$

It follows from this lemma that the Paley-Wiener space $\mathrm{PW}^{D}(G, K)$ allows the following description in terms of the Hecke algebra. For every finite dimensional $\mathcal{O}_{0}$-module $E$, and all finite sets $\Xi \subset M^{\wedge}$ and $\Lambda \subset \mathfrak{a}_{\mathbb{C}}^{*}$, we define the representation $\pi_{E, \Xi, \Lambda}$ to be the direct sum of the representations $\pi_{P_{0}, \xi, \lambda}^{(E)}$, for $(\xi, \lambda) \in \Xi \times \Lambda$. Moreover, for $\varphi \in \mathrm{PW}_{P_{0}}^{\mathrm{pre}}(G, K)$, we define the endomorphism $\varphi_{E, \Xi, \Lambda}$ of $\pi_{E, \Xi, \Lambda}$ by taking a similar direct sum. Then $\operatorname{PW}^{D}(G, K)$ maps isomorphically onto the space of $\varphi \in \mathrm{PW}_{P_{0}}^{\text {pre }}(G, K)$ such that for all $E, \Xi, \Lambda$ as above,

$$
\varphi_{E, \Xi, \Lambda} \in \pi_{E, \Xi, \Lambda}(\mathbb{H}(G, K)) .
$$

On the other hand, it follows from its definition that Arthur's PaleyWiener space $\mathrm{PW}^{A}(G, K)$ is equal to the space of $\varphi \in \mathrm{PW}_{P_{0}}^{\mathrm{pre}}(G, K)$ such that for all $E, \Xi, \Lambda$ as above, $\varphi_{E, \Xi, \lambda}$ is annihilated by the annihilator of $\pi_{E, \Xi, \Lambda}(\mathbb{H}(G, K))$ in the contragredient module. Since this condition is equivalent to $(\underline{0.6})$, it thus follows that the map (0.5) is a linear isomorphism onto $\mathrm{PW}^{A}(G, K)$ (Theorem 5.11).

Returning to the original formulation of Arthur's Paley-Wiener theorem, we finally wish to mention that the condition (0.4) may be replaced by the condition that for all $\xi_{i}, u_{i}, \lambda_{i}$ as in (0.4), there exists a $h \in \mathbb{H}(G, K)$ such that for all $i$,

$$
\varphi\left(\xi_{i}, \lambda_{i} ; u_{i}\right)=\pi_{P_{0}, \xi_{i}, \lambda_{i} ; u_{i}}(h) .
$$

This characterization is given in Subsection 5.5 where it is used to derive, from Arthur's theorem, the Paley-Wiener theorem for bi-K-invariant functions, due to S. Helgason [7] and R. Gangolli [6].

Acknowledgments: We thank Pierre Baumann for a helpful discussion, which led to a simpler proof of Lemma 5.3. The second named author was 
partially supported by a grant of The Netherlands Organization for Scientific Research, NWO, under project number 613.000.213.

\section{Notation AND PRELIMINARIES}

Throughout this paper, $G$ will be a real reductive Lie group in the HarishChandra class and $K$ a maximal compact subgroup. Let $U(\mathfrak{g})$ be the universal enveloping algebra of the complexification $\mathfrak{g}_{\mathbb{C}}$ of $\mathfrak{g}$. We denote by $X \mapsto X^{\vee}$ the anti-automorphism of $U(\mathfrak{g})$ which on $\mathfrak{g}$ is given by $X \mapsto-X$.

In this paper, locally convex spaces will always be assumed to be Hausdorff and defined over $\mathbb{C}$.

For any continuous representation $(\tau, V)$ of $K$ (in a quasi-complete locally convex space) and any class $\gamma$ in the unitary dual $K^{\wedge}$ of $K$, the $K$-isotypic component of $(\tau, V)$ of type $\gamma$ is denoted by $V_{\gamma}$. The associated $K$-equivariant projection onto $V_{\gamma}$ is denoted by $P_{\gamma}$.

For every finite subset $\theta$ of $K^{\wedge}$, we put

$$
V_{\theta}:=\oplus_{\gamma \in \theta} V_{\gamma} \quad \text { and } \quad P_{\theta}:=\oplus_{\gamma \in \theta} P_{\gamma} .
$$

For any continuous representation $(\pi, V)$ of $G$, with $V$ a quasi-complete locally convex space, let $V^{\infty}$ and $V_{K}$ denote the vector subspaces of smooth and $K$-finite elements of $V$, respectively. The first one gives rise to a subrepresentation of $\pi$ and the second one to its underlying $(\mathfrak{g}, K)$-module $\left(\pi, V_{K}\right)$.

We say that a (continuous) $G$-representation or a $(\mathfrak{g}, K)$-module is admissible if all its $K$-isotypic components are finite dimensional. A HarishChandra module is an admissible $(\mathfrak{g}, K)$-module which is finitely generated as a $U(\mathfrak{g})$-module.

The space $C^{\infty}(G)$ of complex valued smooth functions on $G$ is equipped with the left and right regular actions of $G$; the subspace $C_{c}^{\infty}(G)$ of compactly supported functions is invariant for these actions. The actions are continuous for the usual locally convex topologies on these spaces and may be dualized by taking contragredients. Let $\mathcal{E}^{\prime}(G)$ denote the space of compactly supported distributions on $G$, i.e., the topological linear dual of $C^{\infty}(G)$.

Fix a (bi-invariant) Haar measure $d x$ on $G$. Then the linear map

$$
C_{c}^{\infty}(G) \longrightarrow \mathcal{E}^{\prime}(G), \quad f \longmapsto f d x
$$

is an injective intertwining operator for both $G$-action. Accordingly, we will use this map to view $C_{c}^{\infty}(G)$ as a submodule of $\mathcal{E}^{\prime}(G)$.

For any continuous representation $(\pi, V)$ and any $f \in C_{c}^{\infty}(G)$, let $\pi(f)$ denote the endomorphism of $V$ defined by

$$
\pi(f) v:=\int_{G} f(x) \pi(x) v d x, \quad v \in V .
$$

Then for all $v \in V^{\infty}$ and $\xi \in\left(V^{\infty}\right)^{*}$, the following equality holds

$$
\xi(\pi(f) v)=\langle f d x, \xi(\pi(\cdot) v)\rangle ;
$$

the bracket on the right-hand side of the equation indicates the natural pairing between $\mathcal{E}^{\prime}(G)$ and $C^{\infty}(G)$.

Let $\left(\pi_{i}, V_{i}\right), i=1,2$, be two $(\mathfrak{g}, K)$-modules. The space $\operatorname{Hom}\left(V_{1}, V_{2}\right)$ of (linear) homomorphisms from $V_{1}$ to $V_{2}$ is naturally endowed with a $(\mathfrak{g} \times$ 
$\mathfrak{g}, K \times K)$-module structure. Indeed, for any $T \in \operatorname{Hom}\left(V_{1}, V_{2}\right)$,

$$
\begin{aligned}
& \left(X_{1}, X_{2}\right) T=\pi_{2}\left(X_{2}\right) \circ T-T \circ \pi_{1}\left(X_{1}\right), \quad X_{1}, X_{2} \in \mathfrak{g}, \\
& \left(k_{1}, k_{2}\right) T=\pi_{2}\left(k_{2}\right) \circ T \circ \pi_{1}\left(k_{1}^{-1}\right), \quad k_{1}, k_{2} \in K .
\end{aligned}
$$

Accordingly, the subspace $\operatorname{Hom}_{(\mathfrak{g}, K)}\left(V_{1}, V_{2}\right)$ of $(\mathfrak{g}, K)$-homomorphisms consists of the elements of $\operatorname{Hom}\left(V_{1}, V_{2}\right)$ which are invariant under the diagonal action.

Lemma 1.1. Let $(\pi, V)$ be an admissible representation of $G$ and let $\theta_{1}$ and $\theta_{2}$ be finite subsets of $K^{\wedge}$. Then the linear map

$$
C_{c}^{\infty}(G) \longrightarrow \operatorname{Hom}\left(V_{\theta_{1}}, V_{\theta_{2}}\right), \quad f \longmapsto P_{\theta_{2}} \pi(f) P_{\theta_{1}}
$$

uniquely extends to a continuous linear map from $\mathcal{E}^{\prime}(G)$ to $\operatorname{Hom}\left(V_{\theta_{1}}, V_{\theta_{2}}\right)$.

Proof. Uniqueness of the extension follows by density of $C_{c}^{\infty}(G) d x$ in $\mathcal{E}^{\prime}(G)$. Let $v \in V_{\theta_{1}}$ and $\xi \in V_{\theta_{2}}^{*}$. Then by finite dimensionality of $\operatorname{Hom}\left(V_{\theta_{1}}, V_{\theta_{2}}\right)$ it suffices to show that the linear map

$$
\mathcal{L}: C_{c}^{\infty}(G) \longrightarrow \mathbb{C}, \quad f \longmapsto \xi(\pi(f) v)
$$

extends continuously to $\mathcal{E}^{\prime}(G)$. Define the function $m \in C^{\infty}(G)$ by $m(x)=$ $\xi(\pi(x) v)$. Then $\mathcal{L}(f)=\langle f d x, m\rangle$, for $f \in C_{c}^{\infty}(G)$. Thus, $u \mapsto\langle u, m\rangle$ defines a continuous linear extension of $\mathcal{L}$ to $\mathcal{E}^{\prime}(G)$.

We consider the convolution product $*$ on $C_{c}^{\infty}(G)$ given by

$$
f * g(x)=\int_{G} f(y) g\left(y^{-1} x\right) d y,
$$

for $f, g \in C_{c}^{\infty}(G)$ and $x \in G$. It defines an algebra structure on $C_{c}^{\infty}(G)$. The subspace $C_{c}^{\infty}(G, K)$ of left and right $K$-finite elements in $C_{c}^{\infty}(G)$ is closed under convolution, hence a subalgebra of $C_{c}^{\infty}(G)$.

The convolution product has a unique extension to a separately continuous bilinear map $\mathcal{E}^{\prime}(G) \times \mathcal{E}^{\prime}(G) \rightarrow \mathcal{E}^{\prime}(G)$, denoted $(u, v) \mapsto u * v$. This turns $\mathcal{E}^{\prime}(G)$ into an algebra. It is readily seen that the subspace $\mathcal{E}^{\prime}(G, K)$ of left and right $K$-finite elements in $\mathcal{E}^{\prime}(G)$ is closed under convolution, hence a subalgebra. Likewise, the subspace $\mathcal{E}_{K}^{\prime}(G)$ of distributions with support in $K$ is a subalgebra, and so is the intersection

$$
\mathbb{H}(G, K):=\mathcal{E}_{K}^{\prime}(G) \cap \mathcal{E}^{\prime}(G, K) .
$$

The latter is also called the Hecke algebra of the pair $(G, K)$ and is sometimes denoted by $\mathbb{H}$ for simplicity.

From Lemma 1.1, we obtain the continuous linear map

$$
\mathcal{E}^{\prime}(G, K) \rightarrow \operatorname{End}\left(V_{K}\right)_{K \times K}, \quad u \mapsto \pi(u)
$$

which intertwines the $(\mathfrak{g} \times \mathfrak{g}, K \times K)$-actions. Here the space on the right is equipped with the weakest topology for which the $K \times K$-equivariant projections of finite rank are continuous. By application of Fubini's theorem we see that $\pi$ is a morphism on the convolution algebra $C_{c}^{\infty}(G, K)$, which is a dense subalgebra of $\mathcal{E}^{\prime}(G, K)$.

By separate continuity of $*$ and continuity of (1.2), it now follows that the map (1.2) is a homomorphism of algebras. 
Fix normalized Haar measure $d k$ on $K$. Then each $\varphi \in C(K)$ defines a distribution $\varphi d k$ in $\mathcal{E}_{K}^{\prime}(G)$, given by

$$
\langle\varphi d k, f\rangle=\int_{K} \varphi(k) f(k) d k .
$$

For a given representation $\gamma \in K^{\wedge}$, we define the distribution $\alpha_{\gamma} \in \mathbb{H}$ by $\alpha_{\gamma}=\operatorname{dim}(\gamma) \chi_{\gamma \vee} d k$, where $\chi_{\gamma \vee}$ denotes the character of the contragredient $\gamma^{\vee}$ of $\gamma$. Moreover, for $\theta \subset K^{\wedge}$ a finite subset, we define the element $\alpha_{\theta} \in \mathbb{H}$ by

$$
\alpha_{\theta}:=\sum_{\gamma \in \theta} \alpha_{\gamma}
$$

The functions $\alpha_{\theta}$, viewed as elements of $\mathbb{H}$, will later be seen to define an approximation of the identity in $\mathbb{H}$.

Let $\theta_{1}, \theta_{2} \subset K^{\wedge}$ be finite subsets. We agree to write $\mathcal{E}^{\prime}(G, K)_{\theta_{1} \theta_{2}}$ for the space of distributions $\varphi \in \mathcal{E}^{\prime}(G, K)$ satisfying

$$
\alpha_{\theta_{1}} * \varphi * \alpha_{\theta_{2}}=\varphi \text {. }
$$

Then $\mathcal{E}^{\prime}(G, K)_{\theta_{1} \theta_{2}}$ consists of the distributions in $\mathcal{E}^{\prime}(G, K)$ of left $K$-type in $\theta_{1}$ and of right $K$-type in $\theta_{2}^{\vee}=\left\{\gamma^{\vee} \mid \gamma \in \theta_{2}\right\}$. Similarly, we write

$$
\begin{aligned}
C_{c}^{\infty}(G, K)_{\theta_{1} \theta_{2}} & :=C_{c}^{\infty}(G, K) \cap \mathcal{E}^{\prime}(G, K)_{\theta_{1} \theta_{2}}, \quad \text { and } \\
\mathbb{H}_{\theta_{1} \theta_{2}} & :=\mathbb{H} \cap \mathcal{E}^{\prime}(G, K)_{\theta_{1} \theta_{2}} .
\end{aligned}
$$

so that, for all admissible $G$-representations $(\pi, V)$ and $(\tau, U)$,

$$
\operatorname{Hom}(U, V)_{\theta_{1} \theta_{2}} \simeq \operatorname{Hom}\left(U_{\theta_{2}}, V_{\theta_{1}}\right),
$$

naturally. Viewing $\operatorname{Hom}(U, V)_{K \times K} \simeq V_{K} \otimes\left(U^{*}\right)_{K}$ as a $(\mathfrak{g} \times \mathfrak{g}, K \times K)$-module in a natural way, we see that

$$
\operatorname{Hom}(U, V)_{\theta_{1} \theta_{2}}=\operatorname{Hom}(U, V)_{\theta_{1} \otimes \theta_{2}^{\vee}} .
$$

In particular, it is readily seen that

$$
\pi\left(\mathcal{E}^{\prime}(G, K)_{\theta_{1} \theta_{2}}\right) \subset \operatorname{End}(V)_{\theta_{1} \theta_{2}} \simeq \operatorname{End}\left(V_{K}\right)_{\theta_{1} \theta_{2}} .
$$

Here we note that $\operatorname{End}\left(V_{K}\right)_{K \times K} \subset \operatorname{End}(V)$, naturally.

Proposition 1.2. For any admissible representation $(\pi, V)$ of $G$,

$$
\pi\left(C_{c}^{\infty}(G, K)\right)=\pi\left(\mathcal{E}^{\prime}(G, K)\right)=\pi(\mathbb{H}) .
$$

Proof. We denote the three given subspaces of $\operatorname{End}\left(V_{K}\right)_{K \times K}$ by $E_{\infty}, E_{\mathcal{E}^{\prime}}$ and $E_{\mathbb{H}}$ respectively. Let $\beta$ be the $K$-equivariant bilinear form on $\operatorname{End}\left(V_{K}\right)_{K \times K}$ given by

$$
\beta(A, B)=\operatorname{tr}(A \circ B) .
$$

By admissibility of $\pi$ it follows that $\beta$ defines a non-degenerate pairing, which is perfect when restricted to $\operatorname{End}\left(V_{K}\right)_{\theta \theta}$, for $\theta$ any finite subset of $K^{\wedge}$. Therefore, it suffices to show that the $\beta$-orthocomplements $E_{\infty}^{\perp}, E_{\mathcal{E}^{\prime}}^{\perp}$ and $\mathbb{H}^{\perp}$ are equal. Thus, let $T \in \operatorname{End}\left(V_{K}\right)_{K \times K}$. Then it suffices to show that the following assertions are equivalent:

(i) $\operatorname{tr} T \pi(x)=0$, for all $x \in G$;

(ii) $\operatorname{tr} T \pi(f)=0$, for all $f \in C_{c}^{\infty}(G, K)$;

(iii) $\operatorname{tr} T \pi(f)=0$, for all $f \in \mathcal{E}^{\prime}(G, K)$;

(iv) $\operatorname{tr} T \pi(f)=0$, for all $f \in \mathbb{H}$. 
Obviously, (i) implies (ii). By density of $C_{c}^{\infty}(G, K)_{\theta \theta}$ in $\mathcal{E}^{\prime}(G, K)_{\theta \theta}$, for every finite subset $\theta \subset K^{\wedge}$, if follows that (ii) implies (iii). Moreover, (iii) implies (iv). We will finish the proof by showing that (iv) implies (i).

Assume (iv). For $x \in G$, we define $M(x):=\operatorname{tr} T \pi(x)$. By admissibility and $K \times K$-finiteness of $T$ it follows that $M$ is an analytic function on $G$. From (iv) it follows that $\langle f, M\rangle=0$ for any $f \in \mathbb{H}$. Fix a finite subset $\theta \subset K^{\wedge}$ such that $M \in C_{c}^{\infty}(G, K)_{\theta \theta}$. Then $M=\alpha_{\theta} * M * \alpha_{\theta}$. Let $u \in U(\mathfrak{g})$, and set

$$
f:=\alpha_{\theta} *\left(L_{u} \delta_{e}\right) * \alpha_{\theta} \in \mathbb{H},
$$

where $\delta_{e}$ is the Dirac measure at the unit element $e$ of $G$. Then

$$
0=\langle f, M\rangle=\left\langle L_{u} \delta_{e}, M\right\rangle=\left(L_{\breve{u}} M\right)(e) .
$$

By analyticity this implies that $M$ vanishes on the identity component $G_{0}$ of $G$. By $K$-stability of $\mathbb{H}$, we deduce that $M$ vanishes on $K G_{0}$. Since $G$ is of the Harish-Chandra class, this means that $M=0$ on $G$.

Corollary 1.3. Let $(\pi, V)$ be an admissible representation of $G$ and assume that $\theta_{1}$ and $\theta_{2}$ are finite subsets of $K^{\wedge}$. Then

$$
\begin{aligned}
\pi\left(C_{c}^{\infty}(G, K)_{\theta_{1} \theta_{2}}\right) & =\pi\left(\mathcal{E}^{\prime}(G, K)_{\theta_{1} \theta_{2}}\right) \\
& =\pi\left(\mathbb{H}_{\theta_{1} \theta_{2}}\right) \\
& =\pi(\mathbb{H}) \cap \operatorname{End}\left(V_{K}\right)_{\theta_{1} \theta_{2}}
\end{aligned}
$$

Proof. This follows from Proposition 1.2 by using $K$-equivariant projections.

\section{Holomorphic FAMILIES OF REPRESENTATIONS AND THEIR DERIVATIVES}

Let $\mathfrak{v}$ be a finite dimensional real linear space. For any open subset $\Omega$ of its complexification $\mathfrak{v}_{\mathbb{C}}$, we denote by $\mathcal{O}(\Omega)$ the space of holomorphic $\mathbb{C}$ valued functions on $\Omega$, endowed with the topology of uniform convergence on compact subsets.

For $\mu \in \mathfrak{v}_{\mathbb{C}}$, we denote by $\mathcal{O}_{\mu}$ the algebra of germs at $\mu$ of holomorphic functions defined on a neighborhood of $\mu$. For any $\Omega$ as above, $\mu \in \Omega$ and $f \in \mathcal{O}(\Omega)$, the germ of $f$ at $\mu$ is denoted by $\gamma_{\mu}(f) \in \mathcal{O}_{\mu}$.

Let $\mathcal{P}=\mathcal{P}\left(\mathfrak{v}_{\mathbb{C}}\right)$ denote the algebra of polynomial functions $\mathfrak{v}_{\mathbb{C}} \rightarrow \mathbb{C}$. Then the map $p \mapsto \gamma_{0}(p)$ is an embedding of algebras, $\mathcal{P} \hookrightarrow \mathcal{O}_{0}$. Accordingly, we shall view $\mathcal{P}$ as a subalgebra of $\mathcal{O}_{0}$.

The ring $\mathcal{O}_{0}$ is local; its unique maximal ideal $\mathcal{M}$ consists of the elements vanishing at 0 . An ideal $\mathcal{I} \triangleleft \mathcal{O}_{0}$ is said to be cofinite if the quotient $\mathcal{O}_{0} / \mathcal{I}$ is finite dimensional as a vector space over $\mathbb{C}$. For $k \in \mathbb{N}$, let $\mathcal{P}_{k}$ denote the space of polynomial functions $\mathfrak{v}_{\mathbb{C}} \rightarrow \mathbb{C}$ of degree at most $k$. Then

$$
\mathcal{O}_{0}=\mathcal{P}_{k} \oplus \mathcal{M}^{k+1} \text {. }
$$

Therefore, the ideal $\mathcal{M}^{k+1}$ is cofinite in $\mathcal{O}_{0}$.

Lemma 2.1. Let $\mathcal{I}$ be an ideal in $\mathcal{O}_{0}$. Then the following assertions are equivalent:

(a) the ideal $\mathcal{I}$ is cofinite;

(b) there exists a $k \in \mathbb{N}$ such that $\mathcal{M}^{k+1} \subset \mathcal{I}$. 
Proof. As $\mathcal{M}^{k+1}$ is cofinite, (b) implies (a). Conversely, assume (a).

The space $V=\mathcal{O}_{0} / \mathcal{I}$ is a finite dimensional vector space, and an $\mathcal{O}_{0^{-}}$ module for left multiplication. The associated algebra homomorphism $\mathcal{O}_{0} \rightarrow$ $\operatorname{End}(V)$ is denoted by $\Lambda$. As $\mathcal{O}_{0}$ is a commutative algebra and $V$ is finite dimensional, there exists a positive integer $p$ such that the module $V$ decomposes as a finite direct sum of generalized weight spaces

$$
V_{\chi}:=\bigcap_{f \in \mathcal{O}_{0}} \operatorname{ker}\left(\Lambda(f)-\chi(f) \operatorname{id}_{V}\right)^{p}
$$

with $\chi \in \widehat{\mathcal{O}}_{0}:=\operatorname{Hom}\left(\mathcal{O}_{0}, \mathbb{C}\right)$. Since $\mathcal{O}_{0}$ is a local ring with maximal ideal $\mathcal{M}$, the set $\widehat{\mathcal{O}}_{0}$ of characters consists of the single element $\chi_{0}: g \mapsto g(0)$. It follows that $V=V_{\chi_{0}}$, so that $(f-f(0))^{p} \in \mathcal{I}$ for all $f \in \mathcal{O}_{0}$. In particular, $f^{p} \in \mathcal{I}$ for all $f \in \mathcal{M}$. As an ideal, $\mathcal{M}$ is generated by $n$ elements. Hence, $\mathcal{M}^{k+1} \subset \mathcal{I}$, for $k \geq n p-1$.

2.1. The derivation process. For each vector $X \in \mathfrak{v}$ we denote by $\partial_{X}$ the first order differential operator given by $\partial_{X} \varphi(a)=\left.\frac{d}{d z} \varphi(a+z X)\right|_{z=0}$ for $a \in \mathfrak{v}_{\mathbb{C}}$ and $\varphi$ a holomorphic function defined on a neighborhood of $a$ in $\mathfrak{v}_{\mathbb{C}}$. The map $X \mapsto \partial_{X}$ has a unique extension to an algebra isomorphism $u \mapsto \partial_{u}$ from the symmetric algebra $S(\mathfrak{v})$ of $\mathfrak{v}_{\mathbb{C}}$ onto the algebra of constant coefficient (holomorphic) differential operators on $\mathfrak{v}_{\mathbb{C}}$. We will follow HarishChandra's convention to write

$$
\varphi(a ; u):=\partial_{u} \varphi(a)
$$

for $\varphi$ a holomorphic function defined on a neighborhood of $a$.

We define the pairing $\langle\cdot, \cdot\rangle$ between $\mathcal{O}_{0}$ and $S(\mathfrak{v})$ by

$$
\begin{aligned}
\mathcal{O}_{0} \times S(\mathfrak{v}) & \rightarrow \mathbb{C} \\
(\varphi, u) & \mapsto \varphi(0 ; u) .
\end{aligned}
$$

For a given cofinite ideal $\mathcal{I} \triangleleft \mathcal{O}_{0}$, let $S_{\mathcal{I}}(\mathfrak{v})$ denote the annihilator of $\mathcal{I}$ in $S(\mathfrak{v})$ relative to this pairing. For $k \in \mathbb{N}$, let $S_{k}(\mathfrak{v})$ be the linear subspace of $S(\mathfrak{v})$ consisting of the elements of order at most $k$.

Lemma 2.2. Let $k \in \mathbb{N}$. Then

(a) $S_{k}(\mathfrak{v})=S_{\mathcal{M}^{k+1}}(\mathfrak{v})$;

(b) the pairing (2.3) induces a perfect pairing $\left(\mathcal{O}_{0} / \mathcal{M}^{k+1}\right) \times S_{k}(\mathfrak{v}) \rightarrow \mathbb{C}$.

Proof. The pairing $\langle\cdot, \cdot\rangle$, defined in (2.3), vanishes on $\mathcal{M}^{k+1} \times S_{k}(\mathfrak{v})$. Thus, $S_{k}(\mathfrak{v}) \subset S_{\mathcal{M}^{k+1}}(\mathfrak{v})$.

From the decomposition $\mathcal{O}_{0}=\mathcal{M}^{k+1} \oplus \mathcal{P}_{k}$, and non-degeneracy of the pairing, it follows that $S_{\mathcal{M}^{k+1}}(\mathfrak{v}) \hookrightarrow \mathcal{P}_{k}^{*}$. In particular, the dimension of $S_{\mathcal{M}^{k+1}}(\mathfrak{v})$ does not exceed the dimension of $\mathcal{P}_{k}$, which in turn equals the dimension of $S_{k}(\mathfrak{v})$. Assertion (a) now follows.

It also follows that the induced embedding $S_{k}(\mathfrak{v})=S_{\mathcal{M}^{k+1}}(\mathfrak{v}) \hookrightarrow \mathcal{P}_{k}^{*} \simeq$ $\left(\mathcal{O}_{0} / \mathcal{M}^{k+1}\right)^{*}$ is an isomorphism onto. By finite dimensionality, this implies assertion (b).

Lemma 2.3. Let $\mathcal{I}$ be a cofinite ideal of $\mathcal{O}_{0}$. Then the pairing (2.3) induces a linear isomorphism $S_{\mathcal{I}}(\mathfrak{v}) \simeq\left(\mathcal{O}_{0} / \mathcal{I}\right)^{*}$. 
Proof. By Lemma 2.1 there exists a $k \in \mathbb{N}$ such that $\mathcal{M}^{k+1} \subset \mathcal{I}$. This inclusion induces an embedding of $\left(\mathcal{O}_{0} / \mathcal{I}\right)^{*}$ into $\left(\mathcal{O}_{0} / \mathcal{M}^{k+1}\right)^{*}$. In view of Lemma 2.2, it follows that the pairing induces an embedding $\left(\mathcal{O}_{0} / \mathcal{I}\right)^{*} \hookrightarrow$ $S(\mathfrak{v})$. Its image is contained in the annihilator $S_{\mathcal{I}}(\mathfrak{v})$, by definition of the latter. On the other hand, the pairing induces an inclusion $S_{\mathcal{I}}(\mathfrak{v}) \hookrightarrow \mathcal{O}_{0}^{*}$ and elements of $S_{\mathcal{I}}(\mathfrak{v})$ vanish on $\mathcal{I}$, so that $S_{\mathcal{I}}(\mathfrak{v}) \hookrightarrow\left(\mathcal{O}_{0} / \mathcal{I}\right)^{*}$. The result follows.

For $\mu \in \mathfrak{v}_{\mathbb{C}}$ we denote by $T_{\mu}$ the translation in $\mathfrak{v}_{\mathbb{C}}$ given by $\nu \mapsto \nu+\mu$. We note that the pull-back map $T_{\mu}^{*}: \varphi \mapsto \varphi \circ T_{\mu}$ induces a ring isomorphism from $\mathcal{O}_{\mu}$ onto $\mathcal{O}_{0}$.

Let $\mathcal{I} \triangleleft \mathcal{O}_{0}$ be a cofinite ideal and let $\Omega$ be an open subset of $\mathfrak{v}_{\mathbb{C}}$. Then for every $f \in \mathcal{O}(\Omega)$ and each $\mu \in \Omega$ we define

$$
J_{\mathcal{I}} f(\mu):=\operatorname{pr}_{\mathcal{I}}\left(\gamma_{0}\left(T_{\mu}^{*} f\right)\right) \in \mathcal{O}_{0} / \mathcal{I}
$$

where $\operatorname{pr}_{\mathcal{I}}$ denotes the projection of $\mathcal{O}_{0}$ onto $\mathcal{O}_{0} / \mathcal{I}$.

In the following lemma, which is a straightforward consequence of the definitions, $\langle\cdot, \cdot\rangle$ denotes the pairing induced by (2.3), see Lemma 2.3.

Lemma 2.4. Let $f \in \mathcal{O}(\Omega)$. Then for all $\mu \in \Omega$,

$$
\left\langle J_{\mathcal{I}} f(\mu), u\right\rangle=f(\mu ; u), \quad u \in S_{\mathcal{I}}(\mathfrak{v}) .
$$

Corollary 2.5. The map $f \mapsto J_{\mathcal{I}} f$ defines a continuous algebra homomorphism from $\mathcal{O}(\Omega)$ to $\mathcal{O}\left(\Omega, \mathcal{O}_{0} / \mathcal{I}\right)$.

Proof. Let $u \in S(\mathfrak{v})$. For every $f \in \mathcal{O}(\Omega)$, the function $\partial_{u} f=f(\cdot ; u)$ belongs to $\mathcal{O}(\Omega)$. Moreover, the map $\partial_{u}$ is a continuous linear endomorphism of $\mathcal{O}(\Omega)$. In view of Lemma 2.4, it follows that for each $\xi \in\left(\mathcal{O}_{0} / I\right)^{*}$ the map $f \mapsto \xi \circ\left[J_{\mathcal{I}}(f)\right]$ is a continuous linear endomorphism of $\mathcal{O}(\Omega)$. By finite dimensionality of $\mathcal{O}_{0} / \mathcal{I}$, it follows that $J_{\mathcal{I}}$ is continuous.

The assertion that $J_{\mathcal{I}}$ is an algebra homomorphism follows from combining the observations that $T_{\mu}^{*}, \gamma_{0}$, and $\operatorname{pr}_{\mathcal{I}}$ are algebra homomorphisms.

Example 2.6. Let $\xi \in \mathfrak{v}_{\mathbb{C}}^{*}$. Denote by $e^{\xi}$ the holomorphic function on $\mathfrak{v}_{\mathbb{C}}$ given by:

$$
e^{\xi}(\mu):=e^{\xi(\mu)}, \quad \mu \in \mathfrak{v}_{\mathbb{C}} .
$$

In terms of the canonical identification of the symmetric algebra $S(\mathfrak{v})$ with the algebra $\mathcal{P}\left(\mathfrak{v}_{\mathbb{C}}^{*}\right)$ of polynomial functions on $\mathfrak{v}_{\mathbb{C}}^{*}$ we have $\partial_{u} e^{\xi}=u(\xi) e^{\xi}$, for $u \in S(\mathfrak{v})$. Hence, if $\mathcal{I}$ is a cofinite ideal in $\mathcal{O}_{0}$, then for all $\mu \in \mathfrak{v}_{\mathbb{C}}$ and $u \in S_{\mathcal{I}}(\mathfrak{v})$,

(i) $\left\langle J_{\mathcal{I}} e^{\xi}(\mu), u\right\rangle=u(\xi) e^{\xi(\mu)}$,

(ii) $J_{\mathcal{I}} e^{\xi}(\mu)=e^{\xi(\mu)} \operatorname{pr}_{\mathcal{I}} \circ \gamma_{0}\left(e^{\xi}\right)$.

Definition 2.7. Let $E$ be a finite dimensional $\mathcal{O}_{0}$-module. For every $f \in$ $\mathcal{O}(\Omega)$ and all $\mu \in \Omega$, we define $f^{(E)}(\mu) \in \operatorname{End}(E)$ by:

$$
f^{(E)}(\mu) e:=\gamma_{0}\left(T_{\mu}^{*} f\right) \cdot e, \quad e \in E .
$$

Example 2.8. If $E=\mathcal{O}_{0} / \mathcal{I}$ for some cofinite ideal $\mathcal{I}$ of $\mathcal{O}_{0}$, then, for any $f \in \mathcal{O}(\Omega)$,

$$
J_{\mathcal{I}} f(\mu)=f^{(E)}(\mu)(1+\mathcal{I}), \quad \mu \in \Omega
$$


Let $A$ be an algebra and $E$ an $A$-module. We denote by $\operatorname{ann}_{A}(E)$ the annihilator of $E$ in $A$, i.e., the kernel of the natural algebra homomorphism $A \rightarrow \operatorname{End}(E)$. If $E$ is finite dimensional, $\operatorname{ann}_{A}(E)$ is a cofinite ideal of $A$.

Lemma 2.9. Let $\Omega \subset \mathfrak{v}_{\mathbb{C}}$ be open, and let $E$ be a finite dimensional $\mathcal{O}_{0}$ module. Then for every $f \in \mathcal{O}(\Omega)$ and all $\mu \in \Omega$,

(a) $f^{(E)}(\mu) e=J_{\operatorname{ann}_{\mathcal{O}_{0}}(E)} f(\mu) \cdot e, \quad$ for all $e \in E$;

(b) $\left(T_{\mu}^{*} f\right)^{(E)}=T_{\mu}^{*} f^{(E)}$.

Proof. These formulas follow by straightforward computation.

Lemma 2.10. Let $E$ be a finite dimensional $\mathcal{O}_{0}$-module and let $\eta \in \operatorname{End}(E)^{*}$. Then there exists an element $u=u_{\eta} \in S(\mathfrak{v})$ such that

$$
\eta \circ f^{(E)}=\partial_{u} f
$$

for every open $\Omega \subset \mathfrak{v}_{\mathbb{C}}$ and all $f \in \mathcal{O}(\Omega)$.

Proof. It suffices to prove this for $\eta=e^{*} \otimes e$, with $e^{*} \in E^{*}$ and $e \in E$. Then, for $f \in \mathcal{O}(\Omega)$ and $\lambda \in \Omega$,

$$
\eta \circ f^{(E)}(\lambda)=e^{*}\left(\gamma_{0}\left(T_{\lambda}^{*} f\right) \cdot e\right) .
$$

Let $\mathcal{I}$ be the (cofinite) annihilator of $e$ in $\mathcal{O}_{0}$. Then the linear functional $L: \varphi \mapsto e^{*}(\varphi \cdot e)$ on $\mathcal{O}_{0}$ factors through a linear map $\mathcal{O}_{0} / \mathcal{I} \rightarrow \mathbb{C}$. Hence, in view of Lemma 2.3, there exists an element $u \in S_{\mathcal{I}}(\mathfrak{v})$ such that $L(\varphi)=$ $\partial_{u} \varphi(0)$ for all $\varphi \in \mathcal{O}_{0}$. It follows that the expression on the right-hand side of (2.5) equals $\partial_{u} f(\lambda)$.

We shall also need a kind of converse to the above lemma.

Lemma 2.11. Let $F \subset S(\mathfrak{v})$ be a finite subset. Then there exists a finite dimensional $\mathcal{O}_{0}$-module $E$, and linear functionals $\eta_{u} \in \operatorname{End}(E)^{*}$, for $u \in F$, such that

$$
\eta_{u} \circ f^{(E)}=\partial_{u} f
$$

for every $u \in F$, every open $\Omega \subset \mathfrak{v}_{\mathbb{C}}$ and all $f \in \mathcal{O}(\Omega)$.

Proof. By taking direct sums of finite dimensional $\mathcal{O}_{0}$-modules we may reduce to the case that $F$ consists of a single element $u \in S(\mathfrak{v})$. Let $k$ be the order of $u$. Then $\varphi \mapsto \varphi(0 ; u)$ defines a linear functional $e^{*}$ on $\mathcal{O}_{0} / \mathcal{I}$, for $\mathcal{I}=\mathcal{M}^{k+1}$. We put $E=\mathcal{O}_{0} / \mathcal{I}$ and let $e$ denote the image of $1 \in \mathcal{O}_{0}$ in $E$. Let $\eta=e^{*} \otimes e$ be the linear functional on $\operatorname{End}(E)$ defined by $T \mapsto e^{*}(T e)$. Then for all $f \in \mathcal{O}(\Omega)$ and all $\lambda \in \Omega$ we have

$$
\eta \circ f^{(E)}(\lambda)=\eta\left(\gamma_{0}\left(T_{\lambda}^{*} f\right)\right)=e^{*}\left(\gamma_{0}\left(T_{\lambda}^{*} f\right) \cdot e\right)=\partial_{u}\left(\gamma_{0}\left(T_{\lambda}^{*} f\right)\right)(0)=\partial_{u} f(\lambda) .
$$

We retain the assumption that $\Omega$ is an open subset of $\mathfrak{v}_{\mathbb{C}}$.

Corollary 2.12. Let $E$ be a finite dimensional $\mathcal{O}_{0}$-module. Then $f \mapsto f^{(E)}$ is a continuous algebra homomorphism from $\mathcal{O}(\Omega)$ to $\mathcal{O}(\Omega, \operatorname{End}(E)$ ).

Proof. The map is an algebra homomorphism by Lemma 2.9 (a) and Corollary 2.5. The continuity is an immediate consequence of Lemma 2.10. 
We agree to use the following notation for the map of Corollary 2.12

$$
J^{(E)}: f \mapsto f^{(E)}, \quad \mathcal{O}(\Omega) \rightarrow \mathcal{O}(\Omega, \operatorname{End}(E)) .
$$

The following property is an immediate consequence of the definitions; here we keep in mind that $\operatorname{End}(E) \oplus \operatorname{End}(F) \hookrightarrow \operatorname{End}(E \oplus F)$, naturally.

Property 2.13. Let $E, F$ be two finite dimensional $\mathcal{O}_{0}$-modules. Then for every $f \in \mathcal{O}(\Omega)$,

$$
f^{(E \oplus F)}=f^{(E)} \oplus f^{(F)} .
$$

To prepare for deriving more properties of the map $J^{(E)}$, we formulate a few results on finite dimensional $\mathcal{O}_{0}$-modules.

Lemma 2.14. Let $E$ be a finite dimensional $\mathcal{O}_{0}$-module. Then $E$ is cyclic if and only if there exists a cofinite ideal $\mathcal{I}$ of $\mathcal{O}_{0}$ such that $E \simeq \mathcal{O}_{0} / \mathcal{I}$.

Proof. Straightforward.

Corollary 2.15. Let $E$ be a finite dimensional $\mathcal{O}_{0}$-module. Then there exist finitely many cofinite ideals $\mathcal{I}_{1}, \ldots, \mathcal{I}_{n}$ of $\mathcal{O}_{0}$ such that $E$ is a quotient of the direct sum $\mathcal{O}_{0} / \mathcal{I}_{1} \oplus \cdots \oplus \mathcal{O}_{0} / \mathcal{I}_{n}$ of $\mathcal{O}_{0}$-modules.

In particular, there exist $k, N \in \mathbb{N}$ such that $E$ is a quotient of the $\mathcal{O}_{0}$ module $\left(\mathcal{O}_{0} / \mathcal{M}^{k+1}\right)^{N}$ for some $k, N \in \mathbb{N}$.

Proof. The first assertion results from the previous lemma. The second follows from Lemma 2.1 .

Besides the decomposition (2.1), we have the following decomposition of $\mathcal{P}=\mathcal{P}\left(\mathfrak{v}_{\mathbb{C}}\right)$, for $k \in \mathbb{N}$,

$$
\mathcal{P}=\mathcal{P}_{k} \oplus(\mathcal{M} \cap \mathcal{P})^{k+1} .
$$

Hence, the embedding $\mathcal{P} \hookrightarrow \mathcal{O}_{0}$ induces, for each $k \in \mathbb{N}$, an isomorphism of algebras

$$
\iota_{k}: \mathcal{P} /(\mathcal{M} \cap \mathcal{P})^{k+1} \stackrel{\sim}{\longrightarrow} \mathcal{O}_{0} / \mathcal{M}^{k+1} .
$$

It follows that $\mathcal{P} /(\mathcal{M} \cap \mathcal{P})^{k+1}$ is a local ring, with unique maximal ideal equal to $(\mathcal{M} \cap \mathcal{P}) /(\mathcal{M} \cap \mathcal{P})^{k+1}$. Thus, if $\widetilde{\mathcal{I}} \subset \mathcal{P}$ is an ideal with $(\mathcal{M} \cap \mathcal{P})^{k+1} \subset \widetilde{\mathcal{I}}$, then $\widetilde{\mathcal{I}} \subset \mathcal{M}$.

Lemma 2.16. Let $\widetilde{\mathcal{I}}$ be an ideal of $\mathcal{P}$. Then the following assertions are equivalent:

(a) there exists a $k \in \mathbb{N}$ such that $(\mathcal{M} \cap \mathcal{P})^{k+1} \subset \widetilde{\mathcal{I}}$;

(b) there exists an ideal $\mathcal{I} \triangleleft \mathcal{O}_{0}$ of finite codimension, such that $\widetilde{\mathcal{I}}=$ $\mathcal{I} \cap \mathcal{P}$.

If any of these conditions is fulfilled, then the ideal $\mathcal{I}$ in (b) is unique and the embedding of $\mathcal{P}$ into $\mathcal{O}_{0}$ induces an isomorphism of algebras $\mathcal{P} / \widetilde{\mathcal{I}} \rightarrow \mathcal{O}_{0} / \mathcal{I}$.

Proof. Assume (a). The image $\tilde{\mathcal{I}}^{\prime}$ of $\widetilde{\mathcal{I}}$ in $\mathcal{P} /(\mathcal{M} \cap \mathcal{P})^{k+1}$ is an ideal. Its image $\iota_{k}\left(\tilde{\mathcal{I}}^{\prime}\right)$ is an ideal of $\mathcal{O}_{0} / \mathcal{M}^{k+1}$. Let $\mathcal{I}$ be the preimage of $\iota_{k}\left(\tilde{\mathcal{I}}^{\prime}\right)$ in $\mathcal{O}_{0}$. Then the following diagram commutes

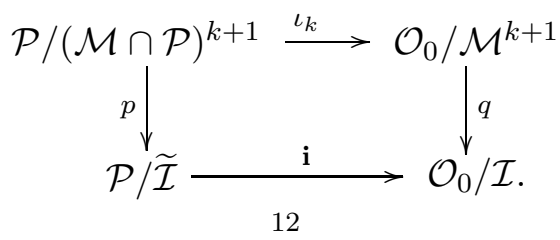


Here $\mathbf{i}$ is induced by the inclusion map $\mathcal{P} \hookrightarrow \mathcal{O}_{0}$. The kernel of $p$ equals $\widetilde{\mathcal{I}}^{\prime}$ and the kernel of $q$ equals $\iota_{k}\left(\widetilde{\mathcal{I}}^{\prime}\right)$. As $\iota_{k}$ is an isomorphism of algebras, it follows that $\mathbf{i}$ is an isomorphism of algebras, and (b) is immediate.

Conversely, assume (b). Let $\mathcal{I}_{j} \triangleleft \mathcal{O}_{0}$ be ideals such that $\widetilde{\mathcal{I}}=\mathcal{I}_{j} \cap \mathcal{P}$, for $j=1,2$. We will complete the proof by showing that (a) holds and that $\mathcal{I}_{1}=\mathcal{I}_{2}$.

Since $\mathcal{I}_{1}$ and $\mathcal{I}_{2}$ are cofinite, there exists a constant $k \in \mathbb{N}$ such that $\mathcal{M}^{k+1} \subset \mathcal{I}_{j}$, for both $j=1,2$. Therefore,

$$
(\mathcal{M} \cap \mathcal{P})^{k+1} \subset\left(\mathcal{M}^{k+1} \cap \mathcal{P}\right) \subset \widetilde{\mathcal{I}}
$$

and (a) follows. Moreover, for each $j=1,2$ we have the following commutative diagram:

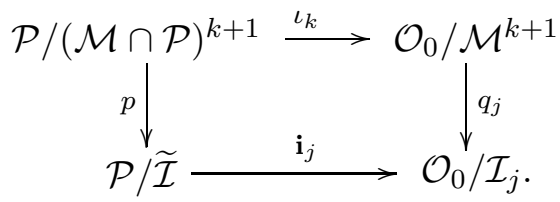

From the assumption on $\mathcal{I}_{j}$ it follows that the map $\mathbf{i}_{j}$ is injective. Moreover, since $\iota_{k}$ and $q_{j}$ are surjective, it follows that $\mathbf{i}_{j}$ is an isomorphism of algebras, for $j=1,2$. This implies that $\iota_{k}(\operatorname{ker} p)=\operatorname{ker}\left(q_{j}\right)$. Since $\mathcal{I}_{j}$ equals the preimage of $\operatorname{ker}\left(q_{j}\right)$ in $\mathcal{O}_{0}$, for $j=1,2$, it follows that $\mathcal{I}_{1}=\mathcal{I}_{2}$.

Let $\mathrm{FM}_{\mathcal{O}_{0}}$ denote the category of finite dimensional $\mathcal{O}_{0}$-modules and $\mathrm{FM}_{\mathcal{P}}$ the category of finite dimensional $\mathcal{P}$-modules $E$ for which there exists a $k \in \mathbb{N}$ such that

$$
(\mathcal{M} \cap \mathcal{P})^{k+1} \subset \operatorname{ann}_{\mathcal{P}}(E) .
$$

If $E, F$ belong to $\mathrm{FM}_{\mathcal{P}}$ then the $\mathrm{FM}_{\mathcal{P}}$-morphisms are defined to be the $\mathcal{P}$ module homomorphisms $E \rightarrow F$. We observe that their kernels and images belong to the category $\mathrm{FM}_{\mathcal{P}}$ as well.

If $E$ is a finite dimensional $\mathcal{O}_{0}$-module, then its annihilator $\mathcal{I}$ is cofinite. Since $\mathcal{P} \hookrightarrow \mathcal{O}_{0}$, the space $E$ is a finite dimensional $\mathcal{P}$-module as well and its annihilator $\widetilde{\mathcal{I}}:=\operatorname{ann}_{\mathcal{P}}(E)$ in $\mathcal{P}$ is given by $\widetilde{\mathcal{I}}=\mathcal{I} \cap \mathcal{P}$. Furthermore, by Lemma 2.1 there exists a $k \in \mathbb{N}$ such that

$$
(\mathcal{M} \cap \mathcal{P})^{k+1} \subset \operatorname{ann}_{\mathcal{P}}(E) .
$$

We conclude that there is a well-defined forgetful functor $\mathcal{F}: \mathrm{FM}_{\mathcal{O}_{0}} \rightarrow \mathrm{FM}_{\mathcal{P}}$.

Lemma 2.17. The forgetful functor $\mathcal{F}: \mathrm{FM}_{\mathcal{O}_{0}} \rightarrow \mathrm{FM}_{\mathcal{P}}$ is an isomorphism of categories.

Proof. Let $E$ be a non-trivial $\mathcal{P}$-module from the category $\mathrm{FM}_{\mathcal{P}}$. We will first show that $E$ carries a unique compatible structure of $\mathcal{O}_{0}$-module.

The ideal $\widetilde{\mathcal{I}}=\operatorname{ann}_{\mathcal{P}}(E)$ satisfies condition (a) of Lemma 2.16, hence equals $\mathcal{P} \cap \mathcal{I}$ for a unique cofinite ideal $\mathcal{I} \triangleleft \mathcal{O}_{0}$. Via the isomorphism $\mathcal{P} / \widetilde{\mathcal{I}} \simeq \mathcal{O}_{0} / \mathcal{I}$ we equip $E$ with the structure of $\mathcal{O}_{0}$-module; this establishes existence.

For uniqueness, assume that $E$ is equipped with a compatible structure of $\mathcal{O}_{0}$-module. Let $\mathcal{I}^{\prime}$ be the annihilator of $E$ in $\mathcal{O}_{0}$. Then $\mathcal{I}^{\prime}$ is cofinite and, by compatibility, $\widetilde{\mathcal{I}}=\mathcal{I}^{\prime} \cap \mathcal{P}$. By the first part of the proof, we see that 
$\mathcal{I}^{\prime}=\mathcal{I}$. Furthermore, by compatibility it follows that the diagram

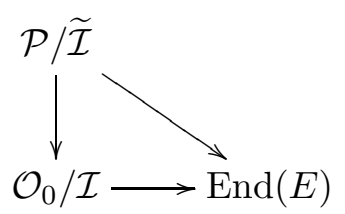

commutes. The vertical arrow is induced by the inclusion $\mathcal{P} \rightarrow \mathcal{O}_{0}$ and represents an isomorphism. This establishes uniqueness.

It follows from these considerations that each submodule of $E$ in the category $\mathrm{FM}_{\mathcal{P}}$ is a submodule for the associated compatible structure of $\mathcal{O}_{0}$-module as well.

For $E$ a finite dimensional $\mathcal{P}$-module from $\mathrm{FM}_{\mathcal{P}}$, let $\mathcal{G}(E)$ denote the same space with the uniquely defined compatible structure of $\mathcal{O}_{0}$-module. If $E_{0}$ is a submodule in the category $\mathrm{FM}_{\mathcal{P}}$, then by what we said before, $\mathcal{G}\left(E_{0}\right)$ is an $\mathcal{O}_{0}$-submodule of $\mathcal{G}(E)$. The quotient $\mathcal{G}(E) / \mathcal{G}\left(E_{0}\right)$ is an $\mathcal{O}_{0^{-}}$ module in a natural way, whose $\mathcal{O}_{0}$-module structure is compatible with the $\mathcal{P}$-module structure of $E / E_{0}$. Hence, $\mathcal{G}(E) / \mathcal{G}\left(E_{0}\right)=\mathcal{G}\left(E / E_{0}\right)$. It now follows that every morphism $f: E \rightarrow F$ in the category $\mathrm{FM}_{\mathcal{P}}$ is an $\mathcal{O}_{0^{-}}$ module homomorphism $\mathcal{G}(E) \rightarrow \mathcal{G}(F)$. We thus see that $\mathcal{G}$ defines a functor $\mathrm{FM}_{\mathcal{P}} \rightarrow \mathrm{FM}_{\mathcal{O}_{0}}$, which obviously is a two-sided inverse to $\mathcal{F}$.

Corollary 2.18. For every pair $E, F$ of finite dimensional $\mathcal{O}_{0}$-modules,

$$
\operatorname{Hom}_{\mathcal{O}_{0}}(E, F)=\operatorname{Hom}_{\mathcal{P}}(E, F) .
$$

Our next objective is to consider tensor products in the categories $\mathrm{FM}_{\mathcal{P}}$ and $\mathrm{FM}_{\mathcal{O}_{0}}$.

Let $n \in \mathbb{N}^{*}$ and consider the $n$-fold Cartesian product $\mathfrak{v}_{\mathbb{C}}^{n}$ of $\mathfrak{v}_{\mathbb{C}}$. Projection onto the $j$-th coordinate is denoted by $\mathrm{pr}_{j}$. Pull-back by $\mathrm{pr}_{j}$ defines an embedding of algebras $\operatorname{pr}_{j}^{*}: p \mapsto p \circ \operatorname{pr}_{j}, \mathcal{P} \rightarrow \mathcal{P}\left(\mathfrak{v}_{\mathbb{C}}^{n}\right)$. The multi-linear map

$$
\left(p_{1}, \ldots, p_{n}\right) \mapsto \prod_{j=1}^{n} \operatorname{pr}_{j}^{*}\left(p_{j}\right)
$$

induces an isomorphism of algebras $\mathcal{P}^{\otimes n} \rightarrow \mathcal{P}\left(\mathfrak{v}_{\mathbb{C}}^{n}\right)$, via which we shall identify the elements of these spaces. Accordingly,

$$
\left(p_{1} \otimes \cdots \otimes p_{n}\right)\left(\mu_{1}, \ldots, \mu_{n}\right)=p_{1}\left(\mu_{1}\right) \cdots p_{n}\left(\mu_{n}\right),
$$

for $p_{j} \in \mathcal{P}$ and $\mu_{j} \in \mathfrak{v}_{\mathbb{C}}$. The maximal ideal $\mathcal{M}_{\mathcal{P} \otimes n}$ in $\mathcal{P}\left(\mathfrak{v}_{\mathbb{C}}^{n}\right)$ consisting of the polynomials vanishing at 0 is now given by $\mathcal{M}_{\mathcal{P} \otimes n}=\sum_{i=1}^{n} \mathcal{M}_{\mathcal{P}, n, i}$, where

$$
\mathcal{M}_{\mathcal{P}, n, i}:=\mathcal{P} \otimes \cdots \otimes \mathcal{P} \otimes \overbrace{(\mathcal{M} \cap \mathcal{P})}^{i} \otimes \mathcal{P} \otimes \cdots \otimes \mathcal{P} .
$$

Lemma 2.19. Let $E_{1}, \ldots, E_{n}$ be finite dimensional $\mathcal{P}$-modules from the category $\mathrm{FM}_{\mathcal{P}}$. Then $E_{1} \otimes \cdots \otimes E_{n}$ is a $\mathcal{P}^{\otimes n}$-module from the category $\mathrm{FM}_{\mathcal{P} \otimes n}$.

Proof. There exists a $k \in \mathbb{N}$ such that $(\mathcal{M} \cap \mathcal{P})^{k+1}$ annihilates each of the modules $E_{i}$, for $1 \leq i \leq n$. It is now readily checked that

$$
\left(\mathcal{M}_{\mathcal{P} \otimes n}\right)^{n(k+1)} \subset \operatorname{ann}_{\mathcal{P} \otimes n}\left(E_{1} \otimes \cdots \otimes E_{n}\right) .
$$


We consider the map

$$
\alpha_{n}: \mathfrak{v}_{\mathbb{C}}^{n} \rightarrow \mathfrak{v}_{\mathbb{C}}, \quad\left(\mu_{1}, \ldots, \mu_{n}\right) \mapsto \mu_{1}+\cdots+\mu_{n} .
$$

Pull-back by $\alpha_{n}$ induces an algebra homomorphism

$$
\alpha_{n}^{*}: \mathcal{P} \longrightarrow \mathcal{P}^{\otimes n} .
$$

Lemma 2.20. The homomorphism $\alpha_{n}^{*}$ maps the maximal ideal $\mathcal{M} \cap \mathcal{P}$ of $\mathcal{P}$ into the maximal ideal $\mathcal{M}_{\mathcal{P} \otimes n}$ of $\mathcal{P}^{\otimes n}$.

Proof. The ideal $\mathcal{M} \cap \mathcal{P}$ is generated by the first order polynomials $\xi \in \mathfrak{v}_{\mathbb{C}}^{*}$. Now

$$
\alpha_{n}^{*}(\xi)=\sum_{i=1}^{n} 1 \otimes \cdots \otimes 1 \otimes \stackrel{i}{\xi} \otimes 1 \otimes \cdots \otimes 1,
$$

and the result follows.

Corollary 2.21. Let $n \in \mathbb{N}^{*}$. Via the homomorphism (2.9), every finite dimensional $\mathcal{P}^{\otimes n}$-module from the category $\mathrm{FM}_{\mathcal{P} \otimes n}$ becomes a $\mathcal{P}$-module from the category $\mathrm{FM}_{\mathcal{P}}$.

Proof. Let $E$ be a non-trivial module from the category $\mathrm{FM}_{\mathcal{P} \otimes n}$. Then there exists a $k \in \mathbb{N}$ such that

$$
\left(\mathcal{M}_{\mathcal{P} \otimes n}\right)^{k+1} \subset \operatorname{ann}_{\mathcal{P} \otimes n}(E) .
$$

By application of the previous lemma, we obtain

$$
\alpha_{n}^{*}\left((\mathcal{M} \cap \mathcal{P})^{k+1}\right) \subset \operatorname{ann}_{\mathcal{P} \otimes n}(E) .
$$

The $\mathcal{P}$-module structure on $E$ is defined by:

$$
\mathcal{P} \stackrel{\alpha_{n}^{*}}{\longrightarrow} \mathcal{P} \otimes n \longrightarrow \operatorname{End}(E) .
$$

From this we see that $\operatorname{ann}_{\mathcal{P}}(E)=\alpha_{n}^{*-1}\left(\operatorname{ann}_{\mathcal{P} \otimes n}(E)\right)$ and we infer that

$$
(\mathcal{M} \cap \mathcal{P})^{k+1} \subset \operatorname{ann}_{\mathcal{P}}(E) \text {. }
$$

Remark 2.22. If $E$ is a finite dimensional $\mathcal{P}$-module, we define

$$
m_{E}: \mathcal{P} \longrightarrow \operatorname{End}(E)
$$

by $m_{E}(p) e:=p \cdot e$, for $p \in \mathcal{P}$ and $e \in E$.

If $E_{1}, \cdots, E_{n}$ are finite dimensional modules from the category $\mathrm{FM}_{\mathcal{P}}$, then by combining Lemma 2.19 and Corollary 2.21 we may equip the tensor product $E_{1} \otimes \cdots \otimes E_{n}$ with the structure of a module from the same category $\mathrm{FM}_{\mathcal{P}}$. The module structure is given by the rule

$$
m_{E_{1} \otimes \cdots \otimes E_{n}}=\left(m_{E_{1}} \otimes \cdots \otimes m_{E_{n}}\right) \circ \alpha_{n}^{*} .
$$

In view of (2.10) this module structure is completely determined by the rule

$$
\begin{aligned}
& m_{E_{1} \otimes \cdots \otimes E_{n}}(\xi)= \\
& \quad=\sum_{i=1}^{n} \operatorname{id}_{E_{1}} \otimes \cdots \otimes \operatorname{id}_{E_{i-1}} \otimes m_{E_{i}}(\xi) \otimes \operatorname{id}_{E_{i+1}} \otimes \cdots \otimes \operatorname{id}_{E_{n}}
\end{aligned}
$$

for $\xi \in \mathfrak{v}_{\mathbb{C}}^{*} \subset \mathcal{P}$. 
Accordingly, if $E_{1}, \ldots, E_{n}$ are finite dimensional $\mathcal{O}_{0}$-modules, then in particular they are $\mathcal{P}$-modules from the category $\mathrm{FM}_{\mathcal{P}}$. We equip the module $E_{1} \otimes \cdots \otimes E_{n}$ from $\mathrm{FM}_{\mathcal{P}}$ with the unique compatible structure of $\mathcal{O}_{0}$-module.

Lemma 2.23. Let $E_{1}, \ldots, E_{n}$ be finite dimensional $\mathcal{O}_{0}$-modules. Then, as $\mathcal{O}_{0}$-modules:

$$
E_{1} \otimes \cdots \otimes E_{n} \simeq E_{1} \otimes\left(E_{2} \otimes \cdots \otimes E_{n}\right) .
$$

Proof. In view of Remark 2.22, it suffices to establish the identity as an identity of $\mathcal{P}$-modules. Thus, it suffices to show that

$$
m_{E_{1} \otimes \cdots \otimes E_{n}}=m_{E_{1} \otimes\left(E_{2} \otimes \cdots \otimes E_{n}\right)} .
$$

Since $\mathfrak{v}_{\mathbb{C}}^{*}$ generates $\mathcal{P}$, it suffices to check this identity on any element $\xi \in \mathfrak{v}_{\mathbb{C}}^{*}$. This is easily done by using the identity (2.12).

We return to the setting of an open subset $\Omega \subset \mathfrak{v}_{\mathbb{C}}$ and resume our study of the map $J^{(E)}: f \mapsto f^{(E)}, \mathcal{O}(\Omega) \rightarrow \mathcal{O}(\Omega$, $\operatorname{End}(E)$ ), introduced in (2.6), for $E$ a finite dimensional $\mathcal{O}_{0}$-module.

Lemma 2.24. The restriction to $\mathcal{P}$ of the map $J^{(E)}$ is given by

$$
\left.J^{(E)}\right|_{\mathcal{P}}=\left(\mathrm{id}_{\mathcal{P}} \otimes m_{E}\right) \circ \alpha_{2}^{*} .
$$

In particular, this restriction maps $\mathcal{P}$ into $\mathcal{P} \otimes \operatorname{End}(E)$.

Proof. Let $p \in \mathcal{P}$. For any $\mu \in \mathfrak{v}_{\mathbb{C}}$, we have $T_{\mu}^{*} p \in \mathcal{P}$, and for any $e \in E$,

$$
p^{(E)}(\mu) e=\left(T_{\mu}^{*} p\right) \cdot e=m_{E}\left(T_{\mu}^{*} p\right) e .
$$

Hence,

$$
\left.\left(\mathrm{ev}_{\mu} \otimes \operatorname{id}_{\operatorname{End}(E)}\right) \circ J^{(E)}\right|_{\mathcal{P}}=\left.m_{E} \circ T_{\mu}^{*}\right|_{\mathcal{P}} .
$$

Viewing $\mathrm{ev}_{\mu} \otimes \mathrm{id}_{\mathcal{P}}$ as a $\mathcal{P}$-valued function on $\mathcal{P} \otimes \mathcal{P}$, we may identify the map $T_{\mu}^{*}$ with $\left(\mathrm{ev}_{\mu} \otimes \mathrm{id}_{\mathcal{P}}\right) \circ \alpha_{2}^{*}$ on $\mathcal{P}$. Using the relation

$$
m_{E} \circ\left(\mathrm{ev}_{\mu} \otimes \mathrm{id}_{\mathcal{P}}\right)=\left(\mathrm{ev}_{\mu} \otimes \mathrm{id}_{\operatorname{End}(E)}\right) \circ\left(\mathrm{id}_{\mathcal{P}} \otimes m_{E}\right),
$$

we obtain the assertion of the lemma.

Proposition 2.25. Let $E_{1}$ and $E_{2}$ be two finite dimensional $\mathcal{O}_{0}$-modules. Then, for every $f \in \mathcal{O}(\Omega)$,

$$
\left(J^{\left(E_{1}\right)} \otimes \operatorname{id}_{\operatorname{End}\left(E_{2}\right)}\right) f^{\left(E_{2}\right)}=f^{\left(E_{1} \otimes E_{2}\right)} .
$$

Proof. By density of $\mathcal{P}$ in $\mathcal{O}(\Omega)$ and continuity of the maps $J^{\left(E_{1} \otimes E_{2}\right)}$ and $\left(J^{\left(E_{1}\right)} \otimes \operatorname{id}_{\operatorname{End}\left(E_{2}\right)}\right) \circ J^{\left(E_{2}\right)}$, see Corollary 2.12, it suffices to establish the validity of the identity on a fixed element $p \in \mathcal{P}$.

By definition of $J^{\left(E_{1}\right)}$, we have $\operatorname{ev}_{0} \circ J^{\left(E_{1}\right)} q=m_{E_{1}}(q)$ for each $q \in \mathcal{P}$. Using Lemma 2.24 we obtain

$$
\begin{aligned}
& \left(J^{\left(E_{1}\right)} \otimes \operatorname{id}_{\operatorname{End}\left(E_{2}\right)}\right) p^{\left(E_{2}\right)}(0)=\left(m_{E_{1}} \otimes \operatorname{id}_{\operatorname{End}\left(E_{2}\right)}\right) p^{\left(E_{2}\right)} \\
& =\left(m_{E_{1}} \otimes \operatorname{id}_{\operatorname{End}\left(E_{2}\right)}\right) \circ\left(\mathrm{id}_{\mathcal{P}} \otimes m_{E_{2}}\right)\left(\alpha_{2}^{*} p\right) \\
& =\left(m_{E_{1}} \otimes m_{E_{2}}\right) \circ \alpha_{2}^{*}(p) \\
& =m_{E_{1} \otimes E_{2}}(p)=p^{\left(E_{1} \otimes E_{2}\right)}(0) .
\end{aligned}
$$

The result now follows by translation invariance (see Lemma 2.9 (b)). 
Proposition 2.26. Let $E_{1}, \ldots, E_{n}$ be finite dimensional $\mathcal{O}_{0}$-modules. Then, for every $f \in \mathcal{O}(\Omega)$,

$$
\left(J^{\left(E_{1}\right)} \circ \cdots \circ J^{\left(E_{n}\right)}\right)(f)=f^{\left(E_{1} \otimes \cdots \otimes E_{n}\right)} .
$$

In the formulation of this proposition we have slightly abused notation, by using the abbreviation $J^{\left(E_{k}\right)}$ for $J^{\left(E_{k}\right)} \otimes \operatorname{id}_{\operatorname{End}\left(E_{k+1}\right)} \otimes \cdots \otimes \operatorname{id}_{\operatorname{End}\left(E_{n}\right)}$.

Proof. In view of Lemma 2.23, the result follows by repeated application of Proposition 2.25.

Let $\lambda \in \mathfrak{v}_{\mathbb{C}}$. By $\mathcal{I}_{\lambda}$ we denote the subset of $\mathcal{O}_{0}$ consisting of all elements $\varphi \in \mathcal{M}$ satisfying

$$
\partial_{\lambda} \varphi \in \mathcal{M}
$$

By Leibniz's rule, $\mathcal{I}_{\lambda}$ is an ideal of $\mathcal{O}_{0}$ containing $\mathcal{M}^{2}$.

Lemma 2.27. Assume $\lambda \neq 0$. Then $\mathcal{I}_{\lambda}$ is an ideal in $\mathcal{O}_{0}$ of codimension 2. If $\xi \in \mathfrak{v}_{\mathbb{C}}^{*}$ is such that $\xi(\lambda) \neq 0$, then $\mathcal{O}_{0}=\mathbb{C} 1 \oplus \mathbb{C} \xi \oplus \mathcal{I}_{\lambda}$.

Proof. Fix a basis $\xi_{1}, \ldots, \xi_{N}$ for $\mathfrak{v}_{\mathbb{C}}^{*}$ such that $\xi_{1}=\xi$ and such that $\xi_{j}(\lambda)=0$ for $j>1$. The monomials

$$
\xi^{\beta}:=\xi_{1}^{\beta_{1}} \cdots \xi_{N}^{\beta_{N}}, \quad \beta \in \mathbb{N}^{N},
$$

form a basis of the complex vector space $\mathcal{P}$. Consider a polynomial $p \in \mathcal{P}$ and write $p=\sum_{\beta} c_{\beta} \xi^{\beta}$, with $c_{\beta} \in \mathbb{C}$. Then by an easy calculation we find that

$$
p(0)=c_{0} \quad \text { and } \quad \partial_{\lambda} p(0)=c_{(1,0, \ldots, 0)} \xi_{1}(\lambda) .
$$

It follows that $p$ belongs to $\mathcal{I}_{\lambda}$ if and only if $c_{\beta}=0$ for $\beta=0$ and for $\beta=(1,0, \ldots, 0)$. This implies that $\mathcal{P}=\mathbb{C} 1 \oplus \mathbb{C} \xi_{1} \oplus\left(\mathcal{I}_{\lambda} \cap \mathcal{P}\right)$. By Lemma 2.16 the inclusion map $\mathcal{P} \rightarrow \mathcal{O}_{0}$ induces an isomorphism of algebras $\mathcal{P} /\left(\mathcal{I}_{\lambda} \cap \mathcal{P}\right) \simeq$ $\mathcal{O}_{0} / \mathcal{I}_{\lambda}$. All assertions follow.

Let $\widetilde{\mathcal{I}}_{1}, \ldots, \widetilde{\mathcal{I}}_{n}$ be a collection of ideals from $\mathcal{P}$ containing $(\mathcal{M} \cap \mathcal{P})^{k+1}$ for some $k \in \mathbb{N}$. Then the algebra homomorphism $\alpha_{n}^{*}: \mathcal{P} \rightarrow \mathcal{P}^{\otimes n}$ induces an algebra homomorphism

$$
\bar{\alpha}_{n}^{*}: \mathcal{P} \rightarrow\left(\mathcal{P} / \widetilde{\mathcal{I}}_{1}\right) \otimes \cdots \otimes\left(\mathcal{P} / \widetilde{\mathcal{I}}_{n}\right)
$$

by composition with the natural projection from $\mathcal{P}^{\otimes n}$ onto the quotient algebra on the right.

Lemma 2.28. Let $\lambda \in \mathfrak{v}_{\mathbb{C}} \backslash\{0\}$, and let $\tilde{\mathcal{I}} \triangleleft \mathcal{P}$ be an ideal with $(\mathcal{M} \cap \mathcal{P})^{k+1} \subset$ $\tilde{\mathcal{I}}$ for some $k \in \mathbb{N}$. Then the kernel of

$$
\bar{\alpha}_{2}^{*}: \mathcal{P} \longrightarrow \mathcal{P} / \tilde{\mathcal{I}} \otimes\left(\mathcal{P} / \mathcal{I}_{\lambda} \cap \mathcal{P}\right)
$$

is equal to $\left\{p \in \tilde{\mathcal{I}} \mid \partial_{\lambda} p \in \tilde{\mathcal{I}}\right\}$. In particular, this kernel contains $\mathcal{M}^{k+2} \cap \mathcal{P}$.

Proof. The last assertion follows from the first one by application of the Leibniz rule. We turn to the proof of the first assertion.

We fix a basis $\xi_{1} \ldots, \xi_{N}$ of $\mathfrak{v}_{\mathbb{C}}^{*}$ as in the proof of Lemma 2.27 and adopt the notation of that proof. Let $X_{1}, \ldots, X_{N}$ be the dual basis of $\mathfrak{v}_{\mathbb{C}}$. We note that $\lambda$ is proportional to $X_{1}$. Our first goal is to obtain a suitable formula for $\alpha_{2}^{*}$. 
Let $p \in \mathcal{P}$ and $\mu \in \mathfrak{v}_{\mathbb{C}}$. Using Taylor expansion at $\mu$ we find, for all $\nu \in \mathfrak{v}_{\mathbb{C}}$,

$$
p(\mu+\nu)=\sum_{\beta} \frac{p\left(\mu ; X^{\beta}\right)}{\beta !} \xi^{\beta}(\nu)
$$

(where $X^{\beta}:=X_{1}^{\beta_{1}} \cdots X_{N}^{\beta_{N}}$ and $\beta !:=\beta_{1} ! \cdots \beta_{N} !$ ). From this formula we deduce that

$$
\alpha_{2}^{*}(p)=\sum_{\beta} \frac{p\left(\cdot ; X^{\beta}\right)}{\beta !} \otimes \xi^{\beta} .
$$

In view of the characterization of $\mathcal{I}_{\lambda}$ in the proof of Lemma 2.27, we now see that

$$
\alpha_{2}^{*}(p)=p \otimes 1+p\left(\cdot ; X_{1}\right) \otimes \xi_{1} \quad \bmod \mathcal{P} \otimes\left(\mathcal{I}_{\lambda} \cap \mathcal{P}\right) .
$$

Furthermore, again by Lemma 2.27, we have $\mathcal{P}=\mathbb{C} 1 \oplus \mathbb{C} \xi_{1} \oplus\left(\mathcal{I}_{\lambda} \cap \mathcal{P}\right)$, so that $\mathcal{P}^{\otimes 2}=\left[\mathcal{P} \otimes\left(\mathbb{C} 1 \oplus \mathbb{C} \xi_{1}\right)\right] \oplus\left[\mathcal{P} \otimes\left(\mathcal{I}_{\lambda} \cap \mathcal{P}\right)\right]$. Accordingly, the natural projection map $\mathcal{P}^{\otimes 2} \rightarrow \mathcal{P} / \tilde{\mathcal{I}} \otimes \mathcal{P} /\left(\mathcal{I}_{\lambda} \cap \mathcal{P}\right)$ has kernel equal to

$$
\left[\widetilde{\mathcal{I}} \otimes\left(\mathbb{C} 1 \oplus \mathbb{C} \xi_{1}\right)\right] \oplus\left[\mathcal{P} \otimes\left(\mathcal{I}_{\lambda} \cap \mathcal{P}\right)\right] .
$$

Combining (2.15) and (2.16), we finally see that $\bar{\alpha}_{2}^{*}(p)=0$ if and only if

$$
p \otimes 1+p\left(\cdot ; X_{1}\right) \otimes \xi_{1} \in \widetilde{\mathcal{I}} \otimes\left(\mathbb{C} 1 \oplus \mathbb{C} \xi_{1}\right),
$$

which in turn is equivalent to $p \in \widetilde{\mathcal{I}}$ and $\partial_{\lambda} p \in \widetilde{\mathcal{I}}$.

Lemma 2.29. Let $\lambda_{1}, \ldots, \lambda_{n} \in \mathfrak{v}_{\mathbb{C}} \backslash\{0\}$. If $n>1$, the kernel of

$$
\bar{\alpha}_{n}^{*}: \mathcal{P} \longrightarrow\left(\mathcal{P} / \mathcal{I}_{\lambda_{1}} \cap \mathcal{P}\right) \otimes \cdots \otimes\left(\mathcal{P} / \mathcal{I}_{\lambda_{n}} \cap \mathcal{P}\right)
$$

equals the kernel of ${\overline{\alpha_{2}}}^{*}: \mathcal{P} \longrightarrow\left(\mathcal{P} / \operatorname{ker} \bar{\alpha}_{n-1}^{*}\right) \otimes\left(\mathcal{P} / \mathcal{I}_{\lambda_{n}} \cap \mathcal{P}\right)$, where $\bar{\alpha}_{n-1}^{*}$ denotes the composition on the left of the algebra homomorphism $\alpha_{n-1}^{*}$ with the projection $\mathcal{P}^{\otimes(n-1)} \rightarrow\left(\mathcal{P} / \mathcal{I}_{\lambda_{1}} \cap \mathcal{P}\right) \otimes \cdots \otimes\left(\mathcal{P} / \mathcal{I}_{\lambda_{n-1}} \cap \mathcal{P}\right)$.

Proof. The map $\bar{\alpha}_{n-1}^{*}$ induces an embedding (denoted by the same symbol)

$$
\bar{\alpha}_{n-1}^{*}: \mathcal{P} / \operatorname{ker} \bar{\alpha}_{n-1}^{*} \hookrightarrow\left(\mathcal{P} / \mathcal{I}_{\lambda_{1}} \cap \mathcal{P}\right) \otimes \cdots \otimes\left(\mathcal{P} / \mathcal{I}_{\lambda_{n-1}} \cap \mathcal{P}\right) .
$$

Moreover, the following diagram commutes

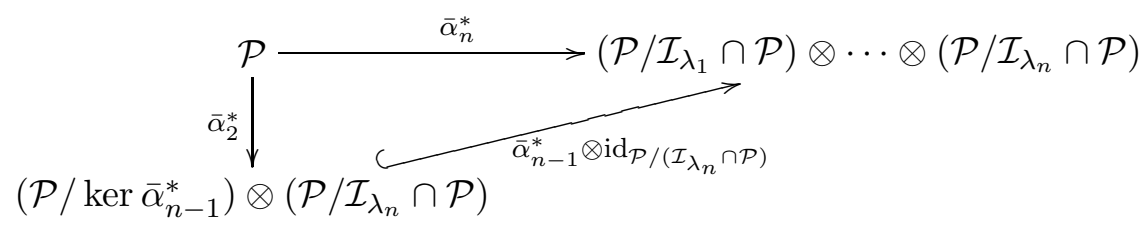

Thus, ker $\bar{\alpha}_{n}^{*}=\operatorname{ker} \bar{\alpha}_{2}^{*}$.

Lemma 2.30. Let $\lambda_{1}, \ldots, \lambda_{n} \in \mathfrak{v}_{\mathbb{C}} \backslash\{0\}$. Then the kernel of

$$
\bar{\alpha}_{n}^{*}: \mathcal{P} \longrightarrow\left(\mathcal{P} / \mathcal{I}_{\lambda_{1}} \cap \mathcal{P}\right) \otimes \cdots \otimes\left(\mathcal{P} / \mathcal{I}_{\lambda_{n}} \cap \mathcal{P}\right)
$$

consists of the elements $p$ of $\mathcal{M} \cap \mathcal{P}$ satisfying $p\left(\cdot ; \lambda_{j_{1}} \cdots \lambda_{j_{l}}\right) \in \mathcal{M}$, for all $1 \leq l \leq n, 1 \leq j_{1}<\cdots<j_{l} \leq n$. In particular, this kernel contains $\mathcal{M}^{n+1} \cap \mathcal{P}$. 
Proof. The final statement follows from the first by repeated application of the Leibniz rule. We prove the first statement by induction on $n$. If $n=1$, then $\bar{\alpha}_{1}^{*}$ is just the projection $\mathcal{P} \rightarrow \mathcal{P} / \mathcal{I}_{\lambda_{1}} \cap \mathcal{P}$, and $\operatorname{ker}\left(\bar{\alpha}_{1}^{*}\right)=\mathcal{I}_{\lambda_{1}} \cap \mathcal{P}=$ $\left\{p \in \mathcal{M} \cap \mathcal{P} \mid p\left(\cdot ; \lambda_{1}\right) \in \mathcal{M}\right\}$. Let $n \geq 2$ and assume the statement to be valid for smaller values of $n$. Using Lemmas 2.28 and 2.29, we find that

$$
\operatorname{ker}\left(\bar{\alpha}_{n}^{*}\right)=\left\{p \in \mathcal{P} \mid p \in \operatorname{ker} \bar{\alpha}_{n-1}^{*} \text { and } \partial_{\lambda_{n}} p \in \operatorname{ker} \bar{\alpha}_{n-1}^{*}\right\} .
$$

By the induction hypothesis it follows that $\operatorname{ker}\left(\bar{\alpha}_{n}^{*}\right)$ consists of all polynomials $p \in \mathcal{M} \cap \mathcal{P}$ such that for all $1 \leq l \leq n-1$ and all $1 \leq j_{1}<\cdots<j_{l} \leq n-1$ the polynomials $p\left(\cdot ; \lambda_{j_{1}} \cdots \lambda_{j_{l}}\right)$ and $p\left(\cdot ; \lambda_{j_{1}} \cdots \lambda_{j_{l}} \lambda_{n}\right)=\partial_{\lambda_{n}} p\left(\cdot ; \lambda_{j_{1}} \cdots \lambda_{j_{l}}\right)$ belong to $\mathcal{M}$. This implies the result.

\section{Proposition 2.31.}

(a) Let $E_{1}, \ldots, E_{n}$ be finite dimensional $\mathcal{O}_{0}$-modules. There exist $N, k \in$ $\mathbb{N}$ such that $E_{1} \otimes \cdots \otimes E_{n}$ is a quotient of the $\mathcal{O}_{0}$-module $\mathbb{C}^{N} \otimes$ $\mathcal{O}_{0} / \mathcal{M}^{k+1}$ (action on the second tensor component).

(b) Let $\mathcal{I}$ be a cofinite ideal of $\mathcal{O}_{0}$. There exist $\lambda_{1}, \ldots, \lambda_{n} \in \mathfrak{v}_{\mathbb{C}}$ such that the algebra homomorphism

$$
\bar{\alpha}_{n}^{*}: \mathcal{P} \longrightarrow\left(\mathcal{P} / \mathcal{I}_{\lambda_{1}} \cap \mathcal{P}\right) \otimes \cdots \otimes\left(\mathcal{P} / \mathcal{I}_{\lambda_{n}} \cap \mathcal{P}\right)
$$

satisfies $\operatorname{ker} \bar{\alpha}_{n}^{*} \subset \mathcal{I}$. In particular, the $\mathcal{O}_{0}$-module $\mathcal{O}_{0} / \mathcal{I}$ is a subquotient of $\left(\mathcal{O}_{0} / \mathcal{I}_{\lambda_{1}}\right) \otimes \cdots \otimes\left(\mathcal{O}_{0} / \mathcal{I}_{\lambda_{n}}\right)$.

Proof. Assertion (a) follows from Corollary 2.15 and (2.8). For the second assertion, we note that by Lemma 2.17, there exists a number $k \in \mathbb{N}$ such that $(\mathcal{M} \cap \mathcal{P})^{k+1} \subset \mathcal{I} \cap \mathcal{P} \subset \mathcal{M}$. Fix a basis $\left\{X_{1}, \ldots, X_{N}\right\}$ of $\mathfrak{v}$. Put $n:=k N$ and

$$
\lambda_{k j+i}:=X_{j} \quad \text { for } \quad 0 \leq j \leq N-1 \text { and } 1 \leq i \leq k .
$$

By Lemma 2.30, the ideal ker $\bar{\alpha}_{n}^{*}$ is equal to

$$
\left\{p \in \mathcal{M} \cap \mathcal{P} \mid p\left(\cdot ; \lambda_{j_{1}} \cdots \lambda_{j_{l}}\right) \in \mathcal{M}, 1 \leq l \leq n, 1 \leq j_{1}<\cdots<j_{j} \leq n\right\} .
$$

Hence, if $p \in \operatorname{ker} \bar{\alpha}_{n}^{*}$, then

$$
p\left(0 ; X^{\beta}\right)=0, \quad|\beta| \leq k .
$$

This implies that $p \in(\mathcal{M} \cap \mathcal{P})^{k+1}$. Thus, ker $\bar{\alpha}_{n}^{*} \subset \mathcal{I}$. In particular, it follows that the $\mathcal{P}$-module $\mathcal{P} /(\mathcal{I} \cap \mathcal{P})$ is a subquotient of $\left(\mathcal{P} / \mathcal{I}_{\lambda_{1}} \cap \mathcal{P}\right) \otimes \cdots \otimes$ $\left(\mathcal{P} / \mathcal{I}_{\lambda_{n}} \cap \mathcal{P}\right)$. The final assertion now follows by application of Lemmas 2.16 and 2.17 .

2.2. Holomorphic families of continuous representations. We retain our assumption that $\Omega$ is an open subset of the finite dimensional complex linear space $\mathfrak{v}_{\mathbb{C}}$.

If $X$ is a locally compact Hausdorff space, and $V$ a locally convex (Hausdorff) space, then by $C(X, V)$ we denote the space of $V$-valued continuous functions on $X$, equipped with the topology of uniform convergence on compact subsets. If $V$ is quasi-complete, then $C(X, V)$ is quasi-complete as well.

Let $V$ be a quasi-complete locally convex space. The subspace $\mathcal{O}(\Omega, V)$ of $V$-valued holomorphic functions on $\Omega$ is closed in $C(\Omega, V)$ hence quasicomplete of its own right. For our further considerations, it is important to note that the algebraic tensor product $\mathcal{P}\left(\mathfrak{v}_{\mathbb{C}}\right) \otimes V$ is dense in $\mathcal{O}(\Omega, V)$, see Lemma A.1. 
If $V, W$ are locally convex spaces, we write $\operatorname{Hom}(V, W)$ for the space of continuous complex linear maps $V \rightarrow W$. This space, equipped with the strong operator topology is locally convex again. Moreover, if $V$ is barrelled and $W$ quasi-complete, then $\operatorname{Hom}(V, W)$ is quasi-complete as well, see appendix, text before Lemma A.4.

As usual, we write $\operatorname{End}(V)$ for $\operatorname{Hom}(V, V)$. Let $U$ be a third locally convex space. Then the composition map

$$
\beta: \operatorname{Hom}(U, V) \times \operatorname{Hom}(V, W) \rightarrow \operatorname{Hom}(U, W), \quad(A, B) \mapsto B \circ A
$$

is bilinear and separately continuous. Moreover, if $V$ is barrelled, then by the principle of uniform boundedness, $\beta$ is continuous relative to (i.e., when restricted to) sets of the form $\operatorname{Hom}(U, V) \times C$, with $C \subset \operatorname{Hom}(V, W)$ compact; see appendix, Lemma A.4. Now assume that $U, V$ and $W$ are quasi-complete locally convex spaces. If $U$ and $V$ are barrelled, then it follows from the material in the appendix, see Lemma A.6, that the natural pointwise composition defines a bilinear map

$$
\beta_{*}: \mathcal{O}(\Omega, \operatorname{Hom}(U, V)) \times \mathcal{O}(\Omega, \operatorname{Hom}(V, W)) \rightarrow \mathcal{O}(\Omega, \operatorname{Hom}(U, W)) .
$$

Definition 2.32. A holomorphic family of continuous representations of $G$ over $\Omega$ is a pair $(\pi, V)$ such that the following conditions are fulfilled:

(a) $V$ is a Fréchet space.

(b) $\pi$ is a continuous map from $G$ to $\mathcal{O}(\Omega, \operatorname{End}(V))$ satisfying:

(1) $\pi\left(g_{1} g_{2}\right)=\pi\left(g_{1}\right) \pi\left(g_{2}\right), \quad g_{1}, g_{2} \in G$, $\pi\left(e_{G}\right)=1_{\pi}$, where $1_{\pi}(\mu)=\mathrm{id}_{V}$ for all $\mu \in \Omega$;

(2) for every $k \in K$, the $\operatorname{End}(V)$-valued function $\pi(k)$ is constant on $\Omega$.

A holomorphic family of smooth representations of $G$ over $\Omega$ is a family $(\pi, V)$ as above such that in addition

$$
(g, \lambda) \mapsto \pi(g)(\lambda) v, \quad G \times \Omega \rightarrow V
$$

is smooth for every $v \in V$.

Remark 2.33. Given $g \in G$ and $\lambda \in \Omega$ we agree to write $\pi_{\lambda}(g):=\pi(g)(\lambda)$. The condition that $V$ is Fréchet ensures that the principle of uniform boundedness is valid. By application of this principle it follows that the map

$$
G \times \Omega \times V \rightarrow V, \quad(g, \lambda, v) \mapsto \pi_{\lambda}(g) v
$$

is continuous and holomorphic in $\lambda$. More generally we could have given the definition of a holomorphic family of continuous representations under the weaker assumption that $V$ be quasi-complete and barrelled. All results of the present section and the next are in fact valid under this weaker assumption. However, in $\$ 2.4$ the assumption that $V$ is Fréchet will really be needed.

Definition 2.34. Let $\left(\pi, V_{\pi}\right)$ and $\left(\rho, V_{\rho}\right)$ be two holomorphic families of continuous representations of $G$ over $\Omega$. A holomorphic family of intertwining operators, $T$, between $\left(\pi, V_{\pi}\right)$ and $\left(\rho, V_{\rho}\right)$ is an element of $\mathcal{O}\left(\Omega, \operatorname{Hom}\left(V_{\pi}, V_{\rho}\right)\right)$ satisfying:

$$
T \pi(g)=\rho(g) T, \quad g \in G .
$$

Definition 2.35. The category $\mathrm{HF}_{G}$ is defined as follows. 
(a) The objects are the holomorphic families of continuous representations of $G$ over $\Omega$.

(b) The morphisms are the holomorphic families of intertwining operators between two objects.

For any $\left(\pi, V_{\pi}\right) \in \mathrm{HF}_{G}$, the identity morphism is the holomorphic family $1_{\pi}$ of interwining operators defined by:

$$
1_{\pi}(\lambda):=\mathrm{id}_{V_{\pi}}, \quad \lambda \in \Omega
$$

The composition $T^{\prime} \circ T$ of two (composable) morphisms is given by pointwise composition: $\left(T^{\prime} \circ T\right)_{\lambda}=T_{\lambda}^{\prime} \circ T_{\lambda}$. It is again a holomorphic family of intertwining operators, by virtue of Lemma A.6.

If $V$ and $W$ are locally convex spaces, and $E$ a finite dimensional complex linear space, then the map $\left(A_{1}, A_{2}\right) \mapsto A_{1} \otimes A_{2}$ induces a linear isomorphism

$$
\operatorname{End}(E) \otimes \operatorname{Hom}(V, W) \simeq \operatorname{Hom}(E \otimes V, E \otimes W)
$$

which we shall use for identifying these spaces. Accordingly, if $V$ and $W$ are quasi-complete, and $V$ barrelled, then

$$
J^{(E)}: T \mapsto T^{(E)}
$$

defines a continuous linear map

$$
\mathcal{O}(\Omega, \operatorname{Hom}(V, W)) \rightarrow \mathcal{O}(\Omega, \operatorname{Hom}(E \otimes V, E \otimes W))
$$

(see Remark A.7).

Definition 2.36. Let $E$ be a finite dimensional $\mathcal{O}_{0}$-module.

(a) For $(\pi, V) \in \mathrm{HF}_{G}$, we define $\pi^{(E)}$ to be the continuous map

$$
\pi^{(E)}=J^{(E)} \circ \pi: \quad g \mapsto \pi(g)^{(E)}, \quad G \rightarrow \mathcal{O}(\Omega, \operatorname{End}(E \otimes V)) .
$$

(b) For any morphism $T:\left(\pi, V_{\pi}\right) \rightarrow\left(\rho, V_{\rho}\right)$ of $\mathrm{HF}_{G}$, we define

$$
T^{(E)}:=J^{(E)}(T) \in \mathcal{O}\left(\Omega, \operatorname{Hom}\left(E \otimes V_{\pi}, E \otimes V_{\rho}\right)\right) .
$$

Proposition 2.37. Let $E$ be a finite dimensional $\mathcal{O}_{0}$-module. Then $J^{(E)}$ defines a functor from $\mathrm{HF}_{G}$ to itself.

Proof. Let $(\pi, V) \in \mathrm{HF}_{G}$. We will first show that $\left(\pi^{(E)}, E \otimes V\right) \in \mathrm{HF}_{G}$. Let $\beta$ denote the composition map in $\operatorname{End}(V)$. It is a bilinear map, which preserves holomorphy on $\Omega$, by Lemma A.6. Moreover, by Corollary A.12 we have, for all $g_{1}, g_{2} \in G$,

$$
\begin{aligned}
\pi^{(E)}\left(g_{1} g_{2}\right) & =\pi\left(g_{1} g_{2}\right)(E) \\
& =\left(\pi\left(g_{1}\right) \pi\left(g_{2}\right)\right)^{(E)} \\
& =\pi\left(g_{1}\right)^{(E)} \pi\left(g_{2}\right)^{(E)} \\
& =\pi^{(E)}\left(g_{1}\right) \pi^{(E)}\left(g_{2}\right) .
\end{aligned}
$$

Also, $\pi^{(E)}\left(e_{G}\right)=1_{\pi}^{(E)}=1_{\pi^{(E)}}$. For $k \in K$, the $\operatorname{End}(V)$-valued function $\pi(k)$ is a constant on $\Omega$, and therefore, so is the $\operatorname{End}(E \otimes V)$-valued function $\pi^{(E)}(k)=\pi(k)^{(E)}$. Hence (b.1) and (b.2) of Definition 2.32 are fulfilled.

It remains to show that, for any morphism $T:\left(\pi, V_{\pi}\right) \rightarrow\left(\rho, V_{\rho}\right)$ of $\mathrm{HF}_{G}$, $T^{(E)}$ is a morphism of $\mathrm{HF}_{G}$ from $\left(\pi^{(E)}, E \otimes V_{\pi}\right)$ to $\left(\rho^{(E)}, E \otimes V_{\rho}\right)$. For this it suffices to show that, for $g \in G$,

$$
T^{(E)} \pi^{(E)}(g)=\rho_{21}^{(E)}(g) T^{(E)} .
$$


This follows from Corollary A.12, see also the first part of the present proof.

The category $\mathrm{HF}_{G}$ has a null object, $(0,\{0\})$, and one can define a biproduct in $\mathrm{HF}_{G}$ as follows. Let $\left(\pi, V_{\pi}\right),\left(\rho, V_{\rho}\right) \in \mathrm{HF}_{G}$. Set, for any $g \in G, \lambda \in \Omega$ and $(v, w) \in V_{\pi} \oplus V_{\rho}$,

$$
(\pi \oplus \rho)_{\lambda}(g)(v, w):=\left(\pi_{\lambda}(g) v, \rho_{\lambda}(g) w\right) .
$$

Then $\left(\pi \oplus \rho, V_{\pi} \oplus V_{\rho}\right)$ defines an object of $\mathrm{HF}_{G}$.

We define the full subcategory $\mathrm{HF}_{G}^{\infty}$ of $\mathrm{HF}_{G}$ by stipulating that the objects are the holomorphic families of smooth representations over $\Omega$ (the set of morphisms between objects in $\mathrm{HF}_{G}^{\infty}$ coincides with the set of morphisms between the objects viewed as objects for the bigger category $\mathrm{HF}_{G}$. Likewise, the full subcategory $\mathrm{HF}_{G}^{\infty}$,adm of $\mathrm{HF}_{G}^{\infty}$ consists of the objects $(\pi, V)$ of $\mathrm{HF}_{G}^{\infty}$ with $V_{K}$ admissible.

If $(\pi, V) \in \mathrm{HF}_{G}$, then the identity morphism $1_{\pi}$ is constant as a $\operatorname{End}(V)$ valued function on $\Omega$. This enables us to define a particular subcategory.

Definition 2.38. The subcategory ${ }^{\circ} \mathrm{HF}_{G}$ of $\mathrm{HF}_{G}$ is defined as follows.

(a) The objects are the objects of $\mathrm{HF}_{G}$.

(b) If $(\pi, V)$ and $(\rho, W)$ are objects of $\mathrm{HF}_{G}$, then the associated collection of ${ }^{\circ} \mathrm{HF}_{G}$-morphisms consists of all $\mathrm{HF}_{G}$-morphisms $T$ in $\mathcal{O}(\Omega, \operatorname{Hom}(V, W))$ which are constant as a function on $\Omega$.

In a similar fashion, we define the subcategories ${ }^{\circ} \mathrm{HF}_{G}^{\infty}$ and ${ }^{\circ} \mathrm{HF}_{G}^{\infty}$,adm of $\mathrm{HF}_{G}^{\infty}$ and $\mathrm{HF}_{G}^{\infty, a d m}$, respectively.

Note that ${ }^{\circ} \mathrm{HF}_{G}^{\infty}$,adm is a full subcategory of ${ }^{\circ} \mathrm{HF}_{G}^{\infty}$, which in turn is a full subcategory of ${ }^{\circ} \mathrm{HF}_{G}$.

Remark 2.39. For every $E \in \mathrm{FM}_{\mathcal{O}_{0}}$, the functor $J^{(E)}: \mathrm{HF}_{G} \rightarrow \mathrm{HF}_{G}$ leaves all subcategories $\mathrm{HF}_{G}^{\infty}, \mathrm{HF}_{G}^{\infty}$,adm $,{ }^{\circ} \mathrm{HF}_{G},{ }^{\circ} \mathrm{HF}_{G}^{\infty}$ and ${ }^{\circ} \mathrm{HF}_{G}^{\infty}$,adm invariant.

Lemma 2.40. Let $\psi: E \rightarrow E^{\prime}$ be a morphism in $\mathrm{FM}_{\mathcal{O}_{0}}$ and let $\left(\pi, V_{\pi}\right)$ be a holomorphic family of representations. Then $\psi \otimes 1_{\pi}$ intertwines the families $\pi^{(E)}$ and $\pi^{\left(E^{\prime}\right)}$.

Proof. If $f \in \mathcal{O}(\Omega)$, then from the definitions it readily follows that

$$
\psi \circ f^{(E)}(\mu)=f^{\left(E^{\prime}\right)}(\mu) \circ \psi, \quad \mu \in \Omega .
$$

From this and the identification $\operatorname{End}(E) \otimes \operatorname{End}\left(V_{\pi}\right) \simeq \operatorname{End}\left(E \otimes V_{\pi}\right)$, it follows that for all $g \in G$ the map $\psi \otimes 1_{\pi}: E \otimes V_{\pi} \rightarrow E^{\prime} \otimes V_{\pi}$ intertwines $\pi_{\mu}^{(E)}(g)=\pi(g)^{(E)}(\mu)$ with $\pi_{\mu}^{\left(E^{\prime}\right)}(g)=\pi(g)^{\left(E^{\prime}\right)}(\mu)$.

The above lemma justifies the following definition.

Definition 2.41. Given any $\left(\pi, V_{\pi}\right) \in \operatorname{obj}\left(\mathrm{HF}_{G}\right)$, we define the functor $X_{\pi}$ from $\mathrm{FM}_{\mathcal{O}_{0}}$ to ${ }^{\circ} \mathrm{HF}_{G}$ as follows.

(a) For an object $E \in \mathrm{FM}_{\mathcal{O}_{0}}$, the associated object of ${ }^{\circ} \mathrm{HF}_{G}$ is given by

$$
X_{\pi}(E):=\left(\pi^{(E)}, E \otimes V_{\pi}\right) .
$$


(b) For a morphism $\psi: E \rightarrow E^{\prime}$ of $\mathrm{FM}_{\mathcal{O}_{0}}$, the associated morphism of ${ }^{\circ} \mathrm{HF}_{G}$ is given by

$$
X_{\pi}(\psi):=\psi \otimes 1_{\pi}: \quad\left(\pi^{(E)}, E \otimes V_{\pi}\right) \longrightarrow\left(\pi^{\left(E^{\prime}\right)}, E^{\prime} \otimes V_{\pi}\right) .
$$

Remark 2.42. It is readily checked that $X_{\pi}$ is a functor. Indeed, $X_{\pi}$ respects composition of morphisms, and $X_{\pi}\left(\mathrm{id}_{E}\right)=\mathrm{id}_{E \otimes V_{\pi}}=1_{\pi^{(E)}}$.

As $\mathrm{FM}_{\mathcal{O}_{0}}$ is an abelian category, we have the usual notion of finite direct sums and exact sequences in $\mathrm{FM}_{\mathcal{O}_{0}}$.

The category Vect of complex vector spaces is abelian. If $T:(\pi, V) \rightarrow$ $(\rho, W)$ is a morphism in ${ }^{\circ} \mathrm{HF}_{G}$, then there exists a unique linear map $T_{0}: V \rightarrow$ $W$ such that $T(\lambda)=T_{0}$ for all $\lambda \in \Omega$. By abuse of notation we will write $T$ for $T_{0}$. We thus have a forgetful functor ${ }^{\circ} \mathrm{HF}_{G} \rightarrow$ Vect.

The category ${ }^{\circ} \mathrm{HF}_{G}$ is not abelian. Nevertheless, we may use the forgetful functor to define exact sequences

Definition 2.43. A sequence $\left(\left(\pi_{k}, V_{k}\right), T_{k}\right), p \leq k \leq q$, in the category ${ }^{\circ} \mathrm{HF}_{G}$, where $p, q \in \mathbb{Z}, p<q$, will be called exact if its image under the forgetful functor ${ }^{\circ} \mathrm{HF}_{G} \rightarrow$ Vect is exact, i.e., the image of $T_{k-1}$ equals the kernel of $T_{k}$ for all $p<k \leq q$.

Lemma 2.44. Let $\left(\pi, V_{\pi}\right) \in \operatorname{obj}\left(\mathrm{HF}_{G}\right)$. Then the functor $X_{\pi}: \mathrm{FM}_{\mathcal{O}_{0}} \rightarrow$ ${ }^{\circ} \mathrm{HF}_{G}$ has the following properties.

(a) It sends every short exact sequence $0 \rightarrow E \rightarrow E^{\prime} \rightarrow E^{\prime \prime} \rightarrow 0$ to a similar short exact sequence in ${ }^{\circ} \mathrm{HF}_{G}$.

(b) It sends every exact sequence of the form $0 \rightarrow E \rightarrow E^{\prime}$ in $\mathrm{FM}_{\mathcal{O}_{0}}$ to an exact sequence of similar form in ${ }^{\circ} \mathrm{HF}_{G}$.

(c) It sends every exact sequence of the form $E^{\prime} \rightarrow E^{\prime \prime} \rightarrow 0$ in $\mathrm{FM}_{\mathcal{O}_{0}}$ to an exact sequence of similar form in ${ }^{\circ} \mathrm{HF}_{G}$.

(d) It sends a direct sum of the form $E=E_{1} \oplus E_{2}$ in $\mathrm{FM}_{\mathcal{O}_{0}}$ to a similar direct sum in ${ }^{\circ} \mathrm{HF}_{G}$.

Proof. Let FDVect denote the (abelian) category of finite dimensional complex linear spaces. Then we have a forgetful functor $\mathcal{F}$ from $\mathrm{FM}_{\mathcal{O}_{0}}$ to FDVect. A sequence in $\mathrm{FM}_{\mathcal{O}_{0}}$ is exact if and only if its image under $\mathcal{F}$ is exact in FDVect. According to the definition above, the forgetful functor $\mathcal{F}^{\prime}:{ }^{\circ} \mathrm{HF}_{G} \rightarrow$ Vect has a similar property.

Given $U \in$ Vect we define the functor $X_{U}$ : FDVect $\rightarrow$ Vect by $X_{U}(E)=$ $E \otimes U$ for an object $E$ of FDVect. A morphism $f: E \rightarrow E^{\prime}$ in $\mathrm{FM}_{\mathcal{O}_{0}}$ is mapped to $X_{U}(f):=f \otimes \mathrm{id}_{U}: E \otimes U \rightarrow E^{\prime} \otimes U$. It is readily seen that the functor $X_{U}$ is exact, and has the obvious properties analogous to (a) - (d).

Since $\mathcal{F}^{\prime} \circ X_{\pi}=X_{\mathcal{F}^{\prime}(\pi, V)} \circ \mathcal{F}$, assertions (a), (b) and (c) of the lemma follow. For assertion (d) it remains to be shown that each natural embedding $\mathrm{i}_{j}: E_{j} \rightarrow E$, for $j=1,2$, is mapped to an embedding $X_{\pi}\left(\mathrm{i}_{j}\right)$ from $X_{\pi}\left(E_{j}\right)$ onto a closed subspace of $X_{\pi}(E)$. Let $p_{j}: E \rightarrow E_{j}$ be the natural projection. Then by exactness of the sequence $0 \rightarrow E_{1} \rightarrow E \rightarrow E_{2} \rightarrow 0$ it follows from the established assertion (a) that $0 \rightarrow X_{\pi}\left(E_{1}\right) \rightarrow X_{\pi}(E) \rightarrow X_{\pi}\left(E_{2}\right) \rightarrow 0$ is exact. This implies that $X_{\pi}\left(\mathrm{i}_{1}\right)$ has closed image in $X_{\pi}(E)$. Likewise, $X_{\pi}\left(\mathrm{i}_{2}\right)$ is seen to have close image.

Remark 2.45. In view of Remark 2.39, Lemma 2.44 has an obvious generalization to objects from $\mathrm{HF}_{G}^{\infty}$ and from $\mathrm{HF}_{G}^{\infty, a d m}$. 
Proposition 2.46. (a) Let $E_{1}, \ldots, E_{n} \in \mathrm{FM}_{\mathcal{O}_{0}}$ and set $E:=E_{1} \otimes \cdots \otimes$ $E_{n}$. Then there exist $N, k \in \mathbb{N}$ such that, for any object $\left(\pi, V_{\pi}\right)$ in $\mathrm{HF}_{G}$ (resp. $\left.\mathrm{HF}_{G}^{\infty}, \mathrm{HF}_{G}^{\infty, \mathrm{adm}}\right)$, the family $\left(\pi^{(E)}, E \otimes V_{\pi}\right)$ is a quotient of

$$
\left(\mathrm{id}_{\mathbb{C}^{N}} \otimes \pi^{\left(\mathcal{O}_{0} / \mathcal{M}^{k+1}\right)}, \mathbb{C}^{N} \otimes\left(\mathcal{O}_{0} / \mathcal{M}^{k+1} \otimes V_{\pi}\right)\right)
$$

in the category ${ }^{\circ} \mathrm{HF}_{G}$ (resp. ${ }^{\circ} \mathrm{HF}_{G}^{\infty},{ }^{\circ} \mathrm{HF}_{G}^{\infty}$,adm $)$.

(b) Let $E \in \mathrm{FM}_{\mathcal{O}_{0}}$. Then there exist $\lambda_{1}, \ldots, \lambda_{n} \in \mathfrak{v}_{\mathbb{C}}$ such that, for any object $\left(\pi, V_{\pi}\right)$ in $\mathrm{HF}_{G}$ (resp. $\mathrm{HF}_{G}^{\infty}, \mathrm{HF}_{G}^{\infty}$,adm $)$, the family $\left(\pi^{(E)}, E \otimes\right.$ $\left.V_{\pi}\right)$ is a subquotient of

$$
\left(\pi^{\left(\left(\mathcal{O}_{0} / \mathcal{I}_{\lambda_{1}}\right) \otimes \cdots \otimes\left(\mathcal{O}_{0} / \mathcal{I}_{\lambda_{n}}\right)\right)},\left(\mathcal{O}_{0} / \mathcal{I}_{\lambda_{1}}\right) \otimes \cdots \otimes\left(\mathcal{O}_{0} / \mathcal{I}_{\lambda_{n}}\right) \otimes V_{\pi}\right)
$$

in the category ${ }^{\circ} \mathrm{HF}_{G}$ (resp. ${ }^{\circ} \mathrm{HF}_{G}^{\infty},{ }^{\circ} \mathrm{HF}_{G}^{\infty}$,adm $)$.

Proof. This follows from Proposition 2.31 combined with Lemma 2.44.

2.3. Holomorphic families of admissible $(\mathfrak{g}, K)$-modules. For the purpose of this paper, it is convenient to introduce the following notion of holomorphic families of admissible $(\mathfrak{g}, K)$-modules. Recall that $\Omega$ is an open subset of the finite dimensional complex linear space $\mathfrak{v}_{\mathbb{C}}$.

Definition 2.47. A holomorphic family of admissible $(\mathfrak{g}, K)$-modules over $\Omega$ is a triple $\left(\pi_{1}, \pi_{2}, V\right)$ satisfying the following conditions.

(a) $V$ is a complex vector space.

(b) $\pi_{1}$ is a map from $U(\mathfrak{g}) \times \Omega$ to $\operatorname{End}(V)$ such that

(1) for each $\lambda \in \Omega$, the map $\pi_{1}(\cdot, \lambda)$ is a Lie algebra homomorphism;

(2) for each $u \in U(\mathfrak{g})$ and every $v \in V$, the vector subspace of $V$ generated by the $\pi_{1}(u, \lambda) v, \lambda \in \Omega$, is finite dimensional and the map $\lambda \mapsto \pi_{1}(u, \lambda) v$ is holomorphic from $\Omega$ into this subspace.

(c) $\pi_{2}$ is a Lie group homomorphism from $K$ to $\mathrm{GL}(V)$ such that:

(1) for each $v \in V$, the vector subspace of $V$ generated by the $\pi_{2}(k) v, k \in K$, is finite dimensional;

(2) for all $v \in V, \lambda \in \Omega, u \in U(\mathfrak{g}), k \in K$ and $X \in \mathfrak{k}$,

$$
\begin{aligned}
\pi_{1}(\operatorname{Ad}(k) u, \lambda) v & =\pi_{2}(k) \pi_{1}(u, \lambda) v, \\
\frac{d}{d t}\left[\pi_{2}(\exp (t X)) v\right]_{\mid t=0} & =\pi_{1}(X, \lambda) v ;
\end{aligned}
$$

(d) for every $\delta \in \hat{K}$, the $K$-isotypic component $\pi_{2, \delta}$ of $\pi_{2}$ of type $\delta$ is finite dimensional.

Remark 2.48. Let $\left(\pi_{1}, \pi_{2}, V\right)$ be as in the above definition. Given $\lambda \in \Omega$ we agree to write $\pi_{1 \lambda}$ for the map $\pi_{1}(\cdot, \lambda): U(\mathfrak{g}) \rightarrow \operatorname{End}(V)$. Then $\left(\pi_{1 \lambda}, \pi_{2}, V\right)$ is an admissible $(\mathfrak{g}, K)$-module.

We also need the following notion of holomorphic family of intertwining operators.

Definition 2.49. Let $\left(\pi_{1}, \pi_{2}, V\right)$ and $\left(\rho_{1}, \rho_{2}, W\right)$ be two holomorphic families of admissible $(\mathfrak{g}, K)$-modules over $\Omega$. A holomorphic family of intertwining operators between $\left(\pi_{1}, \pi_{2}, V\right)$ and $\left(\rho_{1}, \rho_{2}, W\right)$ is a function $T: \Omega \rightarrow$ $\operatorname{Hom}(V, W)$ satisfying the following conditions.

(a) $T(\lambda) \pi_{1}(u, \lambda)=\rho_{1}(u, \lambda) T(\lambda)$, for all $\lambda \in \Omega$ and $u \in U(\mathfrak{g})$;

(b) $T(\lambda) \pi_{2}(k)=\rho_{2}(k) T(\lambda)$, for all $\lambda \in \Omega$ and $k \in K$; 
(c) for any finite dimensional subspace $\tilde{V}$ of $V$, there exists a finite dimensional subspace $\tilde{W}$ of $W$ such that $T(\lambda)(\tilde{V}) \subset \tilde{W}$ for all $\lambda \in \Omega$, and the associated function $\left.\lambda \mapsto T(\lambda)\right|_{\tilde{V}}$ belongs to $\mathcal{O}(\Omega, \operatorname{Hom}(\tilde{V}, \tilde{W}))$.

Let $\mathrm{HF}_{(\mathfrak{g}, K)}^{\mathrm{adm}}$ denote the corresponding category of holomorphic families of admissible $(\mathfrak{g}, K)$-modules.

Let $(\pi, V)$ be an object in the category $\mathrm{HF}_{G}^{\infty, \text { adm }}$. Then for each $\lambda \in \Omega$, $u \in U(\mathfrak{g})$ and $v \in V$ we may define

$$
\pi(u)(\lambda) v:=\left.L_{u^{\vee}}(g \mapsto \pi(g)(\lambda) v)\right|_{g=e} .
$$

We put $\pi_{1}(u, \lambda)=\left.\pi(u)(\lambda)\right|_{V_{K}}$ and $\pi_{2}(k)=\left.\pi(k)\right|_{V_{K}}$.

Lemma 2.50. Let $(\pi, V) \in \mathrm{HF}_{G}^{\infty, a d m}$ and let $\pi_{1}, \pi_{2}$ be defined as above. Then

$$
(\pi, V)_{K}:=\left(\pi_{1}, \pi_{2}, V_{K}\right)
$$

is a holomorphic family in $\mathrm{HF}_{(\mathfrak{g}, K)}^{\mathrm{adm}}$. Moreover, $(\cdot)_{K}:(\pi, V) \mapsto(\pi, V)_{K}$ defines a functor $\mathrm{HF}_{G}^{\infty, \mathrm{adm}} \rightarrow \mathrm{HF}_{(\mathfrak{g}, K)}^{\mathrm{adm}}$.

Proof. It follows from the smoothness of the map (2.17), the continuity of the map $L_{u^{\vee}}: C^{\infty}(G) \rightarrow C^{\infty}(G)$ and the holomorphy with respect to $\lambda$, that $\varphi: \lambda \mapsto \pi(u)(\lambda) v$ defines a holomorphic function $\Omega \rightarrow V$. Let now $v \in V_{K}$ and let $\vartheta_{2} \subset \widehat{K}$ denote the set of $K$-types appearing in the $\pi(K)$-span of $v$. Moreover, let $\vartheta_{1}$ be the set of $K$-types which appear in the $\operatorname{Ad}(K)$-span of $u$. Finally, let $\vartheta$ be the union of the sets of $K$-types of $\delta_{1} \otimes \delta_{2}$, for $\delta_{j} \in \vartheta_{j}$, $j=1,2$. Then $\varphi$ has image contained in the finite dimensional subspace $V_{\vartheta} \subset V_{K}$. It follows that $\varphi$ is holomorphic as a function $\Omega \rightarrow V_{\vartheta}$. This shows that $\left(\pi_{1}, \pi_{2}, V_{K}\right)$ satisfies condition (b)(2) of Definition 2.47. The other conditions of that definition are pointwise in $\lambda$, and therefore consequences of the standard theory of assigning the $(\mathfrak{g}, K)$-module of $K$-finite vectors to an admissible smooth Fréchet representation, see for instance [9, Lemma 3.3.5]. The latter assignment is a functor from the category of admissible smooth Fréchet representations to the category of Harish-Chandra modules. This implies that $(\pi, V) \mapsto(\pi, V)_{K}$ has functorial properties which are pointwise in $\lambda$. This in turn is readily seen to imply that $(\cdot)_{K}$ is a functor as stated.

We will now discuss the functor $J^{(E)}$ on the level of holomorphic families of admissible $(\mathfrak{g}, K)$-modules.

Definition 2.51. Let $E \in \mathrm{FM}_{\mathcal{O}_{0}}$. For any $\left(\pi_{1}, \pi_{2}, V\right) \in \mathrm{HF}_{(\mathfrak{g}, K)}^{\text {adm }}$ the triple ${ }_{\left(J^{(E)}\right.} \pi_{1}, J^{(E)} \pi_{2}, J^{(E)} V$ ) (also denoted $\left(\pi_{1}^{(E)}, \pi_{2}^{(E)}, V^{(E)}\right)$ ) is defined as follows.

(a) $J^{(E)} V:=E \otimes V$.

(b) $J^{(E)} \pi_{1}$ is the map from $U(\mathfrak{g}) \times \Omega$ to $\operatorname{End}(E \otimes V)$ given by

$$
J^{(E)} \pi_{1}(u, \lambda) e \otimes v:=\left(\pi_{1}(u, \cdot) v\right)^{(E)}(\lambda) e,
$$

for $u \in U(\mathfrak{g}), \lambda \in \Omega, e \in E$, and $v \in V$.

(c) $J^{(E)} \pi_{2}:=\operatorname{id}_{E} \otimes \pi_{2}$.

We now have functors $J^{(E)}$ on $\mathrm{HF}_{G}^{\infty, \text { adm }}$ and $\mathrm{HF}_{(\mathfrak{g}, K)}^{\mathrm{adm}}$. They are linked as follows. 


\section{Lemma 2.52.}

(a) For any $E \in \mathrm{FM}_{\mathcal{O}_{0}}$, the assignment $J^{(E)}$ defines a functor from $\mathrm{HF}_{(\mathfrak{g}, K)}^{\mathrm{adm}}$ to itself.

(b) The following diagram commutes:

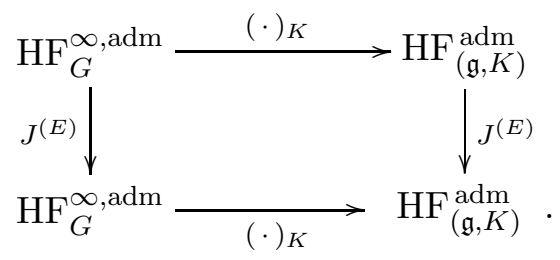

Proof. Assertion (a) follows by similar arguments as in the proof of Proposition 2.37, For (b), assume that $(\pi, V)$ is a family in $\mathrm{HF}_{G}^{\infty, \text { adm }}$. Put $(\pi, V)_{K}=$ $\left(\pi_{1}, \pi_{2}, V_{K}\right)$, then

$$
J^{(E)}\left((\pi, V)_{K}\right)=\left(\pi_{1}^{(E)}, \pi_{2}^{(E)},\left(V_{K}\right)^{(E)}\right) .
$$

On the other hand, $J^{(E)}(\pi, V)=\left(\pi^{(E)}, V^{(E)}\right)$ and

$$
\left(J^{(E)}(\pi, V)\right)_{K}=\left(\left(\pi^{(E)}\right)_{1},\left(\pi^{(E)}\right)_{2},\left(V^{(E)}\right)_{K}\right)
$$

Now $V^{(E)}=E \otimes V$ is equipped with the $K$-action on the second component, so that $\left(V^{(E)}\right)_{K}=E \otimes V_{K}=\left(V_{K}\right)^{(E)}$. Moreover, $\pi_{2}^{(E)}(k)=1_{E} \otimes \pi_{2}(k)=$ $\left.1_{E} \otimes \pi(k, \lambda)\right|_{V_{K}}=\left.\pi^{(E)}(k, \lambda)\right|_{E \otimes V_{K}}=\left(\pi^{(E)}\right)_{2}(k)$. It remains to establish the identity

$$
\pi_{1}^{(E)}=\left(\pi^{(E)}\right)_{1}
$$

Since both are representations of $U(\mathfrak{g})$ in $E \times V_{K}$, it suffices to check the identity on a fixed element $X \in \mathfrak{g}$. Fix $e \in E$ and $v \in V$. We first observe that

$$
\pi_{1}^{(E)}(X) v=\left(\pi_{1}(X, \cdot) v\right)^{(E)}=\left.\left[\frac{d}{d t} \pi(\exp t X)(\cdot) v\right]^{(E)}\right|_{t=0} .
$$

In view of the natural identification $\operatorname{End}(E) \otimes V \simeq \operatorname{Hom}(E, E \otimes V)$, we note that

$$
\begin{aligned}
\left(\pi^{(E)}\right)_{1}(X, \cdot) v & =\left.\frac{d}{d t}\left[\pi^{(E)}(\exp t X)(\cdot) v\right]\right|_{t=0} \\
& =\left.\frac{d}{d t}\left[(\pi(\exp t X)(\cdot) v)^{(E)}\right]\right|_{t=0} .
\end{aligned}
$$

The identity (2.20) now follows by application of the lemma below.

Lemma 2.53. Let $V$ be a quasi-complete locally convex space, and let $\varphi: \mathbb{R} \times$ $\Omega \rightarrow V$ be a $C^{1}$-map which is holomorphic in the second variable. Then

$$
\frac{d}{d t}\left[\varphi(t, \cdot)^{(E)}\right]=\left[\frac{\partial \varphi}{\partial t}(t, \cdot)\right]^{(E)} .
$$

Proof. Let $S$ be the space of $C^{1}$-maps $\mathbb{R} \times \Omega \rightarrow V$, equipped with the usual quasi-complete topology. Let $S_{0}$ be the subspace consisting of functions in $S$ which are holomorphic in the second variable. Then $S_{0}$ is closed in $S$, hence quasi-complete. The identity (2.21) at $(t, \lambda)$ can be viewed as an identity of 
continuous linear functionals on $S_{0}$. Hence, it suffices to check the identity on the dense subspace $C^{1}(\mathbb{R}) \otimes \mathcal{O}(\Omega) \otimes V$. This amounts to checking whether

$$
\left(\frac{d}{d t} \otimes I \otimes I\right) \circ\left(I \otimes J^{(E)} \otimes I\right)=\left(I \otimes J^{(E)} \otimes I\right) \circ\left(\frac{d}{d t} \otimes I \otimes I\right) .
$$

The latter is obvious.

2.4. Parabolic induction. Let $\mathfrak{g}=\mathfrak{k} \oplus \mathfrak{p}$ be a Cartan decomposition associated with the maximal compact subgroup $K$ and let $\theta$ be the associated involution of $G$. Let $\mathfrak{a} \subset \mathfrak{p}$ be a maximal abelian subspace, and let $A=\exp \mathfrak{a}$. Let $\mathcal{P}(A)$ denote the collection of parabolic subgroups of $G$ containing $A$. Let $\mathcal{L}(A)$ denote the collection of $\theta$-stable Levi components of parabolic subgroups from $\mathcal{P}(A)$.

Let $\mathfrak{v}$ be a finite dimensional real linear space, and $\Omega$ an open subset of its complexification. For $L \in \mathcal{L}(A)$ we denote by $\mathrm{HF}_{L}$ the category defined as in Definition 2.32, with the group $L$ in place of $G$.

The parabolic induction functor from $\mathrm{HF}_{L}^{\infty}$ to $\mathrm{HF}_{G}^{\infty}$ is defined as follows. Let $\left(\xi, V_{\xi}\right) \in \mathrm{HF}_{L}^{\infty}$ be a holomorphic family of smooth representations of $L$ defined over $\Omega$. Denote by $\bar{\pi}_{P, \xi_{\lambda}}$ the right regular representation of $G$ on $C^{\infty}\left(G: P: \xi_{\lambda}\right)$, where

$$
\begin{aligned}
C^{\infty}\left(G: P: \xi_{\lambda}\right):=\left\{\psi \in C^{\infty}\left(G, V_{\xi}\right):\right. & \psi(n m g)=\xi_{\lambda}(m) \psi(g), \\
& \left.(g, m, n) \in G \times L \times N_{P}\right\} .
\end{aligned}
$$

Let

$$
\begin{aligned}
C^{\infty}(K: \xi):=\left\{\psi \in C^{\infty}\left(K, V_{\xi}\right):\right. & \psi(m k)=\xi(m) \psi(k), \\
& (k, m) \in K \times(K \cap L)\} .
\end{aligned}
$$

Restriction of functions to $K$ induces a continuous linear isomorphism between these spaces. Let $\pi_{P, \xi_{\lambda}}$ denote the representation of $G$ on $C^{\infty}(K: \xi)$, obtained from $\bar{\pi}_{P, \xi_{\lambda}}$ by transfer of structure, and set, for $g \in G, \lambda \in \mathfrak{v}_{\mathbb{C}}$,

$$
\pi_{P, \xi, \lambda}(g):=\pi_{P, \xi_{\lambda}}(g) .
$$

Then it is readily seen that $\left(\pi_{P, \xi}, C^{\infty}(K: \xi)\right)$ belongs to $\mathrm{HF}_{G}^{\infty}$. Here, the information that $V_{\xi}$ is a Fréchet space is needed to conclude that $C^{\infty}(K: \xi)$ is Fréchet, in particular barrelled.

Let $W_{1}, W_{2}$ and $W_{3}$ be quasi-complete locally convex spaces, and let $\alpha$ be a continuous linear map from $W_{2}$ to $W_{3}$. Then the map

$$
L_{\alpha}: \varphi \longmapsto \alpha \circ \varphi, \quad \operatorname{Hom}\left(W_{1}, W_{2}\right) \longrightarrow \operatorname{Hom}\left(W_{1}, W_{3}\right)
$$

is continuous linear. It readily follows that the map

$$
\operatorname{id}_{\mathcal{O}(\Omega)} \widehat{\otimes} L_{\alpha}: \quad \mathcal{O}\left(\Omega, \operatorname{Hom}\left(W_{1}, W_{2}\right)\right) \longrightarrow \mathcal{O}\left(\Omega, \operatorname{Hom}\left(W_{1}, W_{3}\right)\right)
$$

given by $f \mapsto L_{\alpha} \circ f$ is continuous linear. The notation for this map is explained by the fact that it may be viewed as the unique continuous linear extension of $\operatorname{id}_{\mathcal{O}(\Omega)} \otimes L_{\alpha}$.

Lemma 2.54. Let $L_{\alpha}$ be as above and let $E$ be a finite dimensional $\mathcal{O}_{0}$ module. Then we have the following identity of maps

$$
\begin{gathered}
\mathcal{O}\left(\Omega, \operatorname{Hom}\left(W_{1}, W_{2}\right)\right) \longrightarrow \mathcal{O}\left(\Omega, \operatorname{End}(E) \otimes \operatorname{Hom}\left(W_{1}, W_{3}\right)\right): \\
J^{(E)} \circ\left(\operatorname{id}_{\mathcal{O}(\Omega)} \widehat{\otimes} L_{\alpha}\right)=\left(\operatorname{id}_{\mathcal{O}(\Omega)} \widehat{\otimes} \operatorname{id}_{\operatorname{End}(E)} \widehat{\otimes} L_{\alpha}\right) \circ J^{(E)} .
\end{gathered}
$$


Proof. By continuity of the expressions on both sides of the identity it suffices to prove the identity on the dense subspace $\mathcal{O}(\Omega) \otimes \operatorname{Hom}\left(W_{1}, W_{2}\right)$ of $\mathcal{O}\left(\Omega, \operatorname{Hom}\left(W_{1}, W_{2}\right)\right)$. But then the identity becomes obvious, in view of Remark A.7, last line.

The following result may be phrased as 'derivation commutes with induction'.

Proposition 2.55. Let $E$ be a finite dimensional $\mathcal{O}_{0}$-module, $P$ a parabolic subgroup with Levi component $L$, and $\left(\xi, V_{\xi}\right) \in \mathrm{HF}_{L}^{\infty}$. Then

$$
\pi_{P, \xi}^{(E)}=\pi_{P, \xi^{(E)}} .
$$

Proof. Let $g \in G$ and $k \in K$. Put $\alpha(k):=\mathrm{ev}_{k}: C^{\infty}(K: \xi) \rightarrow V_{\xi}$. Let

$$
L_{\alpha(k)}: \operatorname{End}\left(C^{\infty}(K: \xi)\right) \rightarrow \operatorname{Hom}\left(C^{\infty}(K: \xi), V_{\xi}\right)
$$

be defined as in the previous lemma. Accordingly,

$$
\left(\operatorname{id}_{\mathcal{O}(\Omega)} \widehat{\otimes} \operatorname{id}_{E} \widehat{\otimes} L_{\alpha(k)}\right)\left(\pi_{P, \xi}^{(E)}(g)\right)=\left(\left(\operatorname{id}_{\mathcal{O}(\Omega)} \widehat{\otimes} L_{\alpha(k)}\right)\left(\pi_{P, \xi}(g)\right)\right)^{(E)} .
$$

Write $g=n p(g) \kappa(g)$ uniquely via the decomposition $G=N_{P} A_{P}\left(M_{P} \cap\right.$ $\exp \mathfrak{p}) K$, where $n \in N_{P}, p(g) \in A_{P}(M \cap \exp \mathfrak{p})$ and $\kappa(g) \in K$. We then have the following identity:

$$
\left(\operatorname{id}_{\mathcal{O}(\Omega)} \widehat{\otimes} L_{\alpha(k)}\right)\left(\pi_{P, \xi}(g)\right)=\xi(p(k g)) \circ L_{\alpha(\kappa(k g))} .
$$

Hence, it follows from (2.23) that

$$
\begin{aligned}
\left(\operatorname{id}_{\mathcal{O}(\Omega)}\right. & \left.\widehat{\otimes} \operatorname{id}_{E} \widehat{\otimes} L_{\alpha(k)}\right)\left(\pi_{P, \xi}^{(E)}(g)\right) \\
& =\left(\xi(p(k g)) \circ L_{\alpha(\kappa(k g))}\right) \\
& =\left(\xi^{(E)}(p(k g)) \circ L_{\alpha(\kappa(k g))}\right) \\
& =\left(\operatorname{id}_{\mathcal{O}(\Omega)} \widehat{\otimes i d}_{E} \widehat{\otimes} L_{\alpha(k)}\right)\left(\pi_{P, \xi^{(E)}}(g)\right)
\end{aligned}
$$

and the statement follows.

\section{The Arthur-Campoli Relations}

Given a parabolic subgroup $P \in \mathcal{P}(A)$ we denote its Langlands decomposition by $P=M_{P} A_{P} N_{P}$.

Let $\left(\xi, V_{\xi}\right)$ be a smooth, irreducible and admissible Fréchet representation of $M_{P}$. Given $\lambda \in \mathfrak{a}_{P \mathbb{C}}^{*}$ we denote by $\xi \otimes \lambda$ the smooth representation of $L_{P}:=M_{P} A_{P}$ in $V_{\xi}$ defined by

$$
\xi \otimes \lambda(m a)=a^{\lambda+\rho_{P}} \xi(m) .
$$

As usual, here $\rho_{P} \in \mathfrak{a}_{P}^{*}$ is defined by $\rho_{P}=\frac{1}{2} \operatorname{tr}\left(\operatorname{ad}(\cdot) \mid \mathfrak{n}_{P}\right)$. The associated induced representation $\bar{\pi}_{P, \xi \otimes \lambda}$ of $G$ in $C^{\infty}(G: P: \xi \otimes \lambda)$ is defined as in Section 2.4, with $\mathfrak{v}=\mathfrak{a}^{*}, \Omega=\mathfrak{a}_{\mathbb{C}}^{*}$ and $\xi_{\lambda}=\xi \otimes \lambda$. The family of these representations may be viewed as a holomorphic family $\pi_{P, \xi \otimes(\cdot)}$ of smooth representations of $G$ over $\Omega=\mathfrak{a}_{\mathbb{C}}^{*}$ on the fixed space $C^{\infty}(K: \xi)$, defined in (2.22). As in the mentioned section, we agree to write $\pi_{P, \xi, \lambda}=\pi_{P, \xi \otimes \lambda}$. If $P$ is a minimal parabolic subgroup, the representations $\pi_{P, \xi, \lambda}$ just defined are called representations of the smooth minimal principal series of $G$. 
Let now $P \in \mathcal{P}(A)$ be arbitrary again. Then $M_{P}$ is a group of the HarishChandra class, with maximal compact subgroup $K_{P}:=M_{P} \cap K$. Two continuous admissible $M_{P}$-representations of finite length in a quasi-complete locally convex space are said to be infinitesimally equivalent if their HarishChandra modules are equivalent as $\left(\mathfrak{m}_{P}, K_{P}\right)$-modules. For each equivalence class $\omega$ of irreducible unitary representations of $M_{P}$, we fix a smooth admissible Fréchet representation $\xi=\xi_{\omega}$ which is infinitesimally equivalent to a representation of class $\omega$, and which is topologically equivalent to a closed subrepresentation of a representation of the smooth minimal principal series of $M_{P}$. Indeed, this is possible by the subrepresentation theorem for the group $M_{P}$. The set of all these chosen representations $\xi_{\omega}$ is denoted by $M_{P}^{\wedge}$. Thus, $\omega \mapsto \xi_{\omega}$ defines a bijection from the set of equivalence classes of irreducible unitary representations of $M_{P}$ onto $M_{P}^{\wedge}$.

Remark 3.1. In view of the theory of the Casselman-Wallach globalization functor, see [10, Section 11], the representation $\xi_{\omega}$ is a smooth Fréchet globalization of moderate growth of the Harish-Chandra module of any representative of $\omega$. This characterization makes the choice of $M_{P}^{\wedge}$ more natural, but will not be needed in the present paper.

We denote by $M_{P, d s}^{\wedge}$ the subset of $M_{P}^{\wedge}$ consisting of the representations $\xi_{\omega}$, with $\omega$ a discrete series representation. In particular, if $P \in \mathcal{P}(A)$ is minimal, then $M_{P}$ equals the centralizer $M$ of $\mathfrak{a}$ in $K$, and $M_{d s}^{\wedge}=M^{\wedge}$. For each $\left(\xi, V_{\xi}\right) \in M_{P, d s}^{\wedge}$ we put

$$
\mathcal{S}(P, \xi):=\operatorname{End}\left(C^{\infty}(K: \xi)\right)_{K \times K},
$$

and we define the algebraic direct sum of linear spaces

$$
\mathcal{S}(P):=\oplus_{\xi \in \widehat{M}_{P, d s}} \mathcal{S}(P, \xi) .
$$

3.1. The Arthur-Campoli relations. Fix a minimal parabolic subgroup $P_{0}$ in $\mathcal{P}(A)$ and let $P_{0}=M A N_{0}$ be its Langlands decomposition. In [1, III, $\S 4]$, Arthur defines a Paley-Wiener space involving all minimal parabolic subgroups containing $A$. This definition is given in terms of on the one hand Paley-Wiener growth conditions and on the other the so-called ArthurCampoli conditions. In [2, Thm. 3.6] it is shown that the Arthur PaleyWiener space is isomorphic to one defined in terms of the single minimal parabolic subgroup $P_{0}$. We shall now describe the Arthur-Campoli relations in the context of the operator valued Fourier transform $f \mapsto \hat{f}\left(P_{0}\right)$.

For $f \in C_{c}^{\infty}(G, K)$ and $\xi \in M^{\wedge}$, the Fourier transform $\hat{f}\left(P_{0}, \xi\right) \in \mathcal{O}\left(\mathfrak{a}_{\mathbb{C}}^{*}\right) \otimes$ $\mathcal{S}\left(P_{0}, \xi\right)$ is defined by

$$
\hat{f}\left(P_{0}, \xi, \lambda\right):=\int_{G} f(x) \pi_{P_{0}, \xi, \lambda}(x) d x, \quad \lambda \in \mathfrak{a}_{\mathbb{C}}^{*} .
$$

Then $f \mapsto \hat{f}\left(P_{0}\right)$ maps $C_{c}^{\infty}(G, K)$ into $\mathcal{O}\left(\mathfrak{a}_{\mathbb{C}}^{*}\right) \otimes \mathcal{S}\left(P_{0}\right)$.

We define $\Sigma$ to be the set of 4 -tuples of the form $(\xi, \psi, \lambda, u)$ with $\xi \in M^{\wedge}$, $\psi \in \mathcal{S}\left(P_{0}, \xi\right)_{K \times K}^{*}, \lambda \in \mathfrak{a}_{\mathbb{C}}^{*}$ and $u \in S\left(\mathfrak{a}^{*}\right)$. An Arthur-Campoli sequence in $\Sigma$ is defined to be a finite family $\left(\xi_{i}, \psi_{i}, \lambda_{i}, u_{i}\right)$ in $\Sigma$ such that

$$
\sum_{i}\left\langle\pi_{P_{0}, \xi_{i}, \lambda_{i} ; u_{i}}(x), \psi_{i}\right\rangle=0, \quad x \in G .
$$


By integration over $x$ it follows that this condition is equivalent to the condition that

$$
\sum_{i}\left\langle\hat{f}\left(P_{0}, \xi_{i}, \lambda_{i} ; u_{i}\right), \psi_{i}\right\rangle=0, \quad f \in C_{c}^{\infty}(G, K) .
$$

Definition 3.2. A function $\varphi \in \mathcal{O}\left(\mathfrak{a}_{\mathbb{C}}^{*}\right) \otimes \mathcal{S}\left(P_{0}\right)$ is said to satisfy the ArthurCampoli relations if

$$
\sum_{i}\left\langle\varphi_{\xi_{i}, \lambda_{i} ; u_{i}}, \psi_{i}\right\rangle=0
$$

for any Arthur-Campoli sequence $\left(\xi_{i}, \psi_{i}, \lambda_{i}, u_{i}\right)$ in $\Sigma$.

3.2. Reformulation of the Arthur-Campoli relations. In the following, $\mathcal{O}_{0}$ denotes the ring of germs at 0 of holomorphic functions defined on a neighborhood of 0 in $\mathfrak{a}_{\mathbb{C}}^{*}$.

Let $E$ be a finite dimensional $\mathcal{O}_{0}$-module and let $\Xi \subset M^{\wedge}$ and $\Lambda \subset \mathfrak{a}_{\mathbb{C}}^{*}$ be finite sets. We define the representation $\pi_{E, \Xi, \Lambda}$ of $G$ by

$$
\pi_{E, \Xi, \Lambda}=\oplus_{(\xi, \lambda) \in \Xi \times \Lambda} \pi_{P_{0}, \xi, \lambda}^{(E)} .
$$

Note that this representation is admissible and of finite length. Its underlying space is given by

$$
V_{E, \Xi, \Lambda}=\oplus_{(\xi, \lambda) \in \Xi \times \Lambda} \quad E \otimes C^{\infty}(K: \xi) .
$$

For each element $\varphi \in \mathcal{O}\left(\mathfrak{a}_{\mathbb{C}}^{*}\right) \otimes \mathcal{S}\left(P_{0}\right)$ we define the $K \times K$-finite endomorphism $\varphi_{E, \Xi, \Lambda}$ of $V_{E, \Xi, \Lambda}$ by taking the similar direct sum

$$
\varphi_{E, \Xi, \Lambda}:=\oplus_{(\xi, \lambda) \in \Xi \times \Lambda} \varphi_{\xi}^{(E)}(\lambda) .
$$

We note that $\pi_{E, \Xi, \Lambda}\left(C_{c}^{\infty}(G, K)\right)$ is a subset of the space $\operatorname{End}\left(V_{E, \Xi, \Lambda}\right)$ and agree to write $\pi_{E, \Xi, \Lambda}\left(C_{c}^{\infty}(G, K)\right)^{\perp}$ for its annihilator in the space

$$
\operatorname{End}\left(V_{E, \Xi, \Lambda}\right)_{K \times K}^{*} \cdot
$$

Proposition 3.3. Let $\phi \in \mathcal{O}\left(\mathfrak{a}_{\mathbb{C}}^{*}\right) \otimes \mathcal{S}\left(P_{0}\right)$. Then the following conditions are equivalent:

(a) $\phi$ satisfies the Arthur-Campoli relations;

(b) for every finite dimensional $\mathcal{O}_{0}$-module $E$, every pair of finite sets $\Xi \subset M^{\wedge}, \Lambda \subset \mathfrak{a}_{\mathbb{C}}^{*}$ and all $\Psi \in \pi_{E, \Xi, \Lambda}\left(C_{c}^{\infty}(G, K)\right)^{\perp}$,

$$
\left\langle\phi_{E, \Xi, \Lambda}, \Psi\right\rangle=0 \text {. }
$$

We prove the result through a number of lemmas. In the following results a complication is caused by the circumstance that $\operatorname{End}\left(V_{E, \Xi, \Lambda}\right)_{K \times K}$ is not the direct sum of the spaces $\operatorname{End}\left(V_{E, \xi, \lambda}\right)_{K \times K}$, but rather that of the spaces $\operatorname{Hom}\left(V_{E, \xi_{1}, \lambda_{1}}, V_{E, \xi_{2}, \lambda_{2}}\right)_{K \times K}$, for $\xi_{1}, \xi_{2} \in \Xi$ and $\lambda_{1}, \lambda_{2} \in \Lambda$. For $(\xi, \lambda) \in \Xi \times \Lambda$, let

$$
\mathrm{i}_{\xi, \lambda}: \operatorname{End}\left(V_{E, \xi, \lambda}\right)_{K \times K} \longrightarrow \operatorname{End}\left(V_{E, \Xi, \Lambda}\right)_{K \times K}
$$

denote the associated embedding, and let

$$
\operatorname{pr}_{\xi, \lambda}: \operatorname{End}\left(V_{E, \Xi, \Lambda}\right)_{K \times K} \longrightarrow \operatorname{End}\left(V_{E, \xi, \lambda}\right)_{K \times K}
$$

denote the associated projection map. 
Lemma 3.4. Let $E, \Xi, \Lambda$ be as above. Then for each $\Psi \in \operatorname{End}\left(V_{E, \Xi, \Lambda}\right)_{K \times K}^{*}$ there exists a finite sequence $\left(\xi_{i}, \psi_{i}, \lambda_{i}, u_{i}\right)$ in $\Sigma$ such that for all $\varphi \in \mathcal{O}\left(\mathfrak{a}_{\mathbb{C}}^{*}\right) \otimes$ $\mathcal{S}\left(P_{0}\right)$

$$
\left\langle\varphi_{E, \Xi, \Lambda}, \Psi\right\rangle=\sum_{i}\left\langle\varphi_{\xi_{i}, \lambda_{i} ; u_{i}}, \psi_{i}\right\rangle
$$

Proof. Put

$$
\mathcal{S}\left(P_{0}, \Xi, \Lambda\right):=\oplus_{(\xi, \lambda) \in \Xi \times \Lambda} \mathcal{S}\left(P_{0}, \xi\right) .
$$

We observe that $\operatorname{End}\left(V_{E, \Xi, \Lambda}\right)_{K \times K}^{*}$ may be viewed as the direct sum of the $K \times K$-submodule $\operatorname{End}(E)^{*} \otimes \mathcal{S}\left(P_{0}, \Xi, \Lambda\right)_{K \times K}^{*}$ and a unique $K \times K$ submodule $\operatorname{End}(E)^{*} \otimes \mathcal{T}$ (consisting of the 'cross terms'). Since every element of $\operatorname{End}(E)^{*} \otimes \mathcal{T}$ annihilates $\varphi_{E, \Xi, \Lambda}$, we may assume that $\Psi \in$ $\operatorname{End}(E)^{*} \otimes \mathcal{S}\left(P_{0}, \Xi, \Lambda\right)_{K \times K}^{*}$. By linearity, we may then reduce to the situation that $\Xi=\{\xi\}$ and $\Lambda=\{\lambda\}$. Again by linearity we may assume that $\Psi$ is of the form $\eta \otimes \psi$, with $\eta \in \operatorname{End}(E)^{*}$ and $\psi \in \mathcal{S}\left(P_{0}, \xi\right)_{K \times K}^{*}$. Let $u \in S\left(\mathfrak{a}^{*}\right)$ be associated with $\eta$ as in Lemma 2.10, Then for all $\varphi \in \mathcal{O}\left(\mathfrak{a}_{\mathbb{C}}^{*}\right) \otimes \mathcal{S}\left(P_{0}\right)$,

$$
\left\langle\varphi_{E, \xi, \lambda}, \Psi\right\rangle=\left\langle\varphi_{\xi, \lambda}^{(E)}, \eta \otimes \psi\right\rangle=\left\langle\varphi_{\xi, \lambda ; u}, \psi\right\rangle .
$$

This finishes the proof.

Lemma 3.5. Let $\left(\xi_{i}, \psi_{i}, \lambda_{i}, u_{i}\right)$ be a finite sequence in $\Sigma$. Then there exists a finite dimensional $\mathcal{O}_{0}$-module $E$, and finite sets $\Xi \subset M^{\wedge}, \Lambda \subset \mathfrak{a}_{\mathbb{C}}^{*}$ and an element $\Psi \in \operatorname{End}\left(V_{E, \Xi, \Lambda}\right)_{K \times K}^{*}$ such that for all $\varphi \in \mathcal{O}\left(\mathfrak{a}_{\mathbb{C}}^{*}\right) \otimes \mathcal{S}\left(P_{0}\right)$,

$$
\sum_{i}\left\langle\varphi_{\xi_{i}, \lambda_{i} ; u_{i}}, \psi_{i}\right\rangle=\left\langle\varphi_{E, \Xi, \Lambda}, \Psi\right\rangle .
$$

Proof. Let $E$ and $\eta_{i} \in \operatorname{End}(E)^{*}$ be associated to the finite sequence $u_{i}$ as in Lemma 2.11. Then $\Psi_{i}=\eta_{i} \otimes \psi_{i}$ belongs to $\operatorname{End}(E)^{*} \otimes \mathcal{S}\left(P_{0}, \xi_{i}\right)_{K \times K}^{*} \simeq$ $\operatorname{End}\left(V_{E, \xi_{i}, \lambda_{i}}\right)_{K \times K}^{*}$. Let $\Xi$ be the finite set of all $\xi_{i}$ and let $\Lambda$ be the finite set of all $\lambda_{i}$. Let $\operatorname{pr}_{\xi_{i}, \lambda_{i}}$ be defined as above. We define $\Psi \in \operatorname{End}\left(V_{E, \Xi, \Lambda}\right)_{K \times K}^{*}$ by

$$
\Psi=\sum_{i} \operatorname{pr}_{\xi_{i}, \lambda_{i}}^{*}\left(\eta_{i} \otimes \psi_{i}\right)
$$

Then for all $\varphi \in \mathcal{O}\left(\mathfrak{a}_{\mathbb{C}}^{*}\right) \otimes \mathcal{S}\left(P_{0}\right)$ we have

$$
\begin{aligned}
\sum_{i}\left\langle\varphi_{\xi_{i}, \lambda_{i} ; u_{i}}, \psi_{i}\right\rangle & =\sum_{i}\left\langle\varphi_{\xi_{i}, \lambda_{i}}^{(E)}, \eta_{i} \otimes \psi_{i}\right\rangle \\
& =\sum_{i}\left\langle\operatorname{pr}_{\xi_{i}, \lambda_{i}} \varphi_{E, \Xi, \Lambda}, \eta_{i} \otimes \psi_{i}\right\rangle \\
& =\left\langle\varphi_{E, \Xi, \Lambda}, \Psi\right\rangle .
\end{aligned}
$$

Proof of Proposition 3.3. Let $\phi$ be as stated and assume (a). Let $E, \Xi, \Lambda$ and $\Psi$ be as asserted in (b). In particular, $\Psi \in \operatorname{End}\left(V_{E, \Xi, \Lambda}\right)_{K \times K}^{*}$. Let $\left(\xi_{i}, \psi_{i}, \lambda_{i}, u_{i}\right)$ be a sequence in $\Sigma$, associated to $\Psi$ as in Lemma 3.4. Then using the relation (3.8) with $\varphi=\hat{f}\left(P_{0}\right)$ for $f \in C_{c}^{\infty}(G, K)$, we see that $\left(\xi_{i}, \psi_{i}, \lambda_{i}, u_{i}\right)$ is an Arthur-Campoli sequence (see (3.4)). Hence,

$$
\left\langle\phi_{E, \Xi, \Lambda}, \Psi\right\rangle=\sum_{i}\left\langle\phi_{\xi_{i}, \lambda_{i} ; u_{i}}, \psi_{i}\right\rangle=0 .
$$


We have proved (b).

Conversely, assume (b) and let $\left(\xi_{i}, \psi_{i}, \lambda_{i}, u_{i}\right)$ be an Arthur-Campoli sequence in $\Sigma$. Let $E, \Xi, \Lambda, \Psi$ be associated with this sequence as in Lemma 3.5. Then it follows from (3.9) with $\varphi=\hat{f}\left(P_{0}\right)$ for $f \in C_{c}^{\infty}(G, K)$ that $\Psi$ belongs to $\pi_{E, \Xi, \Lambda}\left(C_{c}^{\infty}(G, K)\right)^{\perp}$. This implies that

$$
\sum_{i}\left\langle\phi_{\xi_{i}, \lambda_{i} ; u_{i}}, \psi_{i}\right\rangle=\left\langle\phi_{E, \Xi, \Lambda}, \Psi\right\rangle=0 .
$$

Hence (a).

\section{Delorme's interWining CONDitions}

4.1. Successive derivatives. Let $\mathfrak{v}$ be a finite dimensional vector space over $\mathbb{R}$, let $\Omega \subset \mathfrak{v}_{\mathbb{C}}^{*}$ be an open subset and let $V$ be a quasi-complete locally convex space. Following Delorme [5], we define, for $\Phi \in \mathcal{O}(\Omega$, End $(V))$ and $\eta \in \mathfrak{v}_{\mathbb{C}}^{*}$, the holomorphic function $\Phi^{(\eta)}: \Omega \rightarrow \operatorname{End}(V \oplus V)$ by

$$
\Phi^{(\eta)}(\lambda)\left(v_{1}, v_{2}\right):=\left(\Phi(\lambda) v_{1}+\frac{d}{d z}\left(\Phi(\lambda+z \eta) v_{2}\right)_{\mid z=0}, \Phi(\lambda) v_{2}\right),
$$

for $\lambda \in \Omega$ and $v_{1}, v_{2} \in V$. Still following [5], we define, for any finite sequence $\eta=\left(\eta_{1}, \ldots, \eta_{N}\right)$ in $\mathfrak{v}_{\mathbb{C}}^{*}$, the iterated derivative

$$
\Phi^{(\eta)}:=\left(\cdots\left(\Phi^{\left(\eta_{N}\right)}\right)^{\left(\eta_{N-1}\right)} \cdots\right)^{\left(\eta_{1}\right)}
$$

of $\Phi$ along $\eta$. Then $\Phi^{(\eta)}$ is a holomorphic function on $\Omega$ with values in $\operatorname{End}\left(V^{(\eta)}\right)$, where $V^{(\eta)}$ denotes the direct sum of $2^{N}$ copies of $V$.

Now assume that $V$ is Fréchet (or more generally, barrelled). If $\pi$ is a holomorphic family of continuous representations of $G$ in $V$ over the parameter set $\mathfrak{v}_{\mathbb{C}}^{*}$ then it follows by application of the methods of [5] that for each $\lambda \in \Omega$,

$$
\pi_{\lambda}^{(\eta)}(x):=\pi(x)^{(\eta)}(\lambda), \quad x \in G,
$$

defines a continuous representation of $G$ in $V^{(\eta)}$.

4.2. The intertwining conditions. Let $\mathcal{D}$ be the set of 4-tuples $\delta=$ $(P, \xi, \lambda, \eta)$, with $P \in \mathcal{P}(A), \xi \in M_{P, d s}^{\wedge}, \lambda \in \mathfrak{a}_{P \mathbb{C}}^{*}$ and $\eta$ a finite sequence in $\mathfrak{a}_{P \mathbb{C}}^{*}$. Given $\delta \in \mathcal{D}$, we define the representation $\pi_{\delta}$ of $G$ in $V_{\pi_{\delta}}:=C^{\infty}(K: \xi)^{(\eta)}$ by

$$
\pi_{\delta}:=\pi_{P, \xi, \lambda}^{(\eta)}
$$

For $P \in \mathcal{P}(A)$ we define the space $\mathcal{F}_{P}:=\mathcal{O}\left(\mathfrak{a}_{P \mathbb{C}}^{*}\right) \otimes \mathcal{S}(P)$, with $\mathcal{S}(P)$ defined as in (3.2). Furthermore, we put

$$
\mathcal{F}:=\oplus_{P \in \mathcal{P}(A)} \mathcal{F}_{P}
$$

Given $\varphi \in \mathcal{F}$ and $\delta=(P, \xi, \lambda, \eta) \in \mathcal{D}$, we define $\varphi_{\delta} \in \operatorname{End}\left(C^{\infty}(K: \xi)^{(\eta)}\right)$ in a similar fashion as $\pi_{\delta}$, by

$$
\varphi_{\delta}:=\varphi_{P, \xi}^{(\eta)}(\lambda) .
$$

Finally, given a sequence $\delta=\left(\delta_{1}, \ldots, \delta_{N}\right)$ of data from $\mathcal{D}$, we define $\pi_{\delta}:=\pi_{\delta_{1}} \oplus \cdots \oplus \pi_{\delta_{N}}, V_{\pi_{\delta}}:=V_{\pi_{\delta_{1}}} \oplus \cdots \oplus V_{\pi_{\delta_{N}}}$ and $\varphi_{\delta}:=\varphi_{\delta_{1}} \oplus \cdots \oplus \varphi_{\delta_{N}}$. 
Definition 4.1. We say that a function $\varphi \in \mathcal{F}$ satisfies Delorme's intertwining conditions (see [5, Definition $3(4.4)]$ ) if

(a) for every $N \in \mathbb{Z}_{+}$and each $\delta \in \mathcal{D}^{N}$ the function $\varphi_{\delta}$ preserves all invariant subspaces of $\pi_{\delta}$;

(b) for all $N_{1}, N_{2} \in \mathbb{Z}_{+}$, all $\delta_{1} \in \mathcal{D}^{N_{1}}$ and $\delta_{2} \in \mathcal{D}^{N_{2}}$, and any two sequences of closed invariant subspaces $U_{j} \subset V_{j}$ for $\pi_{\delta_{j}}$, the induced maps $\bar{\varphi}_{\delta_{j}} \in \operatorname{End}\left(V_{j} / U_{j}\right)$ are intertwined by all intertwining operators $T: V_{1} / U_{1} \rightarrow V_{2} / U_{2}$.

The space of functions $\varphi \in \mathcal{F}$ satisfying (a) and (b) is denoted by $\mathcal{F}(\mathcal{D})$.

4.3. A simplification of the intertwining conditions. In this section we will show that condition (b) of Definition 4.1 is in fact a consequence of condition (a) of the same definition.

Lemma 4.2. Let $\varphi \in \mathcal{F}$. Then $\varphi \in \mathcal{F}(\mathcal{D})$ if and only if $\varphi$ satisfies condition (a) of Definition 4.1.

Proof. Assume that $\varphi$ satisfies condition (a) of the mentioned definition. Then we must show that $\varphi$ satisfies condition (b) as well.

Let $\delta_{j}, \pi_{\delta_{j}}, U_{j}, V_{j}$ be as in (b), for $j=1,2$. Let $T: V_{1} / U_{1} \rightarrow V_{2} / U_{2}$ be an intertwining operator. Let $p_{j}: V_{j} \rightarrow V_{j} / U_{j}$ denote the canonical projection, for $j=1,2$, and let $p:=p_{1} \oplus p_{2}$. Since $T$ is equivariant, its graph $W$ is an invariant subspace of $V_{1} / U_{1} \oplus V_{2} / U_{2}$. Hence, $p^{-1}(W)$ is an invariant subspace of $V_{1} \oplus V_{2}$. Since $\varphi$ satisfies (a), it follows that $p^{-1}(W)$ is a $\varphi_{\delta_{1}} \oplus \varphi_{\delta_{2}}$ invariant subspace of $V_{1} \oplus V_{2}$. This in turn is easily seen to imply that $T \circ \bar{\varphi}_{\delta_{1}}=\bar{\varphi}_{\delta_{2}} \circ T$.

4.4. Reduction to a single minimal parabolic subgroup. In this section, we will show that the space $\mathcal{F}(\mathcal{D})$ of functions satisfying Delorme's intertwining conditions is naturally isomorphic to a space of functions defined in terms of just the minimal parabolic subgroup $P_{0}$. We denote by $\mathcal{D}_{P_{0}}$ the set of 4 -tuples $(P, \xi, \lambda, \eta)$ in $\mathcal{D}$ for which $P=P_{0}$.

Definition 4.3. We say that a function $\varphi \in \mathcal{F}$ satisfies Delorme's intertwining conditions associated with $P_{0}$ if conditions (a) and (b) of Definition 4.1 are valid with everywhere $\mathcal{D}$ replaced by $\mathcal{D}_{P_{0}}$.

The space of functions $\varphi \in \mathcal{F}$ satisfying all such intertwining conditions is denoted by $\mathcal{F}\left(\mathcal{D}_{P_{0}}\right)$.

The following analogue of Lemma 4.2 is now valid, with essentially the same proof.

Lemma 4.4. Let $\varphi \in \mathcal{F}$. Then $\varphi \in \mathcal{F}\left(\mathcal{D}_{P_{0}}\right)$ if and only if $\varphi$ satisfies condition (a) of Definition 4.1 for every $N \in \mathbb{Z}_{+}$and all $\delta \in\left(\mathcal{D}_{P_{0}}\right)^{N}$.

We consider the component

$$
\mathcal{F}_{P_{0}}:=\mathcal{O}\left(\mathfrak{a}_{\mathbb{C}}^{*}\right) \otimes \mathcal{S}\left(P_{0}\right)
$$

of the direct sum $\mathcal{F}$ defined in (4.1), and denote the natural projection $\mathcal{F} \rightarrow \mathcal{F}_{P_{0}}$ by pr. Moreover, we define

$$
\begin{aligned}
& \mathcal{F}_{P_{0}}\left(\mathcal{D}_{P_{0}}\right):= \mathcal{F}_{P_{0}} \cap \mathcal{F}\left(\mathcal{D}_{P_{0}}\right) . \\
& 33
\end{aligned}
$$


Proposition 4.5. The natural projection pr: $\mathcal{F} \rightarrow \mathcal{F}_{P_{0}}$ restricts to a linear isomorphism from $\mathcal{F}(\mathcal{D})$ onto $\mathcal{F}_{P_{0}}\left(\mathcal{D}_{P_{0}}\right)$.

Proof. If $\varphi \in \mathcal{F}$ and if $\delta$ is a finite sequence in $\mathcal{D}_{P_{0}}$, then $\varphi_{\delta}=\left(\varphi_{P_{0}}\right)_{\delta}$. Hence $\varphi \in \mathcal{F}\left(\mathcal{D}_{P_{0}}\right)$ if and only if $\operatorname{pr}(\varphi) \in \mathcal{F}\left(\mathcal{D}_{P_{0}}\right)$.

From $\mathcal{D}_{P_{0}} \subset \mathcal{D}$ it follows that $\mathcal{F}(\mathcal{D}) \subset \mathcal{F}\left(\mathcal{D}_{0}\right)$. Therefore, pr maps $\mathcal{F}(\mathcal{D})$ into $\mathcal{F}_{P_{0}} \cap \mathcal{F}\left(\mathcal{D}_{P_{0}}\right)=\mathcal{F}_{P_{0}}\left(\mathcal{D}_{P_{0}}\right)$. The proof will be completed by showing that the restricted projection $\operatorname{pr}_{\mathcal{D}}:=\left.\operatorname{pr}\right|_{\mathcal{F}(\mathcal{D})}: \mathcal{F}(\mathcal{D}) \rightarrow \mathcal{F}_{P_{0}}\left(\mathcal{D}_{P_{0}}\right)$ is a linear isomorphism. We will do this by defining a map

$$
\tau: \mathcal{F}_{P_{0}} \longrightarrow \mathcal{F}
$$

which will turn out to induce a two-sided inverse for $\mathrm{pr}_{\mathcal{D}}$.

Each parabolic subgroup $P \in \mathcal{P}(A)$ has a Langlands decomposition $P=$ $M_{P} A_{P} N_{P}$. Here $M_{P}$ is a real reductive group of the Harish-Chandra class, with Cartan decomposition $M_{P}=\left(M_{P} \cap K\right) \exp \left(\mathfrak{m}_{P} \cap \mathfrak{p}\right)$. Moreover, ${ }^{*} \mathfrak{a}:=$ $\mathfrak{m}_{P} \cap \mathfrak{a}$ is maximal abelian in $\mathfrak{m}_{P} \cap \mathfrak{p}$, and the centralizer of ${ }^{*} \mathfrak{a}$ in $K_{P}:=$ $M_{P} \cap K$ equals $M=Z_{K}(\mathfrak{a})$. We select a minimal parabolic subgroup

$$
{ }^{*} Q=M^{*} A^{*} N
$$

of $M_{P}$. In addition, we fix a minimal parabolic subgroup $Q$ of $G$ containing $A$ such that ${ }^{*} Q=Q \cap M_{P}$, and we fix an element $w \in N_{K}(\mathfrak{a})$ such that $P_{0}=w^{-1} Q w$. In case $P=P_{0}$, we agree to make the special choice $Q=P_{0}$ and $w=e$.

Then by the subrepresentation theorem applied to the group $M_{P}$, for each $\xi \in M_{P, d s}^{\wedge}$ we may fix a representation $\sigma \in M^{\wedge}$ and an element $\mu \in$ ${ }^{*} \mathfrak{a}_{\mathbb{C}}^{*}$ such that $\xi$ is equivalent to a subrepresentation of the parabolically induced (smooth) representation $\pi_{* Q, \sigma, \mu}^{M_{P}}$ of $M_{P}$. In addition, we fix an $M_{P^{-}}$ equivariant embedding

$$
j_{\xi}:\left(\xi, V_{\xi}\right) \hookrightarrow\left(\pi_{* Q, \sigma, \mu}^{M_{P}}, C^{\infty}\left(K \cap M_{P}: \sigma\right)\right) .
$$

Through induction by stages, this embedding induces an embedding

$$
j_{\xi P}^{G}: C^{\infty}(K: \xi) \longrightarrow C^{\infty}(K: \sigma)
$$

which intertwines $\pi_{P, \xi, \lambda}$ with $\pi_{Q, \sigma, \mu+\lambda}$, for all $\lambda \in \mathfrak{a}_{P \mathbb{C}}^{*}$. Here ${ }^{*} \mathfrak{a}_{\mathbb{C}}^{*}$ and $\mathfrak{a}_{P \mathbb{C}}^{*}$ are viewed as subspaces of $\mathfrak{a}_{\mathbb{C}}^{*}$ via the direct sum decomposition $\mathfrak{a}={ }^{*} \mathfrak{a} \oplus \mathfrak{a}_{P}$. Thus, (4.2) is a morphism in the category ${ }^{\circ} \mathrm{HF}_{G}$, see Definition 2.38. In the special case $P=P_{0}$, we agreed that $Q=P_{0}$ and $w=e$. In this case, we have $\mu=0$, and as $\sigma$ must be equivalent to $\xi$, it follows that $\sigma=w \cdot \sigma=\xi$. It is now readily verified that $j_{\xi P}^{G}$ is the identity map of $C^{\infty}(K: \xi)$.

Left translation by $w$ induces a topological linear isomorphism

$$
L(w): C^{\infty}(K: \sigma) \longrightarrow C^{\infty}(K: w \sigma),
$$

which intertwines the induced representation $\pi_{Q, \sigma, \mu+\lambda}$ with $\pi_{P_{0}, w \sigma, w(\mu+\lambda)}$, for all $\lambda \in \mathfrak{a}_{P \mathbb{C}}^{*}$. Here $w \sigma$ denotes the representation of $M$ in $V_{\sigma}$, given by $w \sigma(m)=\sigma\left(w^{-1} m w\right)$. We denote by $\left(w \cdot \sigma, V_{w \cdot \sigma}\right)$ the unique element of $M^{\wedge}$ which is equivalent to $\left(w \sigma, V_{\sigma}\right)$. Fix an equivalence $t_{w}: V_{\sigma} \rightarrow V_{w \cdot \sigma}$ and denote the induced map $C^{\infty}(K: w \sigma) \rightarrow C^{\infty}(K: w \cdot \sigma)$ by $T_{w}$. Then writing $\mathcal{L}_{w}=T_{w} \circ L(w)$, we obtain a continuous linear injection

$$
\mathcal{L}_{w} \circ j_{\xi P}^{G}: C^{\infty}(K: \xi) \longrightarrow C^{\infty}(K: w \cdot \sigma)
$$


which intertwines the representation $\pi_{P, \xi, \lambda}$ with $\pi_{P_{0}, w \cdot \sigma, w(\mu+\lambda)}$, for all $\lambda \in$ $\mathfrak{a}_{P \mathbb{C}}^{*}$. In the special case $P=P_{0}$, where $Q=P_{0}, w=e, \mu=0$ and $\sigma=\xi$, we may take $t_{w}=\operatorname{id}_{V_{\xi}}$ and then $\mathcal{L}_{w}$ and hence (4.4) become the identity map of $C^{\infty}(K: \xi)$.

We are now ready to define the map $\tau$. Let $P, \xi, \lambda$ be as above, and let $\varphi \in \mathcal{F}_{P_{0}}\left(\mathcal{D}_{0}\right)$. Then $\varphi_{P_{0}, w \cdot \sigma, w(\mu+\lambda)}$ leaves the invariant subspace $\operatorname{im}\left(\mathcal{L}_{w} \circ j_{\xi P}^{G}\right)$ for the representation $\pi_{P_{0}, w \cdot \sigma, w(\mu+\lambda)}$ invariant by condition (a) of Definition 4.1, so that we may define

$$
\tau(\varphi)_{P, \xi, \lambda}: C^{\infty}(K: \xi) \rightarrow C^{\infty}(K: \xi)
$$

to be the unique linear map such that the following diagram commutes

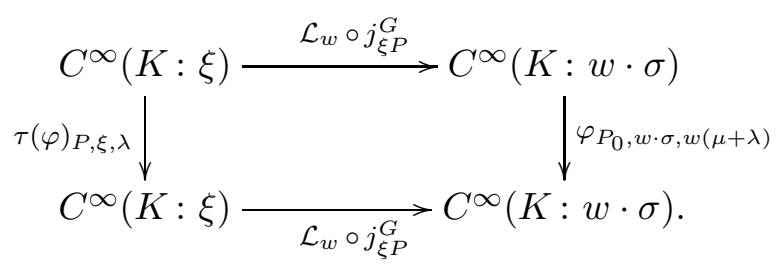

Since $\varphi_{P_{0}, w \cdot \sigma} \in \mathcal{O}\left(\mathfrak{a}_{\mathbb{C}}^{*}\right) \otimes \operatorname{End}\left(C^{\infty}(K: w \cdot \sigma)\right)_{K \times K}$, it is readily seen that $\tau(\varphi)_{P, \xi}$ defines a holomorphic function on $\mathfrak{a}_{P \mathbb{C}}^{*}$, with values in $\mathcal{S}(P, \xi)$. Accordingly, $\tau$ defines a linear map $\mathcal{F}_{P_{0}} \rightarrow \mathcal{F}$.

We will now finish the proof by showing that

(i) $\tau$ maps $\mathcal{F}_{P_{0}}\left(\mathcal{D}_{P_{0}}\right)$ into $\mathcal{F}(\mathcal{D})$,

(ii) $\tau$ restricts to a linear isomorphism $\tau_{\mathcal{D}}: \mathcal{F}_{P_{0}}\left(\mathcal{D}_{P_{0}}\right) \rightarrow \mathcal{F}(\mathcal{D})$ which is a two-sided inverse for $\mathrm{pr}_{\mathcal{D}}$.

We will first establish (i). Let $\varphi \in \mathcal{F}_{P_{0}}\left(\mathcal{D}_{0}\right)$, and let $N \in \mathbb{Z}_{+}$and $\delta \in \mathcal{D}^{N}$. We claim that it suffices to show that there exists a $\delta^{\prime} \in \mathcal{D}_{P_{0}}^{N}$ and a linear embedding $j: V_{\delta} \rightarrow V_{\delta^{\prime}}$ intertwining $\pi_{\delta}$ with $\pi_{\delta^{\prime}}$ such that

$$
\varphi_{\delta^{\prime}} \circ j=j \circ \tau(\varphi)_{\delta} .
$$

Indeed assume the claim to hold, and let $W \subset V_{\delta}$ be an invariant subspace. Then $j(W)$ is an invariant subspace of $V_{\delta^{\prime}}$. Moreover, since $\varphi \in \mathcal{F}_{P_{0}}\left(\mathcal{D}_{0}\right)$, it follows that $\varphi_{\delta^{\prime}}$ leaves $j(W)$ invariant. As $j$ is injective, it now follows from (4.6) that $\tau(\varphi)_{\delta}$ leaves $W$ invariant. Thus, the validity of the claim would imply that the above condition (i) holds.

We turn to the proof of the claim. It clearly suffices to prove the claim in case $N=1$, so that $\delta \in \mathcal{D}$. Thus, $\delta$ is a 4 -tuple of the form $\left(P, \xi, \lambda_{0}, \eta\right)$ with notation as in the beginning of Section 4.2. In particular, $\eta$ is a finite sequence in $\mathfrak{a}_{P \mathbb{C}}^{*}$. Let $Q, \sigma, \mu, w$ be associated with the data $P, \xi$ as in the first part of this proof, where the definition of $\tau$ was given. Then the injective linear map (4.4) intertwines $\pi_{P, \xi, \lambda}$ with $\pi_{P_{0}, w \cdot \sigma, w(\mu+\lambda)}$ for all $\lambda \in \mathfrak{a}_{P \mathbb{C}}^{*}$.

It follows that the map

$$
j: C^{\infty}(K: \xi)^{(\eta)} \longrightarrow C^{\infty}(K: w \cdot \sigma)^{(\eta)}
$$

induced by (4.4) interwines $\pi_{\delta}$ with $\pi_{\delta^{\prime}}$, where $\delta^{\prime}=\left(P_{0}, w \cdot \sigma, w\left(\lambda_{0}+\mu\right), w \eta\right)$. Moreover, the commutativity of the diagram (4.5) implies that

$$
j \circ \tau(\varphi)_{P, \xi, \lambda}^{(\eta)}=\varphi_{P_{0}, w \cdot \sigma, w(\mu+\lambda)}^{(w \eta)} \circ j,
$$

or, abbreviated, $j \circ \tau(\varphi)_{\delta}=\varphi_{\delta^{\prime}} \circ j$. This establishes the claim. 
We now consider the induced map $\tau_{\mathcal{D}}: \mathcal{F}_{P_{0}}\left(\mathcal{D}_{0}\right) \rightarrow \mathcal{F}(\mathcal{D})$, obtained by restriction of $\tau$, and will finish the proof by establishing (ii).

Let $\varphi \in \mathcal{F}_{P_{0}}\left(\mathcal{D}_{P_{0}}\right)$. Moreover, let $Q, \sigma, \mu, w$ be associated to $P=P_{0}$ as above. By the special choices we made for this particular parabolic subgroup, the diagram (4.5) becomes

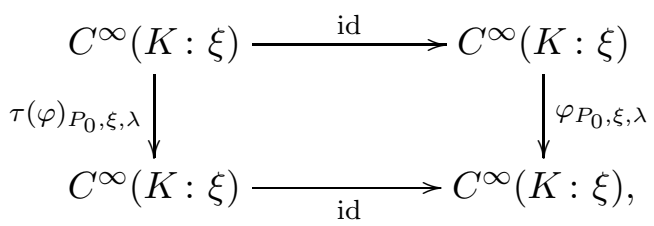

It follows that $\operatorname{pr} \circ \tau(\varphi)=\varphi$, and we see that $\tau_{\mathcal{D}}$ is a right inverse to $\operatorname{pr}_{\mathcal{D}}$.

We will finish the proof by showing that $\tau_{\mathcal{D}}$ is also a left inverse. Let $\psi \in \mathcal{F}(\mathcal{D})$, and let $P \in \mathcal{P}(A)$ and $\xi \in M_{P, d s}^{\wedge}$. Let $Q, \sigma, \mu, w$ be as above. As $\psi$ satisfies condition (b) of Definition 4.1, it follows that the following diagram commutes, for all $\lambda \in \mathfrak{a}_{P \mathbb{C}}^{*}$,

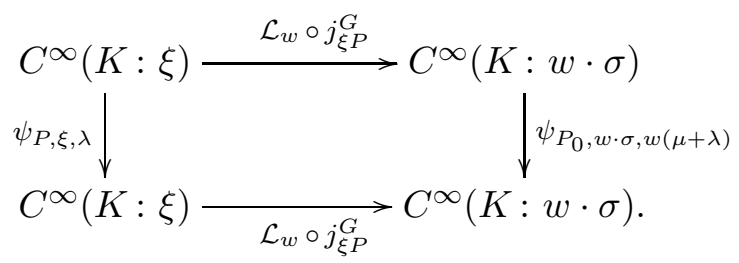

By comparison with (4.5) we see that $\psi_{P, \xi}=\tau\left(\psi_{P_{0}}\right)_{P, \xi}$. Hence, $\tau \circ \operatorname{pr}(\psi)=\psi$, for $\psi \in \mathcal{F}(\mathcal{D})$. Therefore, $\tau_{\mathcal{D}}$ is a left inverse to $\operatorname{pr}_{\mathcal{D}}$.

4.5. Reformulation in our setting. We shall now compare Delorme's derivation process with the process defined in the present paper. In the following we shall identify $V \oplus V$ with $\mathbb{C}^{2} \otimes V$ via the map $\left(v_{1}, v_{2}\right) \mapsto$ $(1,0) \otimes v_{1}+(0,1) \otimes v_{2}$. This identification induces an identification $\operatorname{End}(V \oplus$ $V) \simeq \operatorname{End}\left(\mathbb{C}^{2}\right) \otimes \operatorname{End}(V)$.

Let now $\eta \in \mathfrak{a}_{\mathbb{C}}^{*}$ and let $\Omega$ be an open subset of $\mathfrak{a}_{\mathbb{C}}^{*}$. For $\varphi \in \mathcal{O}(\Omega)$ we define $D^{(\eta)} \varphi \in \mathcal{O}(\Omega) \otimes \operatorname{End}\left(\mathbb{C}^{2}\right)$ by

$$
D^{(\eta)} \varphi(\lambda)=\left(\begin{array}{cc}
\varphi(\lambda) & \varphi(\lambda ; \eta) \\
0 & \varphi(\lambda)
\end{array}\right) .
$$

Let $V$ be a quasi-complete locally convex space. Then for $\Phi \in \mathcal{O}(\Omega) \otimes$ $\operatorname{End}(V)$, Delorme's derivative $\Phi^{(\eta)}$ is given by

$$
\Phi^{(\eta)}=\left(D^{(\eta)} \otimes \operatorname{id}_{\operatorname{End}(V)}\right) \Phi .
$$

Here we note that

$$
V^{(\eta)}=\mathbb{C}^{2} \otimes V,
$$

so that $\operatorname{End}\left(V^{(\eta)}\right)=\operatorname{End}\left(\mathbb{C}^{2}\right) \otimes \operatorname{End}(V)$. It follows that Delorme's differentiation map $(\cdot)^{(\eta)}$ from $\mathcal{O}(\Omega, \operatorname{End}(V))$ to $\mathcal{O}\left(\Omega, \operatorname{End}\left(V^{(\eta)}\right)\right)$ is the unique continuous linear extension of the map $D^{(\eta)} \otimes \operatorname{id}_{\operatorname{End}(V)}$.

Now assume that $\eta \neq 0$ and let $\mathcal{I}_{\eta}$ be the cofinite ideal of $\mathcal{O}_{0}=\mathcal{O}_{0}\left(\mathfrak{a}_{\mathbb{C}}^{*}\right)$ defined in (2.14). Then $\mathcal{I}_{\eta}$ has codimension 2 by Lemma 2.27. More precisely, 
let $X \in \mathfrak{a}_{\mathbb{C}}$ be such that $\eta(X)=1$. Then the map $\left(z_{1}, z_{2}\right) \mapsto z_{1}+z_{2} X+\mathcal{I}_{\eta}$ defines a linear isomorphism $\kappa$ from $\mathbb{C}^{2}$ onto the $\mathcal{O}_{0}$-module

$$
E_{\eta}:=\mathcal{O}_{0} / \mathcal{I}_{\eta} \text {. }
$$

Now assume that $V$ is barrelled. Then in view of (4.7) the isomorphism $\kappa$ induces an isomorphism from $\mathcal{O}\left(\Omega, \operatorname{End}\left(V^{(\eta)}\right)\right)$ onto $\mathcal{O}\left(\Omega, \operatorname{End}\left(E_{\eta} \otimes V\right)\right)$, denoted $\kappa_{*}$.

Lemma 4.6. With notation as above, the following diagram commutes

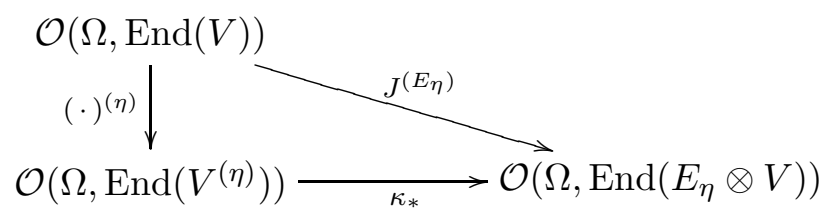

Proof. By the same calculation as in the proof of Lemma 2.28, it follows that the multiplication action $m(\varphi)$ of an element $\varphi \in \mathcal{O}_{0}$ on $E_{\eta}$ with respect to the basis $\overline{1}, \bar{X}$ is given by the matrix

$$
M(\varphi):=\left(\begin{array}{cc}
\varphi(0) & \varphi(0 ; \eta) \\
0 & \varphi(0)
\end{array}\right)
$$

Let $\widetilde{\kappa}$ be the isomorphism $\operatorname{End}\left(\mathbb{C}^{2}\right) \rightarrow \operatorname{End}\left(E_{\eta}\right)$ induced by $\kappa$. Then it follows that for all $\varphi \in \mathcal{O}(\Omega)$ and $\lambda \in \Omega$,

$$
J^{\left(E_{\eta}\right)} \varphi(\lambda)=m\left(\gamma_{0}\left(T_{\lambda} \varphi\right)\right)=\widetilde{\kappa}\left(M\left(\gamma_{0}\left(T_{\lambda} \varphi\right)\right)\right)=\widetilde{\kappa}\left(D^{(\eta)} \varphi(\lambda)\right) .
$$

This immediately implies the commutativity of the diagram in case $V=\mathbb{C}$. From this we see that the diagram commutes with the spaces $\mathcal{O}(\Omega, \operatorname{End}(W))$ replaced by the subspaces $\mathcal{O}(\Omega) \otimes \operatorname{End}(W)$, for $W$ equal to $V, V^{(\eta)}$ or $E_{\eta} \otimes V$. By density of the mentioned spaces and continuity of the maps involved, the result follows.

Given a finite sequence $\eta=\left(\eta_{1}, \ldots, \eta_{N}\right)$ of elements in $\mathfrak{a}_{\mathbb{C}}^{*}$, we define the $\mathcal{O}_{0}$-module $E_{\eta}:=E_{\eta_{1}} \otimes \cdots \otimes E_{\eta_{N}}$.

Corollary 4.7. For every finite sequence $\eta$ as above, and all $\xi \in M^{\wedge}$ and $\lambda \in \mathfrak{a}_{\mathbb{C}}^{*}$,

$$
\pi_{P_{0}, \xi, \lambda}^{(\eta)} \simeq \pi_{P_{0}, \xi, \lambda}^{\left(E_{\eta}\right)} .
$$

Proof. For $\eta$ of length one this follows from the above lemma. For arbitrary sequences it follows from the recurrent nature of the definition of $\pi^{(\eta)}$ and Proposition 2.26.

Definition 4.8. Let $V$ be a Harish-Chandra module. We denote by $\operatorname{End}(V)^{\#}$ the space of endomorphisms $\varphi \in \operatorname{End}(V)_{K \times K}$ such that for every $n \in \mathbb{N}$ the product map $\varphi^{\times n} \in \operatorname{End}\left(V^{\times n}\right)$ leaves all $(\mathfrak{g}, K)$-invariant subspaces of $V^{\times n}$ invariant.

If $\left(\pi, V_{\pi}\right)$ is an admissible representation of $G$ of finite length, we define $\operatorname{End}(\pi)^{\#}:=\operatorname{End}\left(\left(V_{\pi}\right)_{K}\right)^{\#}$.

For $E$ a finite dimensional $\mathcal{O}_{0}$-module (where $\mathcal{O}_{0}=\mathcal{O}_{0}\left(\mathfrak{a}_{\mathbb{C}}^{*}\right)$ ), and for $\Xi \subset$ $M^{\wedge}$ and $\Lambda \subset \mathfrak{a}_{\mathbb{C}}^{*}$ finite subsets, we recall the definition of the representation $\pi_{E, \Xi, \Lambda}$ by (3.5). Moreover, given $\varphi \in \mathcal{F}_{P_{0}}=\mathcal{O}\left(\mathfrak{a}_{\mathbb{C}}^{*}\right) \otimes \mathcal{S}\left(P_{0}\right)$, we recall the definition of $\varphi_{E, \Xi, \Lambda}$ in (3.7). 
Lemma 4.9. Let $E, E^{\prime}$ be finite dimensional $\mathcal{O}_{0}$-modules such that $E$ is a subquotient of $E^{\prime}$. Let $\Xi \subset \Xi^{\prime} \subset M^{\wedge}$ be finite subsets and let $\Lambda \subset \Lambda^{\prime} \subset \mathfrak{a}_{\mathbb{C}}^{*}$ be finite subsets. Then for all $\varphi \in \mathcal{F}_{P_{0}}$,

$$
\varphi_{E^{\prime}, \Xi^{\prime}, \Lambda^{\prime}} \in \operatorname{End}\left(\pi_{E^{\prime}, \Xi^{\prime}, \Lambda^{\prime}}\right)^{\#} \Longrightarrow \varphi_{E, \Xi, \Lambda} \in \operatorname{End}\left(\pi_{E, \Xi, \Lambda}\right)^{\#}
$$

Proof. The result follows from the crucial observation that the representation $\pi_{E, \Xi, \Lambda}$ is a subquotient of $\pi_{E^{\prime}, \Xi^{\prime}, \Lambda^{\prime}}$ and the map $\varphi_{E^{\prime}, \Xi^{\prime}, \Lambda^{\prime}}$ induces the map $\varphi_{E, \Xi, \Lambda}$. Obviously, it suffices to prove this observation for $\Xi=\Xi^{\prime}$ and $\Lambda=\Lambda^{\prime}$. In this case, we put, for $\lambda \in \mathfrak{a}_{\mathbb{C}}^{*}$,

$$
\pi_{\lambda}:=\oplus_{\xi \in \Xi} \pi_{P_{0}, \xi, \lambda} .
$$

Then $\pi$ is a holomorphic family of admissible smooth representations of $G$ over $\mathfrak{a}_{\mathbb{C}}^{*}$, so that the functor $X_{\pi}: E \mapsto \pi^{(E)}$ has the exactness properties of Lemma 2.44. Hence, $\pi_{\lambda}^{(E)}$ is a subquotient of $\pi_{\lambda}^{\left(E^{\prime}\right)}$, and $\varphi_{E, \Xi, \lambda}$ is induced by $\varphi_{E^{\prime}, \Xi, \lambda}$, for all $\lambda \in \Lambda$. The result now follows by taking the direct sum over $\lambda \in \Lambda$.

Proposition 4.10. Let $\varphi \in \mathcal{F}_{P_{0}}$. Then the following conditions are equivalent:

(a) $\varphi \in \mathcal{F}_{P_{0}}\left(\mathcal{D}_{P_{0}}\right)$;

(b) for every finite dimensional $\mathcal{O}_{0}$-module $E$ and every pair of finite sets $\Xi \subset M^{\wedge}$ and $\Lambda \subset \mathfrak{a}_{\mathbb{C}}^{*}$, the endomorphism $\varphi_{E, \Xi, \Lambda}$ belongs to $\operatorname{End}\left(\pi_{E, \Xi, \Lambda}\right)^{\#}$.

Proof. First assume (b). Let $\delta=\left(\delta_{1}, \ldots, \delta_{N}\right)$ be a sequence of data in $\mathcal{D}_{P_{0}}$. Then for (a) it suffices to show that

$$
\varphi_{\delta} \in \operatorname{End}\left(V_{\pi_{\delta}}\right)^{\#},
$$

in view of Lemma 4.4.

We note that, with $\delta_{j}=\left(P_{0}, \xi_{j}, \lambda_{j}, \eta_{j}\right)$,

$$
\pi_{\delta}=\oplus_{j=1}^{n} \pi_{P_{0}, \xi_{j}, \lambda_{j}}^{\left(E_{\eta_{j}}\right)} .
$$

Let $E$ be the direct sum of the modules $E_{\eta_{j}}$, and put $\Xi:=\left\{\xi_{1}, \ldots, \xi_{N}\right\}$ and $\Lambda:=\left\{\lambda_{1}, \ldots, \lambda_{N}\right\}$. For each $1 \leq j \leq N$ and $\lambda \in \mathfrak{a}_{\mathbb{C}}^{*}$, let $\left(\pi_{j}\right)_{\lambda}:=\pi_{P_{0}, \xi_{j}, \lambda}$. Then $\pi_{j}$ is a holomorphic family of admissible smooth representations of $G$ over $\mathfrak{a}_{\mathbb{C}}^{*}$, so that Lemma 2.44 applies to the functors $X_{\pi_{j}}: E \mapsto \pi_{j}^{(E)}$. It follows that

$$
\pi_{\delta_{j}} \preceq \pi_{j \lambda_{j}}^{(E)}, \quad \text { and } \quad \varphi_{\delta_{j}}=\varphi_{P_{0}, \xi_{j}, \lambda_{j}}^{(E)} \mid V_{\delta_{j}}
$$

in a natural fashion (here $\rho \preceq \pi$ indicates that $\rho$ is a subrepresentation of $\pi)$. This in turn implies that

$$
\pi_{\delta} \preceq \pi_{E, \Xi, \Lambda}, \quad \text { and } \quad \varphi_{\delta}=\left.\varphi_{E, \Xi, \Lambda}\right|_{V_{\delta}} .
$$

Hence (4.8) follows.

Conversely, assume (a). Let $E, \Xi, \Lambda$ be as stated in (b). First we consider the case that $E$ is of the form $E_{\eta}$, with $\eta$ a finite sequence in $\mathfrak{a}_{\mathbb{C}}^{*}$. Then

$$
\pi_{E_{\eta}, \Xi, \Lambda}=\oplus_{\xi \in \Xi, \lambda \in \Lambda} \quad \pi_{P_{0}, \xi, \lambda}^{(\eta)}
$$

and it follows from (a) that $\varphi_{E_{\eta}, \Xi, \Lambda}$ belongs to $\operatorname{End}\left(\pi_{E_{\eta}, \Xi, \Lambda}\right)^{\#}$. In view of Lemma 2.44, this implies that $\varphi_{E, \Xi, \Lambda}$ belongs to $\operatorname{End}\left(\pi_{E, \Xi, \Lambda}\right)^{\#}$ for $E$ a direct 
sum of copies of $E_{\eta}$. Finally, let $E$ be arbitrary. Then by Proposition 2.31 the module $E$ is a subquotient of $E_{\eta}^{N}$ for a suitable finite sequence $\eta$ and a suitable $N \in \mathbb{N}$. This implies, again by Lemma 2.44, that $\pi_{E, \Xi, \Lambda}$ is a subquotient of $\pi_{E_{\eta}^{N}, \Xi, \Lambda}$ and that $\varphi_{E, \Xi, \Lambda}$ is induced by $\varphi_{E_{\eta}^{N}, \Xi, \Lambda}$. From this and Lemma 4.9 it follows that $\varphi_{E, \Xi, \Lambda}$ belongs to $\operatorname{End}\left(\pi_{E, \Xi, \Lambda}\right)^{\#}$. Hence (b).

\section{Conditions in terms of the Hecke algebra}

Let $k$ be a field and $A$ a $k$-algebra with an approximate identity $\left(\alpha_{j}\right)_{j \in J}$. This means that

(a) $J$ is a partially ordered set;

(b) for all $j_{1}, j_{2}$ in $J$ with $j_{1} \leq j_{2}$ we have $\alpha_{j_{1}} \alpha_{j_{2}}=\alpha_{j_{2}} \alpha_{j_{1}}=\alpha_{j_{1}}$;

(c) for every $a \in A$ there exists a $j \in J$ such that $\alpha_{j} a=a \alpha_{j}=a$.

The Hecke algebra $\mathbb{H}(G, K)$ is an example of such an algebra (see [8, Chap. I, $\S 6])$. Indeed, let $\left(\vartheta_{j}\right)_{j \in \mathbb{N}}$ be an increasing sequence of finite subsets of $K^{\wedge}$, whose union is $K^{\wedge}$. Then

$$
\alpha_{j}:=\sum_{\delta \in \vartheta_{j}} \operatorname{dim}(\delta) \chi_{\delta^{\vee}}, \quad j \in \mathbb{N},
$$

defines an approximate identity in $\mathbb{H}(G, K)$.

We denote the opposite algebra of $A$ by $A^{o p p}$. It is readily seen that the elements $\alpha_{j} \otimes \alpha_{j}$ form an approximate identity for the algebra $A \otimes A^{o p p}$, so that this algebra is approximately unital.

For $j \in J$, let $A_{j}$ be the set of $a \in A$ with $\alpha_{j} a=a \alpha_{j}=a$. Then it is readily seen that $A_{j}$ is a subalgebra of $A$. Moreover, (b) implies that $A_{j_{1}} \subset A_{j_{2}}$ whenever $j_{1} \leq j_{2}$, and (c) implies that $A$ is the union of the subalgebras $A_{j}$.

5.1. Some general facts on approximately unital $A$-modules. Let $V$ be a left (or right) $A$-module (in particular, $V$ is a $k$-linear space). We say that $V$ is approximately unital if, for every $v \in V$, there exists a $j \in J$ such that $\alpha_{j} \cdot v=v$.

For $j \in J$, let

$$
V_{j}:=\left\{\alpha_{j} \cdot v \mid v \in V\right\} .
$$

Then $V_{j_{1}} \subset V_{j_{2}}$ whenever $j_{1} \leq j_{2}$. We note that $V$ is approximately unital if and only if $\cup_{j \in J} V_{j}=V$.

Let $\operatorname{End}(V)$ denote the algebra of $k$-linear endomorphisms of $V$ and let

$$
\pi: A \longrightarrow \operatorname{End}(V)
$$

denote the canonical algebra homomorphism. Then $V_{j}$ is the image of $\pi\left(\alpha_{j}\right)$. We note that $V_{j}$ is invariant under the action of $A_{j}$, turning this space into a left $A_{j}$-module. Let $V^{j}$ denote the kernel of $\pi\left(\alpha_{j}\right)$. Then since $\alpha_{j}$ is an idempotent, it follows that

$$
V=V^{j} \oplus V_{j} .
$$

For any $A$-module $V$, the submodule $V_{a u}:=A \cdot V$ is approximately unital, and it is in fact the maximal submodule with this property. Note that

$$
V_{a u}=\cup_{j \in J} V_{j} .
$$


We now assume that $V$ is an approximately unital $A$-module. The $k$ linear space $\operatorname{Hom}_{k}(V, k)$ has a natural $A^{o p p}$-module structure. Accordingly, we define

$$
V^{\vee}:=\left(\operatorname{Hom}_{k}(V, k)\right)_{a u} .
$$

We denote by $\operatorname{End}(V)_{0}$ the image of the natural linear map $V \otimes V^{\vee} \rightarrow$ $\operatorname{End}(V)$ induced by $v \otimes v^{\prime} \mapsto\left(u \mapsto v^{\prime}(u) v\right)$.

We will say that an $A$-module $V$ is admissible if $V_{j}$ is finite dimensional for every $j \in J$.

Lemma 5.1. Let $V$ be an admissible approximately unital $A$-module. Then, as $A \otimes A^{\text {opp }}$-modules,

$$
\operatorname{End}(V)_{0}=\operatorname{End}(V)_{a u} .
$$

Proof. It is readily seen that $\operatorname{End}(V)_{0}$ is an $A \otimes A^{o p p}$-submodule of $\operatorname{End}(V)$ which is approximately unital. This implies that $\operatorname{End}(V)_{0} \subset \operatorname{End}(V)_{a u}$.

Conversely, let $j \in J$. We consider the inclusion map $\iota_{j}: V_{j} \rightarrow V$ and the epimorphism $p_{j}: V \rightarrow V_{j}$ determined by $\iota_{j} \circ p_{j}=\pi\left(\alpha_{j}\right)$. These maps induce linear maps $S_{j}: \operatorname{End}(V) \rightarrow \operatorname{End}\left(V_{j}\right)$ and $T_{j}: \operatorname{End}\left(V_{j}\right) \rightarrow \operatorname{End}(V)$ given by

$$
S_{j}: f \mapsto p_{j} \circ f \circ \iota_{j} \quad \text { and } \quad T_{j}: g \mapsto \iota_{j} \circ g \circ p_{j} .
$$

It is readily checked that

$$
S_{j} \circ T_{j}=\operatorname{id}_{\operatorname{End}\left(V_{j}\right)}, \quad T_{j} \circ S_{j}(f)=\pi\left(\alpha_{j}\right) \circ f \circ \pi\left(\alpha_{j}\right)=\pi_{\operatorname{End}(V)}\left(\alpha_{j}\right) f .
$$

Hence, $T_{j}$ is an injective linear map $\operatorname{End}\left(V_{j}\right) \rightarrow \operatorname{End}(V)$ with image $\operatorname{End}(V)_{j}$. One now readily checks that the following diagram commutes:

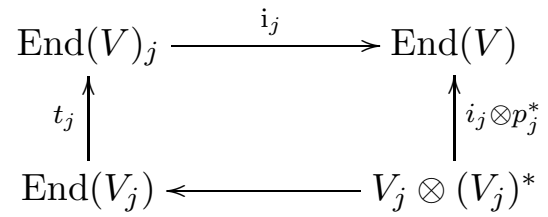

Here $\mathrm{i}_{j}$ is the inclusion map, and $t_{j}$ is the map uniquely determined by $\mathrm{i}_{j} \circ t_{j}=T_{j}$. The map at the bottom is the canonical inclusion.

We now observe that the map at the bottom is a linear isomorphism by admissibility of $V$. Moreover, $p_{j}^{*}$ maps $\left(V_{j}\right)^{*}$ into $\left(V^{*}\right)_{j} \subset V^{\vee}$, and we infer that $\operatorname{End}(V)_{j} \subset \operatorname{End}(V)_{0}$. Finally, by taking the union over all $j$, we conclude that $\operatorname{End}(V)_{a u} \subset \operatorname{End}(V)_{0}$.

\subsection{A double commutant theorem.}

Definition 5.2. Let $(\pi, V)$ be an approximately unital $A$-module. We define $\operatorname{End}(\pi)^{\#}$ to be the space of $\varphi \in \operatorname{End}(V)_{0}$ such that for every $n \in \mathbb{N} \backslash\{0\}$ and every $A$-submodule $W \subset V^{\times n}$ we have $\varphi^{\times n}(W) \subset W$.

Lemma 5.3. Let $(\pi, V)$ be an admissible approximately unital A-module. Then

$$
\pi(A)=\operatorname{End}(\pi)^{\#} .
$$

Proof. Since $\pi(A)$ is an approximately unital $A \otimes A^{\text {opp }}$-submodule of $\operatorname{End}(V)$, it is contained in $\operatorname{End}(V)_{a u}=\operatorname{End}(V)_{0}$. Moreover, if $a \in A$, then for every $n \in \mathbb{N} \backslash\{0\}$ and every invariant subspace $W$ of $V^{\times n}$ we have $\pi(a)^{\times n}(W) \subset W$. Hence, $\pi(A) \subset \operatorname{End}(\pi)^{\#}$. 
We now turn to the converse inclusion. By definition $\mathcal{E}:=\operatorname{End}(\pi)^{\#}$ is a subspace of $\operatorname{End}(V)_{0}=\operatorname{End}(V)_{a u}$. Moreover $\mathcal{E}$ is $A \otimes A^{o p p}$-invariant, hence an approximately unital $A \otimes A^{o p p}$-module. Fix $\varphi \in \mathcal{E}$. Then it follows that there exists a $j \in J$ such that $\left(\alpha_{j} \otimes \alpha_{j}\right) \cdot \varphi=\varphi$.

By admissibility, the space $V_{j}$ has a finite basis $u_{1}, \ldots, u_{n}$ over $k$. Let $W$ be the $A$-submodule of $V^{\times n}$ generated by $\left(u_{1}, \ldots, u_{n}\right)$. Then by definition of $\operatorname{End}(\pi)^{\#}$, the space $W$ is $\varphi^{\times n}$-invariant. This implies the existence of an element $a \in A$ such that $\varphi\left(u_{k}\right)=\pi(a) u_{k}$ for all $1 \leq k \leq n$. Hence $\varphi=\pi(a)$ on $V_{j}$. It follows that $\varphi=\pi\left(a \alpha_{j}\right)$ on $V_{j}$. On the other hand, both $\varphi=\left(\alpha_{j} \otimes \alpha_{j}\right) \cdot \varphi=\pi\left(\alpha_{j}\right) \circ \varphi \circ \pi\left(\alpha_{j}\right)$ and $\pi\left(a \alpha_{j}\right)$ vanish on $V^{j}$. In view of (5.1) this implies that $\varphi=\pi\left(a \alpha_{j}\right)$ on $V$. Hence $\varphi=\pi\left(a \alpha_{j}\right) \in \pi(A)$ and the proof is complete.

5.3. Application to Harish-Chandra modules. Every admissible $(\mathfrak{g}, K)$ module $V$ is a module for the Hecke algebra $\mathbb{H}(G, K)$ in a natural way, and as such it is admissible and approximately unital. This assignment of an $\mathbb{H}(G, K)$-module to an admissible $(\mathfrak{g}, K)$-module defines a functor which establishes an isomorphism of categories, from the category of admissible $(\mathfrak{g}, K)$-modules onto the category of admissible approximately unital $\mathbb{H}(G, K)$-modules (see [8, I, $\S 6$, Theorem 1.117]).

Accordingly, if $V$ is an admissible $(\mathfrak{g}, K)$-module, then the associated algebra $\operatorname{End}(\pi)^{\#}$ consists of all $K \times K$-finite endomorphisms $\varphi$ of $V$ with the property that, for every positive integer $n$, the map $\varphi^{\times n}$ preserves all $(\mathfrak{g}, K)$-invariant subspaces of $V^{\times n}$. In particular, the present notation is compatible with the notation introduced earlier in Definition 4.8.

Corollary 5.4. Let $(\pi, V)$ be an admissible $(\mathfrak{g}, K)$-module. Then

$$
\pi(\mathbb{H}(G, K))=\operatorname{End}(\pi)^{\#} .
$$

Proof. This follows from Lemma 5.3 .

5.4. Proof of the main theorem. For $P \in \mathcal{P}(A)$ we define $\operatorname{PW}_{P}^{\text {pre }}(G, K)$ to be the subspace of $\mathcal{F}_{P}$ (see (4.1)), consisting of all functions $\varphi \in \mathcal{F}_{P}$ such that there exists an $R>0$ and for all $n \in \mathbb{N}$ a constant $C_{n}>0$ such that the estimate (0.2) holds for all $\xi \in M_{P, d s}^{\wedge}$ and all $\lambda \in \mathfrak{a}_{P \mathbb{C}}^{*}$. Furthermore, we define

$$
\mathrm{PW}^{\mathrm{pre}}(G, K)=\oplus_{P \in \mathcal{P}(A)} \mathrm{PW}_{P}^{\mathrm{pre}}(G, K) .
$$

Then $\mathrm{PW}^{\text {pre }}(G, K)$ is a subspace of $\mathcal{F}$. We recall that $P_{0} \in \mathcal{P}(A)$ is a fixed minimal parabolic subgroup of $G$.

\section{Definition 5.5.}

(a) The Delorme Paley-Wiener space $\mathrm{PW}^{D}(G, K)$ is defined to be the intersection $\mathrm{PW}^{\mathrm{pre}}(G, K) \cap \mathcal{F}(\mathcal{D})$.

(b) The restricted Delorme Paley-Wiener space $\mathrm{PW}_{P_{0}}^{D}(G, K)$ is defined to be the intersection $\mathrm{PW}_{P_{0}}^{\mathrm{pre}}(G, K) \cap \mathcal{F}_{P_{0}}\left(\mathcal{D}_{P_{0}}\right)$.

Theorem 5.6. The natural projection pr: $\mathcal{F} \rightarrow \mathcal{F}_{P_{0}}$ restricts to an isomorphism of $\mathrm{PW}^{D}(G, K)$ onto $\mathrm{PW}_{P_{0}}^{D}(G, K)$. 
Proof. It is clear that pr maps $\mathrm{PW}^{\mathrm{pre}}(G, K)$ into $\mathrm{PW}_{P_{0}}^{\mathrm{pre}}(G, K)$. Combining this with Proposition 4.5, we see that $\operatorname{pr}_{\mathcal{D}}$ maps $\mathrm{PW}^{D}(G, K)$ injectively into $\mathrm{PW}_{P_{0}}^{D}(G, K)$.

Let $\tau: \mathcal{F}_{P_{0}} \rightarrow \mathcal{F}$ be defined as in the proof of Proposition 4.5. Then it is readily checked that $\tau$ maps $\mathrm{PW}_{P_{0}}^{\mathrm{pre}}(G, K)$ into $\mathrm{PW}^{\mathrm{pre}}(G, K)$. This implies that $\tau_{\mathcal{D}}$ maps $\mathrm{PW}_{P_{0}}^{D}(G, K)$ into $\operatorname{PW}^{D}(G, K)$. As $\operatorname{pr}_{\mathcal{D}} \circ \tau_{\mathcal{D}}=$ id on $\mathrm{PW}_{P_{0}}^{D}(G, K)$, it follows that $\operatorname{pr}_{\mathcal{D}}$ maps the space $\mathrm{PW}^{D}(G, K)$ surjectively onto $\mathrm{PW}_{P_{0}}^{D}(G, K)$.

Definition 5.7. We define $\mathrm{PW}^{H}(G, K)$ to be the space of functions $\varphi \in$ $\mathrm{PW}_{P_{0}}^{\mathrm{pre}}(G, K)$, such that for every finite dimensional $\mathcal{O}_{0}$-module $E$, and every pair of finite sets $\Xi \subset M^{\wedge}, \Lambda \subset \mathfrak{a}_{\mathbb{C}}^{*}$, we have

$$
\varphi_{E, \Xi, \Lambda} \in \pi_{E, \Xi, \Lambda}(\mathbb{H}(G, K)) .
$$

Theorem 5.8. The space $\mathrm{PW}_{P_{0}}^{D}(G, K)$ equals $\mathrm{PW}^{H}(G, K)$.

Proof. Both spaces are subspaces of $\mathrm{PW}_{P_{0}}^{\text {pre }}(G, K)$. Let $\varphi$ be an element of the latter space. Then $\varphi \in \mathrm{PW}_{P_{0}}^{D}(G, K)$ is equivalent to $\varphi \in \mathcal{F}_{P_{0}}\left(\mathcal{D}_{P_{0}}\right)$, which in turn is equivalent to condition (b) of Proposition 4.10, In view of Corollary 5.4, the latter condition is equivalent to $\varphi \in \mathrm{PW}^{H}(G, K)$.

Definition 5.9. We define the Arthur Paley-Wiener space $\mathrm{PW}^{A}(G, K)$ to be the space of functions $\varphi \in \mathrm{PW}_{P_{0}}^{\mathrm{pre}}(G, K)$ such that $\varphi$ satisfies the ArthurCampoli relations (see Definition 3.2).

Theorem 5.10. The space $\mathrm{PW}^{H}(G, K)$ equals $\mathrm{PW}^{A}(G, K)$.

Proof. Both are subspaces of $\mathrm{PW}_{P_{0}}^{\text {pre }}(G, K)$. Let $\varphi$ be a function in the latter space. Then by Proposition 3.3 the assertion $\varphi \in \mathrm{PW}^{A}(G, K)$ is equivalent to the assertion that for every finite dimensional $\mathcal{O}_{0}$-module, and every pair of finite subsets $\Xi \subset M^{\wedge}, \Lambda \subset \mathfrak{a}_{\mathbb{C}}^{*}$, we have

$$
\varphi_{E, \Xi, \Lambda} \in\left(\pi_{E, \Xi, \Lambda}\left(C_{c}^{\infty}(G, K)\right)^{\perp}\right)^{\perp} .
$$

As every function from $\mathrm{PW}_{P_{0}}^{\mathrm{pre}}(G, K)$ has values in

$$
\oplus_{\xi \in M^{\wedge}} \operatorname{End}\left(C^{\infty}(K: \xi)\right)_{\theta \theta}
$$

for some finite subset $\theta \subset K^{\wedge}$, it follows by admissibility that (5.2) is equivalent to

$$
\varphi_{E, \Xi, \Lambda} \in \pi_{E, \Xi, \Lambda}\left(C_{c}^{\infty}(G, K)\right) .
$$

By Proposition 1.2 this in turn is equivalent to

$$
\varphi_{E, \Xi, \Lambda} \in \pi_{E, \Xi, \Lambda}(\mathbb{H}(G, K)) .
$$

If follows that $\varphi \in \mathrm{PW}^{A}(G, K) \Longleftrightarrow \varphi \in \mathrm{PW}^{H}(G, K)$.

We now come to the main result of our paper.

Theorem 5.11. The map pr: $\varphi \mapsto \varphi_{P_{0}}$ defines a linear isomorphism from $\mathrm{PW}^{D}(G, K)$ onto $\mathrm{PW}^{A}(G, K)$.

Proof. This follows from combining Theorems 5.6, 5.8 and 5.10 . 
5.5. Another useful characterization of the Paley-Wiener space. In this subsection we will obtain another useful characterization of the PaleyWiener space $\mathrm{PW}^{A}(G, K)$. This will allow us to derive the Paley-Wiener theorem due to Helgason [7] and Gangolli [6] from Arthur's Paley-Wiener theorem.

We define the Fourier transform $\mathfrak{F}: C_{c}^{\infty}(G, K)_{K \times K} \rightarrow \mathcal{O}\left(\mathfrak{a}_{\mathbb{C}}^{*}\right) \otimes \mathcal{S}_{P_{0}}$ by $\mathfrak{F}(f)_{\xi}(\lambda)=\widehat{f}\left(P_{0}, \xi, \lambda\right)$, see (3.3). From this definition it is immediate that $\mathfrak{F}$ is a $K \times K$-equivariant linear map.

In terms of this Fourier transform, Arthur's Paley-Wiener theorem may be stated as follows (see [1] and [2]).

Theorem 5.12 (Arthur's Paley-Wiener theorem). The map $\mathfrak{F}$ is a $K \times K$ equivariant linear isomorphism from $C_{c}^{\infty}(G, K)_{K \times K}$ onto $\mathrm{PW}^{A}(G, K)$.

By Theorem 5.10 the Paley-Wiener space $\mathrm{PW}^{A}(G, K)$ equals the space $\mathrm{PW}^{H}(G, K)$ introduced in Definition 5.7. We shall now give another characterization of that space.

We use the notation PW(a) for the (Euclidean) Paley-Wiener space associated with $\mathfrak{a}$, i.e., $\mathrm{PW}(\mathfrak{a})$ is the image of the classical Fourier transform $C_{c}^{\infty}(\mathfrak{a}) \rightarrow \mathcal{O}\left(\mathfrak{a}_{\mathbb{C}}^{*}\right)$. Then the space $\mathrm{PW}_{P_{0}}^{\text {pre }}(G, K)$, introduced in the beginning of Subsection 5.4, equals $\mathrm{PW}(\mathfrak{a}) \otimes \mathcal{S}\left(P_{0}\right)$.

Proposition 5.13. The space $\mathrm{PW}^{H}(G, K)$ is equal to the space of all functions $\varphi \in \mathrm{PW}(\mathfrak{a}) \otimes \mathcal{S}\left(P_{0}\right)$ with the following property.

For each finite number of triples $\left(u_{j}, \xi_{j}, \lambda_{j}\right) \in S\left(\mathfrak{a}^{*}\right) \times M^{\wedge} \times \mathfrak{a}_{\mathbb{C}}^{*}, 1 \leq j \leq n$, there exists an element $h \in \mathbb{H}(G, K)$ such that

$$
\varphi\left(\xi_{j}, \lambda_{j} ; u_{j}\right)=\pi_{P_{0}, \xi_{j}, \lambda_{j} ; u_{j}}(h) \quad \text { for all } \quad 1 \leq j \leq n .
$$

Proof. We first assume that $\varphi \in \mathrm{PW}^{H}(G, K)$. Then $\varphi \in \mathrm{PW}(A) \otimes \mathcal{S}\left(P_{0}\right)$. Let a finite number of triples $\left(u_{j}, \xi_{j}, \lambda_{j}\right)$ be given. For each $u_{j}$ there exists a finite dimensional $\mathcal{O}_{0}$-module $E_{j}$, and a linear functional $\eta_{j} \in \operatorname{End}\left(E_{j}\right)^{*}$ such that $\eta_{j} \circ f^{\left(E_{j}\right)}=f\left(\lambda_{j} ; u_{j}\right)$, for all $f \in \mathcal{O}\left(\mathfrak{a}_{\mathbb{C}}^{*}\right)$; see Lemma 2.11. Put $E=\oplus E_{j}$, and let $\mathrm{pr}_{j}: E \rightarrow E_{j}$ denote the associated projection maps. There exists an element $h \in \mathbb{H}(G, K)$ such that $\varphi_{E, \Xi, \Lambda}=\pi_{E, \Xi, \Lambda}(h)$. This implies that $\varphi_{E, \xi_{j}, \lambda_{j}}=\pi_{E, \xi_{j}, \lambda_{j}}(h)$. for each $j$. By application of $\eta_{j} \circ \operatorname{pr}_{j} \otimes I$ to the latter expression it follows that $\varphi\left(\xi_{j}, \lambda_{j} ; u_{j}\right)=\pi_{P_{0}, \xi_{j}, \lambda_{j} ; u_{j}}(h)$, for all $j$. This proves that $\mathrm{PW}^{H}(G, K)$ is included in the space described.

To obtain the other inclusion, let $\varphi \in \mathrm{PW}(\mathfrak{a}) \otimes \mathcal{S}\left(P_{0}\right)$ satisfy the conditions of the space described. Let $E$ be a finite dimensional $\mathcal{O}_{0}$-module, and let $\Xi \subset M^{\wedge}$ and $\Lambda \subset \mathfrak{a}_{\mathbb{C}}^{*}$ be finite sets. Fix a basis $\left\{\eta^{k}\right\}$ of $\operatorname{End}(E)^{*}$. We may number the elements of $\Lambda$ by $\lambda_{j}$. Then for each $j, k$ there exists a $u_{j}^{k} \in S\left(\mathfrak{a}^{*}\right)$ such that $\eta^{k} f^{(E)}\left(\lambda_{j}\right)=f\left(\lambda_{j} ; u_{j}^{k}\right)$ for all $f \in \mathcal{O}\left(\mathfrak{a}_{\mathbb{C}}^{*}\right)$, see Lemma 2.10. By the assumption on $\varphi$, there exists an element $h \in \mathbb{H}(G, K)$ such that $\varphi\left(\xi, \lambda_{j} ; u_{j}^{k}\right)=\pi_{P_{0}, \xi, \lambda_{j} ; u_{j}^{k}}(h)$ for all $\xi \in \Xi$ and all $j, k$. It follows that

$$
\left(\eta^{k} \otimes 1\right) \circ \varphi_{E, \xi, \lambda_{j}}=\left(\eta^{k} \otimes 1\right) \circ \pi_{E, \xi, \lambda_{j}}(h),
$$

for all $k, j$ and $\xi \in \Xi$. This implies that $\varphi_{E, \xi, \lambda_{j}}=\pi_{E, \xi, \lambda_{j}}(h)$ for all $j$ and all $\xi \in \Xi$. Hence, $\varphi_{E, \Xi, \Lambda}=\pi_{E, \Xi, \Lambda}(h)$, and we conclude that $\varphi \in \mathrm{PW}^{H}(G, K)$. 
Corollary 5.14. The space $\mathrm{PW}^{H}(G, K)_{11}$ of $K \times K$-fixed elements in $\mathrm{PW}^{H}(G, K)$ consists of all $\varphi \in \mathrm{PW}(\mathfrak{a}) \otimes \mathcal{S}\left(P_{0}, 1\right)_{11}$ such that for each finite number of pairs $\left(u_{i}, \lambda_{i}\right) \in S\left(\mathfrak{a}^{*}\right) \times \mathfrak{a}_{\mathbb{C}}^{*}, 1 \leq j \leq n$, there exists an element $h \in \mathbb{H}(G, K)_{11}$ such that

$$
\varphi\left(\lambda_{j} ; u_{j}\right)=\pi_{P_{0}, 1, \lambda_{j} ; u_{j}}(h) \quad \text { for all } \quad 1 \leq j \leq n .
$$

Proof. Since $\mathcal{S}\left(P_{0}\right)_{11}=\mathcal{S}\left(P_{0}, 1\right)_{11}$, this follows from the previous result by projection onto the $K \times K$-type $(1,1)$.

Let $P_{1} \in \operatorname{End}\left(C^{\infty}(K: 1)\right)$ denote the $K$-equivariant projection onto the one-dimensional subspace of $C^{\infty}(M \backslash K)$ consisting of the constant functions. Then we observe that

$$
\mathcal{S}\left(P_{0}, 1\right)_{11} \simeq \operatorname{End}\left(C^{\infty}(K: 1)\right)_{11}=\mathbb{C} P_{1} .
$$

Accordingly, we may may view $\mathrm{PW}^{H}(G, K)_{11}$ as a subspace of $\mathrm{PW}(\mathfrak{a}) \otimes \mathbb{C} P_{1}$.

The inclusion map $\iota: K \rightarrow G$ induces a continuous linear map $\iota^{*}: C^{\infty}(G) \rightarrow$ $C^{\infty}(K)$ by pull-back. The transposed of this map is an injective continuous linear map $\iota_{*}: \mathcal{E}^{\prime}(K) \rightarrow \mathcal{E}^{\prime}(G)$. Accordingly, we shall use this map to view $\mathcal{E}^{\prime}(K)$ as a subspace of $\mathcal{E}^{\prime}(G)$. In particular, the normalized Haar measure $d k$ will be viewed as the element of $\mathbb{H}(G, K)$ given by $d k(\varphi)=\int_{K} \varphi(k) d k$, for $\varphi \in C^{\infty}(G)$. Clearly, $d k \in \mathbb{H}(G, K)_{11}$.

Lemma 5.15. The map $u \mapsto R_{u} d k$ induces a linear isomorphism from $U(\mathfrak{g})^{K} / U(\mathfrak{g})^{K} \cap U(\mathfrak{g})^{\mathfrak{k}}$ onto $\mathbb{H}(G, K)_{11}$.

Proof. We define the linear map $\alpha: U(\mathfrak{g}) \otimes \mathcal{E}^{\prime}(K) \rightarrow \mathcal{E}^{\prime}(G)$ by

$$
\alpha(u \otimes T)=R_{u} T,
$$

where $R$ denotes the right regular representation on $\mathcal{E}^{\prime}(G)$. Then $\alpha$ factors to a linear isomorphism

$$
\bar{\alpha}: U(\mathfrak{g}) \otimes_{U(\mathfrak{k})} \mathcal{E}^{\prime}(K) \rightarrow \mathbb{H}(G, K),
$$

see $[8, \mathrm{I}, \S 6]$. This map intertwines the $K \times K$-actions $(\operatorname{Ad} \otimes R) \times(1 \otimes L)$ and $R \times L$, hence restricts to an isomorphism

$$
\left(U(\mathfrak{g}) \otimes_{U(\mathfrak{k})} \mathcal{E}^{\prime}(K)\right)_{11} \stackrel{\simeq}{\longrightarrow} \mathbb{H}(G, K)_{11}
$$

The space of left $K$-invariants in $\mathcal{E}^{\prime}(K)$ equals $\mathbb{C} d k$. As $d k$ is also right $K$-invariant, we see that

$$
\left(U(\mathfrak{g}) \otimes_{U(\mathfrak{k})} \mathcal{E}^{\prime}(K)\right)_{11} \simeq U(\mathfrak{g})^{K} \otimes_{U(\mathfrak{k})} \mathbb{C} d k \simeq U(\mathfrak{g})^{K} / U(\mathfrak{g})^{K} \cap U(\mathfrak{g}) \mathfrak{k} .
$$

The result now follows.

In the following lemma, $W$ denotes the Weyl group of $\mathfrak{a}$ in $\mathfrak{g}$.

Lemma 5.16. The image of $\mathbb{H}(G, K)_{11}$ under Fourier transform $h \mapsto \widehat{h}$ equals the subspace $P\left(\mathfrak{a}_{\mathbb{C}}^{*}\right)^{W} \otimes \mathbb{C} P_{1}$ of $\mathcal{O}\left(\mathfrak{a}_{\mathbb{C}}^{*}\right) \otimes \mathcal{S}\left(P_{0}\right)$.

Proof. Denote the image by $S$. Then $S$ is contained in $\mathcal{O}\left(\mathfrak{a}_{\mathbb{C}}^{*}\right) \otimes \mathcal{S}\left(P_{0}\right)_{11} \simeq$ $\mathcal{O}\left(\mathfrak{a}_{\mathbb{C}}^{*}\right) \otimes \mathbb{C} P_{1}$. Let $h \in \mathbb{H}(G, K)_{11}$. Then it follows that the Fourier transform 
of $h$ is of the form $\psi \otimes P_{1}$, with $\psi \in \mathcal{O}\left(\mathfrak{a}_{\mathbb{C}}^{*}\right)$. In view of the previous result, $h=R_{u}(d k)$, with $u \in U(\mathfrak{g})^{K}$. Hence, for all $\lambda \in \mathfrak{a}_{\mathbb{C}}^{*}$,

$$
\begin{aligned}
\psi(\lambda) 1_{M \backslash K} & =\left(\psi(\lambda) \otimes P_{1}\right) 1_{M \backslash K} \\
& =\pi_{P_{0}, 1, \lambda}(h) 1_{M \backslash K} \\
& =\pi_{P_{0}, 1, \lambda}(u) 1_{M \backslash K}=\gamma(u, \lambda) 1_{M \backslash K},
\end{aligned}
$$

where $\gamma$ denotes the Harish-Chandra algebra homomorphism $U(\mathfrak{g})^{K} \rightarrow S(\mathfrak{a})$ which has image $S(\mathfrak{a})^{W}=P\left(\mathfrak{a}_{\mathbb{C}}^{*}\right)^{W}$. The result now follows by application of Lemma 5.15

Corollary 5.17. The space $\mathrm{PW}^{H}(G, K)_{11}$ equals $\mathrm{PW}(\mathfrak{a})^{W} \otimes \mathbb{C} P_{1}$.

Proof. Let $\varphi \in \mathrm{PW}^{H}(G, K)_{11}$. Let $\lambda \in \mathfrak{a}_{\mathbb{C}}^{*}$ and $w \in W$. Then by Corollary 5.14 there exists an element $h \in \mathbb{H}(G, K)_{11}$ such that $\varphi(\lambda)=\widehat{h}\left(P_{0}, 1, \lambda\right)$ and $\varphi(w \lambda)=\widehat{h}\left(P_{0}, 1, w \lambda\right)$. As $\widehat{h}\left(P_{0}, 1, \lambda\right)=\widehat{h}\left(P_{0}, 1, w \lambda\right)$ by Lemma 5.16, we see that $\varphi$ is $W$-invariant. Hence, $\varphi \in \mathrm{PW}(\mathfrak{a})^{W} \otimes \mathbb{C} P_{1}$.

Conversely, assume that $\varphi \in \operatorname{PW}(\mathfrak{a})^{W} \otimes \mathbb{C} P_{1}$. Write $\varphi=\psi \otimes P_{1}$, then $\psi$ is a $W$-invariant holomorphic function. Let $\left(u_{j}, \lambda_{j}\right) \in S\left(\mathfrak{a}^{*}\right) \times \mathfrak{a}_{\mathbb{C}}^{*}, 1 \leq j \leq n$. Then there exists an element $p \in P\left(\mathfrak{a}^{*}\right)^{W}$ such that

$$
\psi\left(\lambda_{j} ; u_{j}\right)=p\left(\lambda_{j} ; u_{j}\right) \quad \text { for all } \quad 1 \leq j \leq n .
$$

In view of Lemma 5.16 there exists an element $h \in \mathbb{H}(G, K)_{11}$ such that $\widehat{h}\left(P_{0}, 1, \lambda\right)=p \otimes P_{1}$. Hence,

$$
\varphi\left(\lambda_{j} ; u_{j}\right)=\widehat{h}\left(P_{0}, 1, \lambda_{j} ; u_{j}\right)=\pi_{P_{0}, 1, \lambda_{j} ; u_{j}}(h), \quad(1 \leq j \leq n) .
$$

In view of Corollary 5.14 it follows that $\varphi \in \mathrm{PW}^{H}(G, K)_{11}$.

We now note that the spherical Fourier transform

$$
\mathcal{F}_{11}: C_{c}^{\infty}(G, K)_{11} \rightarrow \mathcal{O}\left(\mathfrak{a}_{\mathbb{C}}^{*}\right)
$$

is given by the formula $\mathcal{F}_{11} f(\lambda) 1_{K / M}=\pi_{P_{0}, 1, \lambda}(f) 1_{K / M}$. This implies that for all $f \in C_{c}^{\infty}(G, K)_{11}$ we have

$$
\mathcal{F}_{11} f(\lambda) \otimes P_{1}=\mathfrak{F} f(\lambda) .
$$

We can now finally deduce the Paley-Wiener theorem of Helgason [7] and Gangolli [6].

Corollary 5.18. $\mathcal{F}_{11}\left(C_{c}^{\infty}(G, K)_{11}\right)=\mathrm{PW}(\mathfrak{a})^{W}$.

Proof. By $K \times K$-equivariance, it follows from Arthur's Paley-Wiener theorem that

$$
\mathcal{F}_{11}\left(C_{c}^{\infty}(G, K)_{11}\right) \otimes \mathbb{C} P_{1}=\mathfrak{F}\left(C_{c}^{\infty}(G, K)_{11}\right)=\mathrm{PW}^{A}(G, K)_{11} .
$$

The latter space equals $\mathrm{PW}^{H}(G, K)_{11}$, by Theorem 5.10 , Now apply Corollary 5.17 . 


\section{Appendix. Some topological Results}

In this appendix, all locally convex spaces will be assumed to be complex and Hausdorff. If $X$ is a locally compact Hausdorff space, and $V$ a quasicomplete locally convex space, then $C(X, V)$, equipped with the topology of uniform convergence on compact subsets, is quasi-complete as well. Let $\Omega$ be an open subset of a finite dimensional complex linear space $\mathfrak{v}_{\mathbb{C}}$. Then $\mathcal{O}(\Omega, V)$, the space of holomorphic functions $\Omega \rightarrow V$, is a closed subspace of $C(\Omega, V)$. Moreover, the map $(f, v) \mapsto(z \mapsto f(z) v)$ induces an embedding of the algebraic tensor product $\mathcal{O}(\Omega) \otimes V$ onto a subspace of $\mathcal{O}(\Omega, V)$. Accordingly, we shall view this tensor product as a subspace. Let $P\left(\mathfrak{v}_{\mathbb{C}}\right)$ denote the space of polynomial functions $\mathfrak{v}_{\mathbb{C}} \rightarrow \mathbb{C}$.

Lemma A.1. The algebraic tensor product $\mathcal{P}\left(\mathfrak{v}_{\mathbb{C}}\right) \otimes V$ is dense in $\mathcal{O}(\Omega, V)$.

In particular, $\mathcal{O}(\Omega) \otimes V$ is a dense subspace of $\mathcal{O}(\Omega, V)$.

Proof. By using partitions of unity, one readily sees that $C(\Omega) \otimes V$ is dense in $C(\Omega, V)$. On the other hand, by application of the Stone-Weierstrass theorem, it follows that $P\left(\mathfrak{v}_{\mathbb{C}}\right)$ is dense in $C(\Omega)$. The lemma now readily follows.

Let $V_{1}, V_{2}$ and $V_{3}$ be quasi-complete locally convex spaces and let

$$
\beta: V_{1} \times V_{2} \rightarrow V_{3}
$$

be a bilinear map. If $X$ is a set, then $\beta$ induces a $\mathbb{C}^{X}$-bilinear map

$$
\begin{aligned}
\beta_{*}: V_{1}^{X} \times V_{2}^{X} & \rightarrow V_{3}^{X} \\
\left(f_{1}, f_{2}\right) & \mapsto\left(x \mapsto \beta\left(f_{1}(x), f_{2}(x)\right)\right) .
\end{aligned}
$$

If $X$ is a locally compact Hausdorff space, we will say that $\beta$ preserves continuity on $X$ if $\beta_{*}$ maps $C\left(X, V_{1}\right) \times C\left(X, V_{2}\right)$ into $C\left(X, V_{3}\right)$. We note that $\beta_{*}: C\left(X, V_{1}\right) \times C\left(X, V_{2}\right) \rightarrow C\left(X, V_{3}\right)$ is $C(X)$-bilinear.

Similarly, if $\Omega$ is an open subset of $\mathfrak{v}_{\mathbb{C}}$, we will say that $\beta$ preserves holomorphy on $\Omega$ if $\beta_{*}$ maps $\mathcal{O}\left(\Omega, V_{1}\right) \times \mathcal{O}\left(\Omega, V_{2}\right)$ into $\mathcal{O}\left(\Omega, V_{3}\right)$. Note that the $\operatorname{map} \beta_{*}$ is $\mathcal{O}(\Omega)$-bilinear.

If $X, Y$ are topological spaces, and $A \subset X$, then a map $f: X \rightarrow Y$ is said to be continuous relative to $A$ if $\left.f\right|_{A}: A \rightarrow Y$ is continuous (with respect to the restriction topology on $A$ ).

Lemma A.2. Assume that $\beta$ is separately continuous and in addition continuous relative to every compact subset of $V_{1} \times V_{2}$. Let $X$ be a locally compact Hausdorff space. Then $\beta$ preserves continuity on $X$. Moreover,

(a) if $V_{1}$ is barrelled, then $\beta_{*}: C\left(X, V_{1}\right) \times C\left(X, V_{2}\right) \rightarrow C\left(X, V_{3}\right)$ is continuous in the first variable;

(b) if $V_{2}$ is barrelled, then $\beta_{*}: C\left(X, V_{1}\right) \times C\left(X, V_{2}\right) \rightarrow C\left(X, V_{3}\right)$ is continuous in the second variable.

Proof. Let $K \subset X$ be compact, and $f_{1} \in C\left(X, V_{1}\right), f_{2} \in C\left(X, V_{2}\right)$. Put $C_{1}:=f_{1}(K), C_{2}:=f_{2}(K)$. Since $\beta$ is continuous relative to $C_{1} \times C_{2}$ and $x \mapsto\left(f_{1}(x), f_{2}(x)\right)$ has continuous restriction to $K$, with values in $C_{1} \times C_{2}$, it follows that $\beta_{*}\left(f_{1}, f_{2}\right)$ is continuous relative to $K$. Since $X$ is locally compact, we see that $\beta_{*}$ has values in $C\left(X, V_{3}\right)$. This establishes the first assertion. 
We now turn to the remaining assertions. By symmetry, it suffices to establish (a). Thus, assume that $V_{1}$ is barrelled and let $f_{2} \in C\left(X, V_{2}\right)$ be fixed. From the first part of the proof we know that $f_{1} \mapsto \beta_{*}\left(f_{1}, f_{2}\right)$ is a linear map from $C\left(X, V_{1}\right)$ to $C\left(X, V_{3}\right)$. To establish its continuity, fix a seminorm $q_{3}$ of $V_{3}$, and a compact subset $K$ of $X$. Put $C_{2}=f_{2}(K)$. Then the family of continuous linear maps $\beta\left(\cdot, v_{2}\right) \in \operatorname{Hom}\left(V_{1}, V_{3}\right)$ is pointwise bounded, for $v_{2} \in C_{2}$. By barrelledness of $V_{1}$ it follows that the family is equicontinuous. Hence, there exists a continuous seminorm $q_{1}$ on $V_{1}$ such that

$$
q_{3}\left(\beta\left(v_{1}, v_{2}\right)\right) \leq q_{1}\left(v_{1}\right), \quad v_{1} \in V_{1}, v_{2} \in C_{2} .
$$

Let $f_{1} \in C\left(X, V_{1}\right)$. Then substituting $f_{1}(x)$ for $v_{1}$ and $f_{2}(x)$ for $v_{2} \quad(x \in K)$ in the above estimate, we find that

$$
\sup _{x \in K} q_{3}\left(\beta_{*}\left(f_{1}, f_{2}\right)(x)\right) \leq \sup _{x \in K} q_{1}\left(f_{1}(x)\right) .
$$

This establishes the continuity.

Lemma A.3. Assume that $V_{1}, V_{2}$ are barrelled, and let $\beta$ be as in Lemma A.2 If $\Omega \subset \mathfrak{v}_{\mathbb{C}}$ is open, then $\beta$ preserves holomorphy on $\Omega$. Moreover, the map $\beta_{*}: \mathcal{O}\left(\Omega, V_{1}\right) \times \mathcal{O}\left(\Omega, V_{2}\right) \rightarrow \mathcal{O}\left(\Omega, V_{3}\right)$ is a separately continuous $\mathcal{O}(\Omega)$ bilinear map.

Proof. It is readily seen that $\beta_{*}$ maps the subspace $\left(\mathcal{O}(\Omega) \otimes V_{1}\right) \times\left(\mathcal{O}(\Omega) \otimes V_{2}\right)$ into the closed subspace $\mathcal{O}\left(\Omega, V_{3}\right)$ of the quasi-complete space $C\left(\Omega, V_{3}\right)$. By continuity of $\beta_{*}$ in the first variable (see Lemma A.2), and by density and closedness, it follows that $\beta_{*}$ maps $\mathcal{O}\left(\Omega, V_{1}\right) \times \mathcal{O}(\Omega) \otimes V_{2}$ into $\mathcal{O}\left(\Omega, V_{3}\right)$. By the same kind of argument applied to the second variable, the result follows.

Examples of a different nature are provided by the composition of maps. If $V, W$ are two locally convex spaces then by $\operatorname{Hom}(V, W)$ we denote the space of continuous complex linear maps $V \rightarrow W$. Unless otherwise specified this space is equipped with the strong operator topology. If $W$ is quasi-complete, and $V$ barrelled, then by application of the principle of uniform boundedness, it follows that $\operatorname{Hom}(V, W)$ is quasi-complete (see, e.g., [3, Ch. III, 27, $\S 4$, no. 2, Cor. 4]).

Assume now that $V_{1}, V_{2}$ and $V_{3}$ are arbitrary locally convex spaces, not necessarily quasi-complete.

Lemma A.4. The map

$$
\beta: \operatorname{Hom}\left(V_{1}, V_{2}\right) \times \operatorname{Hom}\left(V_{2}, V_{3}\right) \rightarrow \operatorname{Hom}\left(V_{1}, V_{3}\right), \quad(A, B) \mapsto B \circ A
$$

is bilinear and separately continuous. If $V_{2}$ is barrelled, then $\beta$ is continuous relative to subsets of the form $\operatorname{Hom}\left(V_{1}, V_{2}\right) \times C$, with $C \subset \operatorname{Hom}\left(V_{2}, V_{3}\right)$ compact.

Proof. As $V_{2}$ is barrelled, every compact subset of $\operatorname{Hom}\left(V_{2}, V_{3}\right)$ is equicontinuous. The result now follows from [3, Ch. III, 33, $\S 5$, no. 5, Prop. 9].

Corollary A.5. Let $V_{1}, V_{2}, V_{3}$ be quasi-complete, and assume $V_{2}$ is barrelled. Let $\beta$ be as in (A.1). Then $\beta$ preserves continuity on $X$, for any locally compact Hausdorff space $X$. 
Proof. This follows from Lemma A.4 combined with Lemma A.2.

Lemma A.6. Let $V_{1}, V_{2}, V_{3}$ be quasi-complete, and assume both $V_{1}$ and $V_{2}$ to be barrelled. Let $\beta$ be the composition map given by (A.1) and let $\Omega \subset \mathfrak{v}_{\mathbb{C}}$ be open. Then $\beta$ preserves holomorphy on $\Omega$.

Proof. Let $A \in \mathcal{O}\left(\Omega, \operatorname{Hom}\left(V_{1}, V_{2}\right)\right)$ and $B \in \mathcal{O}\left(\Omega, \operatorname{Hom}\left(V_{2}, V_{3}\right)\right)$. Then by the previous result it follows that $\beta_{*}(A, B): \mu \mapsto B(\mu) A(\mu)$ is continuous. By barrelledness of $V_{1}$, the function $f:(\mu, v) \mapsto A(\mu) v, \Omega \times V_{1} \rightarrow V_{2}$ is continuous and holomorphic in $\mu$. Similarly, the function $g:\left(\nu, v_{2}\right) \mapsto$ $B(\nu) v_{2}, \Omega \times V_{2} \rightarrow V_{3}$ is continuous and holomorphic in $\nu$. It follows that $h:\left(\mu, \nu, v_{1}\right) \mapsto g\left(\nu, f\left(\mu, v_{1}\right)\right)$ is continuous, and separately holomorphic in $\mu, \nu$. In particular, for fixed $v_{1}$, the map $(\mu, \nu) \mapsto h\left(\mu, \nu, v_{1}\right)$ is holomorphic in each of the two variables. If $\xi \in V_{3}^{\prime}$ then $(\mu, \nu) \mapsto \xi h\left(\mu, \nu, v_{1}\right)$ is continuous and separately holomorphic, hence holomorphic. It follows that $(\mu, \nu) \mapsto h\left(\mu, \nu, v_{1}\right)$ is weakly holomorphic, hence holomorphic. We conclude that $\mu \mapsto h\left(\mu, \mu, v_{1}\right)$ is holomorphic. This implies that the function $\left(\mu, v_{1}\right) \mapsto \beta_{*}(A, B)(\mu) v_{1}$ is continuous on $\Omega \times V_{1}$, holomorphic in $\mu$ and linear in $v$. This in turn implies that $\beta_{*}(A, B)$ is holomorphic as a function with values in $\operatorname{Hom}\left(V_{1}, V_{3}\right)$.

Given an element $u$ of the symmetric algebra $S(\mathfrak{v})$ of $\mathfrak{v}_{\mathbb{C}}$, we will write $\partial_{u}$ for the naturally associated constant coefficient (holomorphic) differential operator on $\mathfrak{v}_{\mathbb{C}}$, see 92.1 . This operator acts on the space of holomorphic functions $\mathcal{O}(\Omega)$, for any open subset $\Omega \subset \mathfrak{v}_{\mathbb{C}}$. We agree to write $f(\cdot ; u)=\partial_{u} f$, for $f \in \mathcal{O}(\Omega)$, see (2.2).

If $V$ is a quasi-complete locally convex space, then $\partial_{u} \otimes \mathrm{id}_{V}$ has a unique extension to a continuous linear endomorphism of $\mathcal{O}(\Omega, V)$. Indeed, uniqueness is obvious from density of $\mathcal{O}(\Omega) \otimes V$ in $\mathcal{O}(\Omega, V)$. Existence follows for instance by application of the Cauchy integral formula.

Let $A$ be a finite dimensional associative algebra. Suppose

$$
M: \mathcal{O}(\Omega) \rightarrow \mathcal{O}(\Omega, A)
$$

is a continuous algebra homomorphism which can be represented by an element from $S(\mathfrak{v}) \otimes A$, i.e., there exist $u_{i} \in S(\mathfrak{v})$ and $a_{i} \in A$ such that

$$
M f=\sum_{i} \partial_{u_{i}} f \cdot a_{i} .
$$

Then it follows from the above that the map $M \otimes \operatorname{id}_{V}$ has a unique extension to a continuous linear map $\mathcal{O}(\Omega, V) \rightarrow \mathcal{O}(\Omega, A \otimes V)$. The extension is denoted by $M \widehat{\otimes} \mathrm{id}_{V}$, or more briefly by $M$ again.

Remark A.7. In the present paper, the following examples are of particular importance. Let $V$ be a quasi-complete locally convex space and let $\mathcal{I}$ be a cofinite ideal in $\mathcal{O}_{0}$. Then $M=J_{\mathcal{I}}$ is of the form (A.2), see (2.4), and defines an algebra homomorphism $\mathcal{O}(\Omega) \rightarrow \mathcal{O}\left(\Omega, \mathcal{O}_{0} / \mathcal{I}\right)$. It follows that $J_{\mathcal{I}} \otimes$ $\operatorname{id}_{V}: \mathcal{O}(\Omega) \otimes V \rightarrow \mathcal{O}\left(\Omega, \mathcal{O}_{0} / \mathcal{I}\right) \otimes V$ has a unique extension to a continuous linear map

$$
J_{\mathcal{I}} \widehat{\otimes} \operatorname{id}_{V}: \mathcal{O}(\Omega, V) \longrightarrow \mathcal{O}\left(\Omega, \mathcal{O}_{0} / \mathcal{I} \otimes V\right),
$$

which will often briefly be denoted by $J_{\mathcal{I}}$ again. 
Similarly, let $E$ be a finite dimensional $\mathcal{O}_{0}$-module. Then $J^{(E)}: \mathcal{O}(\Omega) \rightarrow$ $\mathcal{O}(\Omega, \operatorname{End}(E))$ is an algebra homomorphism of the form (A.2), see Definition 2.7 and Corollary 2.12. Hence, $J^{(E)} \otimes \mathrm{id}_{V}$ has a unique extension to a continuous linear map

$$
J^{(E)} \widehat{\otimes} \operatorname{id}_{V}: \mathcal{O}(\Omega, V) \longrightarrow \mathcal{O}(\Omega, \operatorname{End}(E) \otimes V),
$$

which will often briefly be denoted by $J^{(E)}: f \mapsto f^{(E)}$.

Lemma A.8. Let $V_{1}, V_{2}$ and $V_{3}$ be quasi-complete locally convex Hausdorff spaces, and let $\beta: V_{1} \times V_{2} \rightarrow V_{3}$ be a bilinear form which preserves holomorphy on the open subset $\Omega$ of $\mathfrak{v}_{\mathbb{C}}$. Let $\beta^{\prime}: E_{1} \times E_{2} \rightarrow E_{3}$ be a bilinear form of finite dimensional spaces. Then the bilinear form $\beta^{\prime} \otimes \beta: E_{1} \otimes V_{1} \times E_{2} \otimes V_{2} \rightarrow E_{3} \otimes V_{3}$ preserves holomorphy on $\Omega$ as well.

Proof. This is an easy consequence of the finite dimensionality of the spaces $E_{j}$.

Let $m^{A}$ denote the (continuous) bilinear product map

$$
m^{A}:(a, b) \mapsto a b, \quad A \times A \rightarrow A .
$$

Proposition A.9. Let $V_{1}, V_{2}, V_{3}$ be quasi-complete locally convex Hausdorff spaces, and let $\beta: V_{1} \times V_{2} \rightarrow V_{3}$ be a bilinear form which preserves holomorphy on $\Omega$. Let $A$ be a finite dimensional associative algebra, and let $M: \mathcal{O}(\Omega) \rightarrow$ $\mathcal{O}(\Omega, A)$ be as in (A.2). Then, for all $f_{j} \in \mathcal{O}\left(\Omega, V_{j}\right), j=1,2$,

$$
M \beta_{*}\left(f_{1}, f_{2}\right)=\left(m^{A} \otimes \beta\right)_{*}\left(M f_{1}, M f_{2}\right) .
$$

The following lemma prepares for the proof. Given $\mu \in \Omega$, let $\mathcal{M}_{\mu}$ be the maximal ideal of the algebra $\mathcal{O}(\Omega)$ consisting of the functions vanishing at $\mu$.

Lemma A.10. Let $M$ be as in (A.2). There exists a number $d \in \mathbb{N}$ with the following property. Let $V$ be a quasi-complete locally convex Hausdorff space. Then for every $k \geq d$, the operator $M \widehat{\otimes} \mathrm{id}_{V}$ maps $\mathcal{M}_{\mu}^{k} \mathcal{O}(\Omega, V)$ into $\mathcal{M}_{\mu}^{k-d} \mathcal{O}(\Omega, A \otimes V)$.

Proof. It suffices to show that, for any element $u \in S(\mathfrak{v})$ of order $d$, the differentiation $\partial_{u}$ maps $\mathcal{M}_{\mu}^{k} \mathcal{O}(\Omega, V)$ into $\mathcal{M}_{\mu}^{k-d} \mathcal{O}(\Omega, V)$. Clearly, it suffices to do this for $u=X \in \mathfrak{v}$ and $d=1$. In this case the result follows from the Leibniz rule

$$
\left(\partial_{u} \widehat{\otimes} \operatorname{id}_{V}\right)(\varphi F)=\left(\partial_{u} \varphi\right) F+\varphi\left(\partial_{u} \widehat{\otimes} \operatorname{id}_{V}\right)(F),
$$

for all $\varphi \in \mathcal{O}(\Omega)$ and $F \in \mathcal{O}(\Omega, V)$.

Proof of Proposition A.9. We will first prove the identity for $f_{j} \in \mathcal{O}(\Omega) \otimes V_{j}$, $j=1,2$. Let $m: \mathcal{O}(\Omega) \times \mathcal{O}(\Omega) \rightarrow \mathcal{O}(\Omega)$ denote the multiplication map. Then $\beta_{*}\left(f_{1}, f_{2}\right)=(m \otimes \beta)\left(f_{1}, f_{2}\right) \in \mathcal{O}(\Omega) \otimes V_{3}$. Moreover, since $M: \mathcal{O}(\Omega) \rightarrow$ $\mathcal{O}(\Omega, A)$ is an algebra homomorphism, it follows that

$$
\begin{aligned}
M \beta_{*}\left(f_{1}, f_{2}\right) & =\left(M \otimes \mathrm{id}_{V_{3}}\right) \circ(m \otimes \beta)\left(f_{1}, f_{2}\right) \\
& =\left(m^{A} \otimes \beta\right)_{*}\left(\left[M \otimes \mathrm{id}_{V_{1}}\right] f_{1},\left[M \otimes \mathrm{id}_{V_{2}}\right] f_{2}\right) \\
& =\left(m^{A} \otimes \beta\right)_{*}\left(M f_{1}, M f_{2}\right) .
\end{aligned}
$$

We will now establish the identity for general $f_{j} \in \mathcal{O}\left(\Omega, V_{j}\right)$. Clearly, it suffices to prove the identity of holomorphic functions at a fixed point $\mu$ 
of $\Omega$. Moreover, since the operations $\left(f_{1}, f_{2}\right) \mapsto \beta_{*}\left(f_{1}, f_{2}\right)$ and $M$ commute with restriction, we may assume that $\Omega$ is a polydisk centered at $\mu$. Let $d$ be as in Lemma A.10. Fix $k>d$.

From the power series expansions of $f_{1}, f_{2}$ at the point $\mu$ it follows that there exist polynomials $p_{j} \in P(\mathfrak{v}) \otimes V_{j}$ and holomorphic functions $r_{j} \in$ $\mathcal{M}_{\mu}^{k} \mathcal{O}\left(\Omega, V_{j}\right)$ such that

$$
f_{j}=p_{j}+r_{j}, \quad j=1,2 .
$$

From the $\mathcal{O}(\Omega)$-bilinearity of $\beta_{*}$ it readily follows that

$$
\beta_{*}\left(f_{1}, f_{2}\right)=\beta_{*}\left(p_{1}, p_{2}\right)+R,
$$

with $R \in \mathcal{M}_{\mu}^{k} \mathcal{O}\left(\Omega, V_{3}\right)$. From the $\mathcal{O}(\Omega)$-bilinearity of $\beta_{*}$ combined with Lemma A.10 it follows that

$$
\left(m^{A} \otimes \beta\right)_{*}\left(M f_{1}, M f_{2}\right)=\left(m^{A} \otimes \beta\right)_{*}\left(M p_{1}, M p_{2}\right)+\rho,
$$

with $\rho \in \mathcal{M}_{\mu}^{k-d} \mathcal{O}\left(\Omega, V_{3} \otimes A\right)$. Evaluation at the point $\mu$ gives $\operatorname{ev}_{\mu}(R)=$ $R(\mu)=0$ and $\operatorname{ev}_{\mu}(\rho)=\rho(\mu)=0$. Combining this with (A.3), (A.4) and the first part of the proof applied to $\left(p_{1}, p_{2}\right)$ we find

$$
\begin{aligned}
\operatorname{ev}_{\mu} M \beta_{*}\left(f_{1}, f_{2}\right) & =\operatorname{ev}_{\mu} M \beta_{*}\left(p_{1}, p_{2}\right) \\
& =\operatorname{ev}_{\mu}\left(m^{A} \otimes \beta\right)_{*}\left(M p_{1}, M p_{2}\right) \\
& =\operatorname{ev}_{\mu}\left(m^{A} \otimes \beta\right)_{*}\left(M f_{1}, M f_{2}\right) .
\end{aligned}
$$

This establishes the desired identity at the point $\mu$.

Corollary A.11. Let $V_{1}, V_{2}, V_{3}$ and $\beta, \Omega$ be as Proposition A.9.

(a) For any cofinite ideal $\mathcal{I}$ of $\mathcal{O}_{0}$,

$$
J_{\mathcal{I}} \circ \beta_{*}=\left(m^{\mathcal{O}_{0} / \mathcal{I}} \otimes \beta\right)_{*} \circ\left(J_{\mathcal{I}}, J_{\mathcal{I}}\right)
$$

on $\mathcal{O}\left(\Omega, V_{1}\right) \times \mathcal{O}\left(\Omega, V_{2}\right)$.

(b) For any finite dimensional $\mathcal{O}_{0}$-module $E$,

$$
\begin{aligned}
J^{(E)} & \circ \beta_{*}=\left(m^{\operatorname{End}(E)} \otimes \beta\right)_{*} \circ\left(J^{(E)}, J^{(E)}\right) \\
\text { on } \mathcal{O}\left(\Omega, V_{1}\right) & \times \mathcal{O}\left(\Omega, V_{2}\right) .
\end{aligned}
$$

Proof. In view of Remark A.7, this follows from Proposition A.9.

Corollary A.12. Let $V_{1}, V_{2}, V_{3}$ be quasi-complete locally convex Hausdorff spaces, and assume that $V_{1}, V_{2}$ are barrelled. Let $S \in \mathcal{O}\left(\Omega, \operatorname{Hom}\left(V_{1}, V_{2}\right)\right)$ and $T \in \mathcal{O}\left(\Omega, \operatorname{Hom}\left(V_{2}, V_{3}\right)\right)$. Then the map $T S: \mu \mapsto T(\mu) S(\mu)$ belongs to $\mathcal{O}\left(\Omega, \operatorname{Hom}\left(V_{1}, V_{3}\right)\right)$. Moreover, the following holds.

(a) For any cofinite ideal $\mathcal{I}$ of $\mathcal{O}_{0}$,

$$
J_{\mathcal{I}}(T S)=J_{\mathcal{I}}(T) J_{\mathcal{I}}(S) .
$$

(b) For any finite dimensional $\mathcal{O}_{0}$-module $E$,

$$
(T S)^{(E)}=T^{(E)} S^{(E)} \text {. }
$$

The expression on the right-hand side of (a) should be read as the natural pointwise product of the functions $J_{\mathcal{I}} S \in \mathcal{O}\left(\Omega, \mathcal{O}_{0} / \mathcal{I} \otimes \operatorname{Hom}\left(V_{1}, V_{2}\right)\right)$ and $J_{\mathcal{I}} T \in \mathcal{O}\left(\Omega, \mathcal{O}_{0} / \mathcal{I} \otimes \operatorname{Hom}\left(V_{2}, V_{3}\right)\right)$. 
For (b) we note that $S^{(E)}$ is holomorphic on $\Omega$ with values in $\operatorname{End}(E) \otimes$ $\operatorname{Hom}\left(V_{1}, V_{2}\right) \simeq \operatorname{Hom}\left(E \otimes V_{1}, E \otimes V_{2}\right)$. Likewise, $T^{(E)}$ is a $\operatorname{Hom}\left(E \otimes V_{2}, E \otimes V_{3}\right)$ valued holomorphic function on $\Omega$. Accordingly, the expression on the righthand side of (b) should be read as the pointwise composition.

Proof. This follows from the previous corollary combined with Lemma A.6.

\section{REFERENCES}

1. J. Arthur, A Paley-Wiener theorem for real reductive groups, Acta Math. 150 (1983), no. 1-2, 1-89.

2. E. P. van den Ban and H. Schlichtkrull, Paley-Wiener spaces for real reductive Lie groups, Indag. Math. (N.S.) 16 (2005), no. 3-4, 321-349.

3. N. Bourbaki, Espaces vectoriels topologiques. Chapitres 1 à 5 , new ed., Masson, Paris, 1981, Éléments de mathématique. [Elements of mathematics].

4. W. Casselman, Canonical extensions of Harish-Chandra modules to representations of $G$, Canad. J. Math. 41 (1989), no. 3, 385-438.

5. P. Delorme, Sur le théorème de Paley-Wiener d'Arthur, Ann. of Math. (2) 162 (2005), no. 2, 987-1029.

6. R. Gangolli, On the Plancherel formula and the Paley-Wiener theorem for spherical functions on semisimple Lie groups, Ann. of Math. (2) 93 (1971), 150-165.

7. S. Helgason, Groups and geometric analysis, Mathematical Surveys and Monographs, vol. 83, American Mathematical Society, Providence, RI, 2000, Integral geometry, invariant differential operators, and spherical functions, Corrected reprint of the 1984 original.

8. A. W. Knapp and D. A. Vogan, Jr., Cohomological induction and unitary representations, Princeton Mathematical Series, vol. 45, Princeton University Press, Princeton, NJ, 1995.

9. Nolan R. Wallach, Real reductive groups. I, Pure and Applied Mathematics, vol. 132, Academic Press Inc., Boston, MA, 1988.

10. _ Real reductive groups. II, Pure and Applied Mathematics, vol. 132, Academic Press Inc., Boston, MA, 1992. 